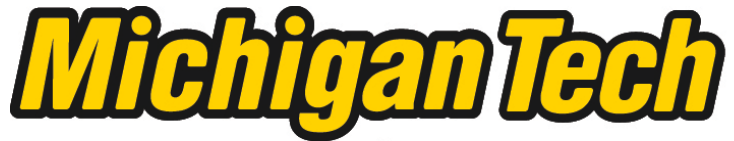 \\ Michigan Technological University Create the Future Digital Commons @ Michigan Tech
}

2014

\section{USE OF AN ELECTRICAL IMPEDANCE TOMOGRAPHY METHOD TO DETECT AND TRACK FRACTURES IN A GELATIN MEDIUM}

Evan G. Lucas

Michigan Technological University

Follow this and additional works at: https://digitalcommons.mtu.edu/etds

Part of the Geophysics and Seismology Commons, Mechanical Engineering Commons, and the Radiology Commons

Copyright 2014 Evan G. Lucas

\section{Recommended Citation}

Lucas, Evan G., "USE OF AN ELECTRICAL IMPEDANCE TOMOGRAPHY METHOD TO DETECT AND TRACK FRACTURES IN A GELATIN MEDIUM", Master's Thesis, Michigan Technological University, 2014.

https://doi.org/10.37099/mtu.dc.etds/742

Follow this and additional works at: https://digitalcommons.mtu.edu/etds

Part of the Geophysics and Seismology Commons, Mechanical Engineering Commons, and the Radiology Commons 


\title{
USE OF AN ELECTRICAL IMPEDANCE TOMOGRAPHY METHOD TO DETECT AND TRACK FRACTURES IN A GELATIN MEDIUM
}

\section{By}

Evan G. Lucas

\begin{abstract}
A THESIS
Submitted in partial fulfillment of the requirements for the degree of MASTER OF SCIENCE

In Mechanical Engineering
\end{abstract}

MICHIGAN TECHNOLOGICAL UNIVERSITY

2014

(C) 2014 Evan G. Lucas 
This thesis has been approved in partial fulfillment of the requirements for the Degree of MASTER OF SCIENCE in Mechanical Engineering.

Department of Mechanical Engineering - Engineering Mechanics

Thesis Advisor: $\quad$ Dr. Jason Blough

Committee Member: Dr. Roger Turpening

Committee Member: Dr. James DeClerck

Department Chair: $\quad$ Dr. William Predebon 


\section{Table of Contents}

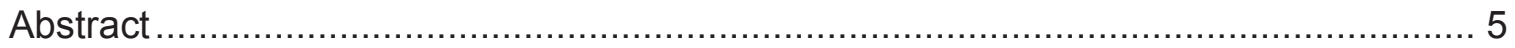

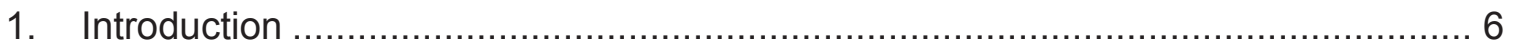

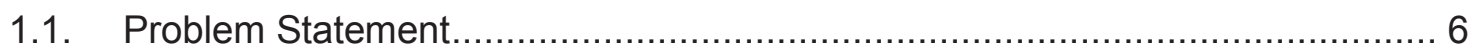

1.2. Overview of Electrical Impedance Tomography .......................................... 6

1.2.1. Applications of Electrical Impedance Tomography ……........................... 7

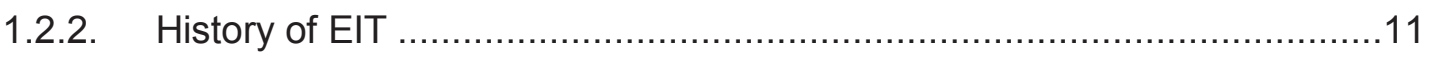

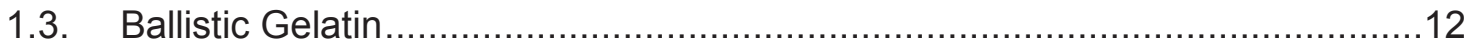

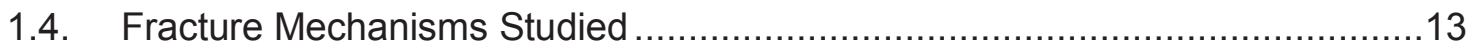

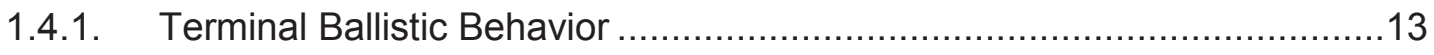

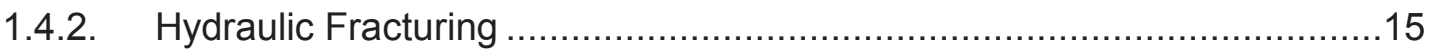

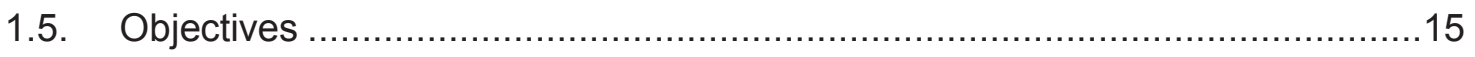

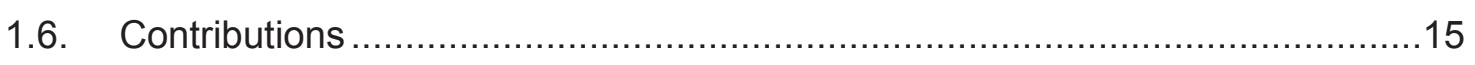

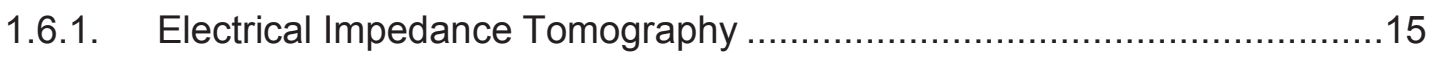

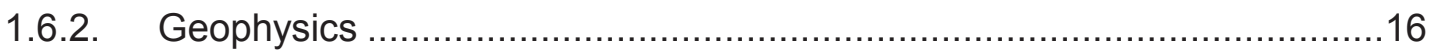

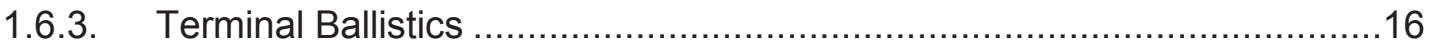

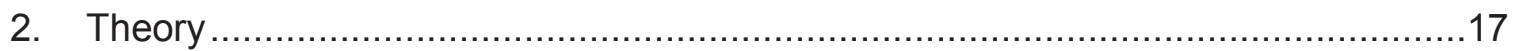

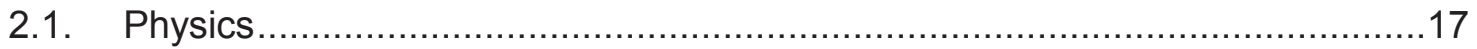

2.1.1. Conceptually understanding Electrical Impedance Tomography...............18

2.1.2. Comparison of EIT to Other Tomographic Imaging Methods ...................24

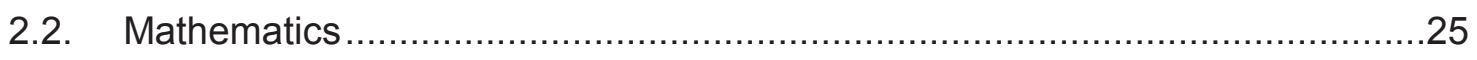

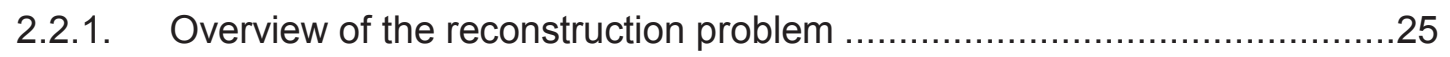

2.2.2. Electrical Impedance Tomography and Diffuse Optical Tomography

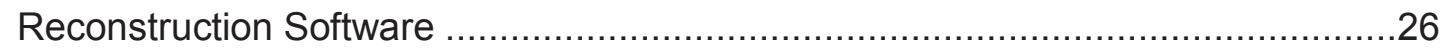

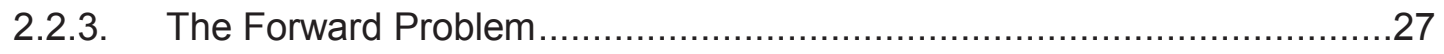

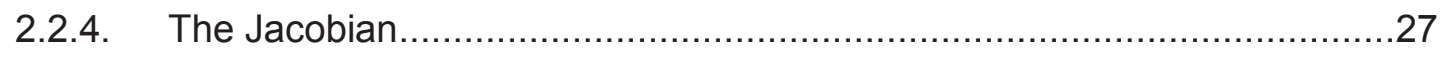

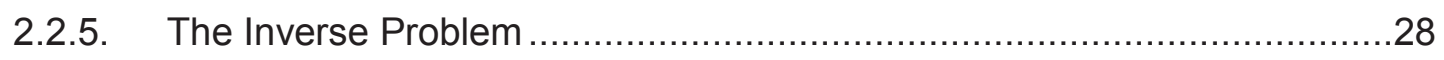

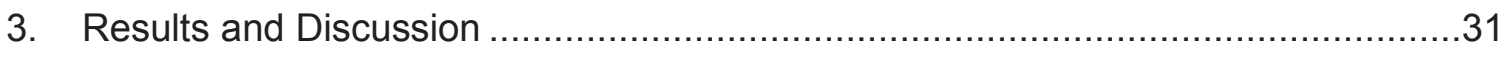

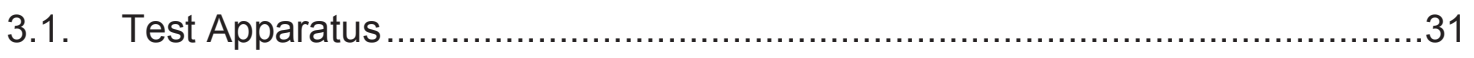

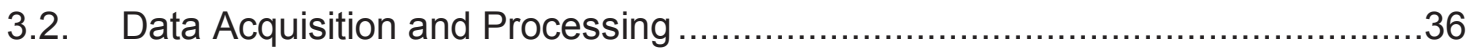

3.2.1. Hardware complications of the system developed for this study ...............36 


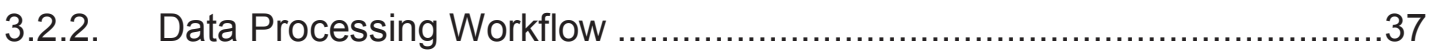

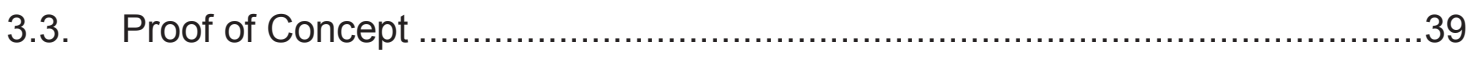

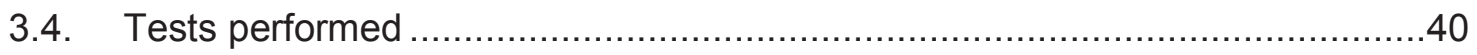

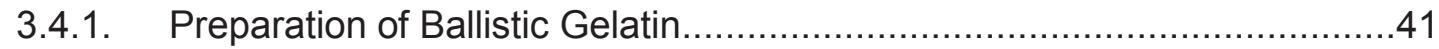

3.4.2. Hydraulic Fracture Imaging …………..........................................

3.4.3. Discussion of Hydraulic Fracture Imaging Results ................................53

3.4.4. Temporary Cavity Fracture Imaging …………....................................54

3.4.5. Discussion of Temporary Cavity Imaging Results...................................64

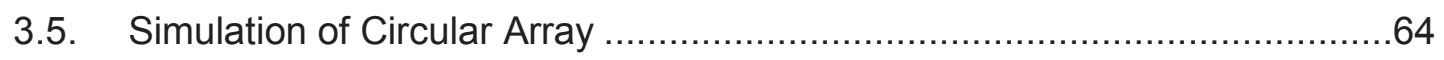

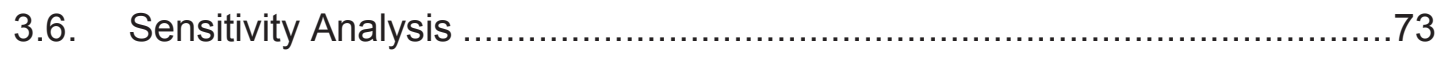

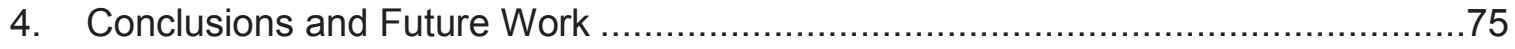

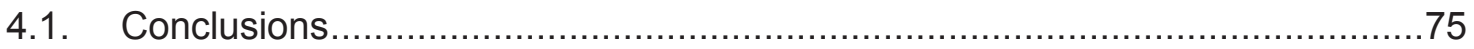

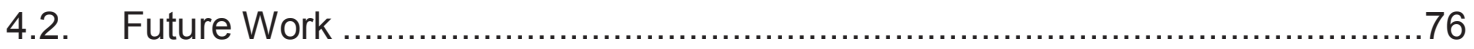

4.2.1. Future Work on Temporary Cavity Imaging ……..................................76

4.2.2. Future Work on Hydraulic Fracturing Imaging ……..............................76

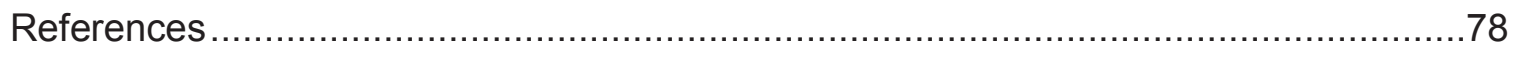

Appendix A: Copyrighted material permission documentation .................................81

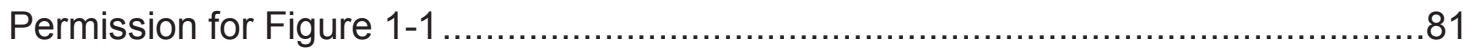

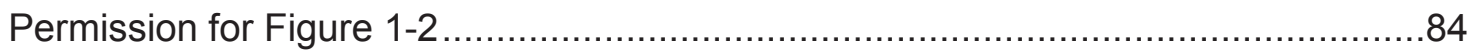

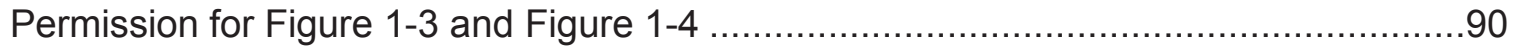

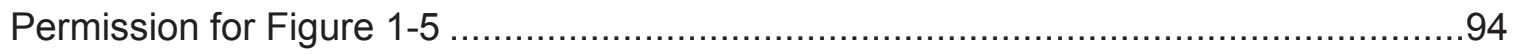

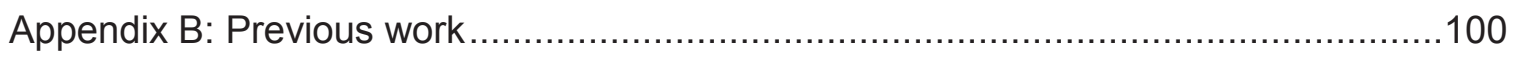




\section{Abstract}

Electrical impedance tomography is applied to the problem of detecting, locating, and tracking fractures in ballistics gelatin. The hardware developed is intended to be physically robust and based on off-the-shelf hardware. Fractures were created in two separate ways: by shooting a .22 caliber bullet into the gelatin and by injecting saline solution into the gelatin. The .22 caliber bullet created an air gap, which was seen as an increase in resistivity. The saline solution created a fluid filled gap, which was seen as a decrease in resistivity. A double linear array was used to take data for each of the fracture mechanisms and a two dimensional cross section was inverted from the data. The results were validated by visually inspecting the samples during the fracture event. It was found that although there were reconstruction errors present, it was possible to reconstruct a representation of the resistive cross section. Simulations were performed to better understand the reconstructed cross-sections and to demonstrate the ability of a ring array, which was not experimentally tested. 


\section{Introduction}

\subsection{Problem Statement}

The goal of this project is to dynamically detect and track fractures in ballistic gelatin. Fractures will be initiated in two ways, by shooting the gelatin with a 22 caliber bullet and by injecting a fluid into the gelatin. The fractures will be detected and analyzed by using an Electrical Impedance Tomography (EIT) system that will be designed to be physically robust and portable.

\subsection{Overview of Electrical Impedance Tomography}

Electrical Impedance Tomography (EIT) is an imaging technique that has been used in the fields of geophysical, industrial, and medical applications. It operates by detecting changes in resistivity, which indicate changes in the physical properties of the system. In brief, a current is applied between two electrodes and the voltage potential is measured between at least two other electrodes[1]. Some advanced systems will inject current into more than two electrodes simultaneously, but the majority of systems today use a single pair for current injection that cycles through pairs of electrodes[2]. By taking multiple measurements with multiple current applications across a system, it is possible to estimate the distribution of resistivities within the system. A more detailed explanation of how EIT works is included in Chapter 2. By taking these measurements repeatedly, resistivity changes with respect to time can be measured. This technique has been applied in one, two, and three dimensional cases[3].

The advantages of EIT are: its nondestructive nature, a relatively low cost to implement and operate, physical robustness and portability of the measurement system, and a high temporal resolution. Compared to other tomography systems, EIT suffers from having an 
inferior spatial resolution and computationally intensive data processing and interpretation[4].

\subsubsection{Applications of Electrical Impedance Tomography}

In geophysics, EIT has found many uses including aquifer exploration, landfill leak detection, agricultural soil testing [5], and has recently been demonstrated effective at investigating permafrost in the subsurface [6]. In this area, EIT is also known by the names Electrical Resistivity Surveys and DC Resistivity Surveys. Typically, direct current (DC) is used for geophysical applications, as it is easier to generate and alternating current (AC) offers few advantages for earth materials [3]. An example of the earth cross-section from the permafrost investigation can be seen in Figure 1-1. As time progresses, the frostline recedes, which can be seen by the increase in conductivity near the surface. Here the lighter colors represent higher conductivities. To aid the viewer, a dotted line representing the frostline is drawn and can be seen to recede into the earth as the atmosphere warms seasonally. Depth and lateral extent is given in meters. 


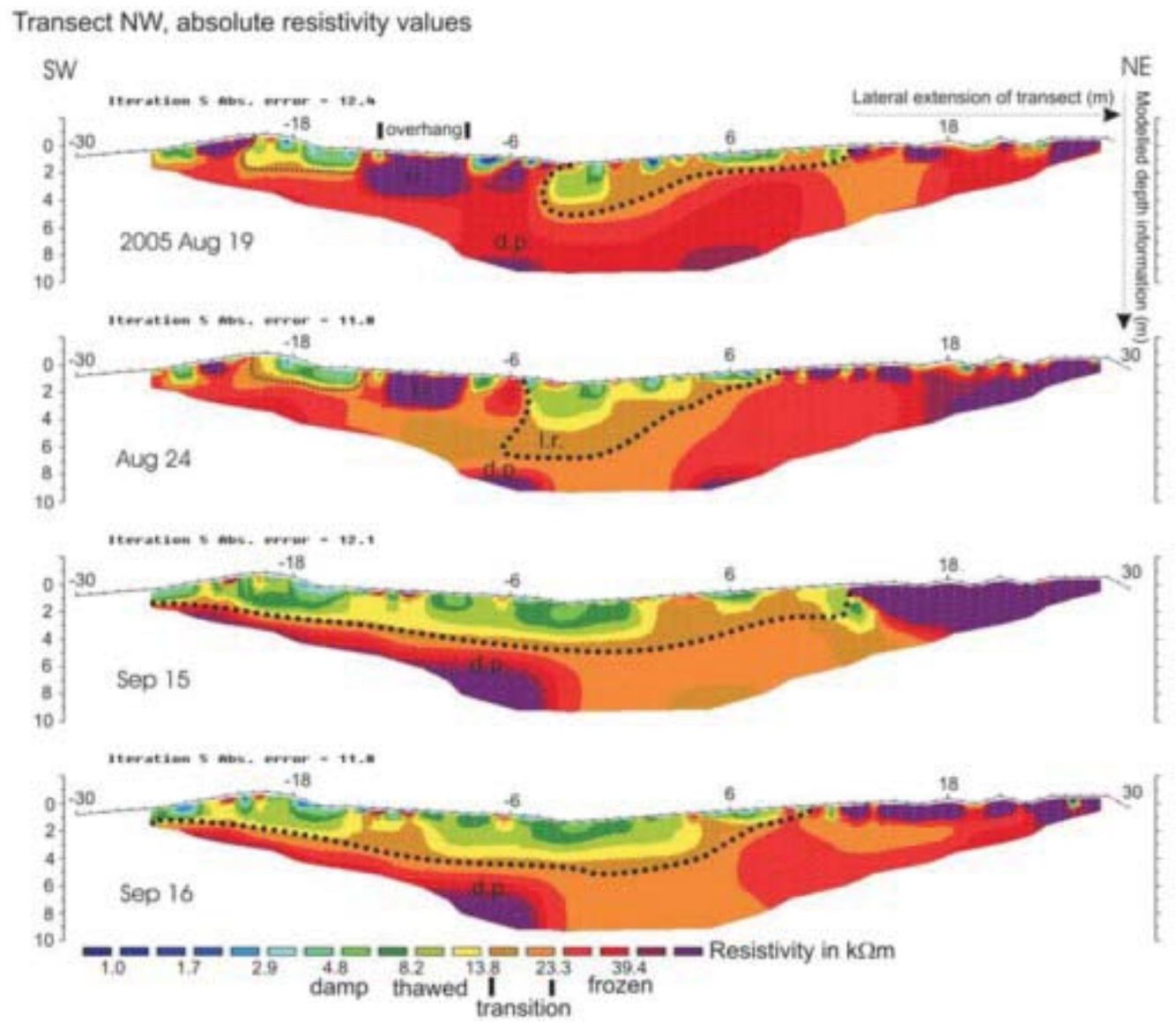

Figure 1-1 Resistivity cross section showing receding frost line reproduced from "Electrical resistivity tomography monitoring of permafrost in solid rock walls." Journal of Geophysical Research: Earth Surface (2003-2012) 112.F2 Copyright 2007 John Wiley \& Sons, Inc. This material is reproduced with permission of John Wiley \& Sons, Inc. See Appendix A for permission documentation.

In industrial applications, EIT has been used for flow monitoring [7], tank settling [8], and a system that is very physically robust has been developed for use in a nuclear fuel reprocessing plant[9]. Both $D C$ and alternating current $(A C)$ systems are used in this field, with $\mathrm{AC}$ sometimes being used at multiple frequencies to allow for simultaneous multiple measurements. An example of a tank settling experiment can be seen in Figure 1-2. Here, the conductive particulate settles, which can be seen by the decrease in 
conductivity as time progresses. High conductivity is shown as red and low conductivity is shown as blue.
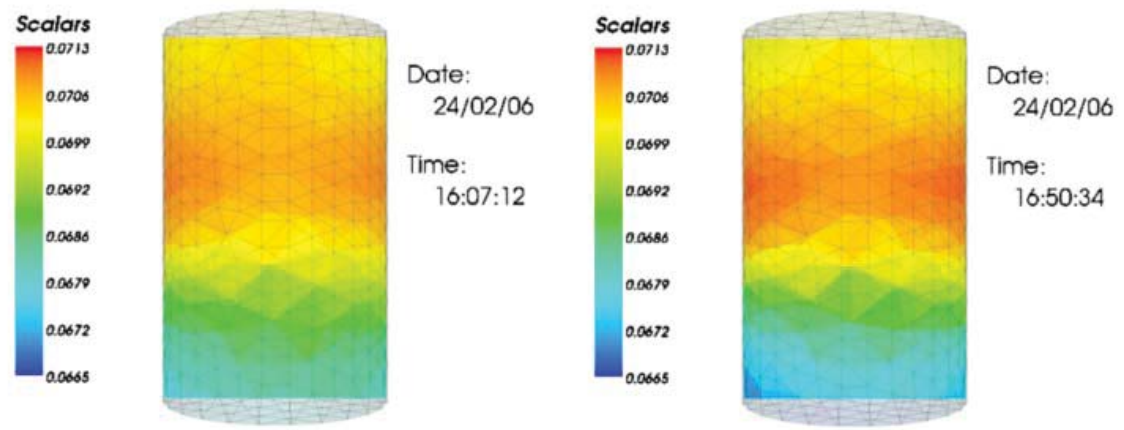

(a) $-8.5 \mathrm{mins}$

(b) -52 mins
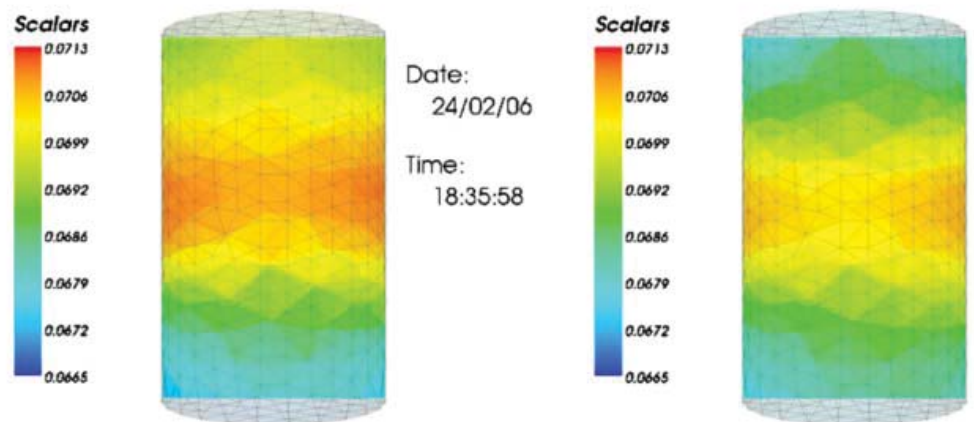

Date:

$24 / 02 / 06$

Time:

23:15:59

(c) -2 hrs 37.5 mins

(d) -7 hrs 17.5 mins

Figure 1-2 Time-lapse imaging of tank settling experiment taken from Murphy, S. C., et al. "3D electrical tomographic imaging using vertical arrays of electrodes." Measurement Science and Technology 17.11 (2006): 3053. ( IOP Publishing. Reproduced with permission. All rights reserved doi:10.1088/0957-0233/17/11/026 Permission documentation in Appendix A

In medicine, an EIT system is commercially available for the monitoring the lungs of ventilated patients [7] and research is ongoing to use the technology for a variety of uses including tumor detection and measurement of tissue blood perfusion[10]. Typically in this field, AC stimulus is used to take measurements, so that the patient will not experience discomfort from their tissues being heated [10]. Cheney, Isaacson, and 
Newell demonstrated the use of the technology for investigating a 2D cross section of an individual's chest. The apparatus and cross section from this study can be seen in Figure 1-3 and Figure 1-4. [10]. The lungs, filled with air, are very resistive and are depicted as red in Figure 1-4. The heart, filled with conductive blood, has a low resistivity and is depicted as blue.

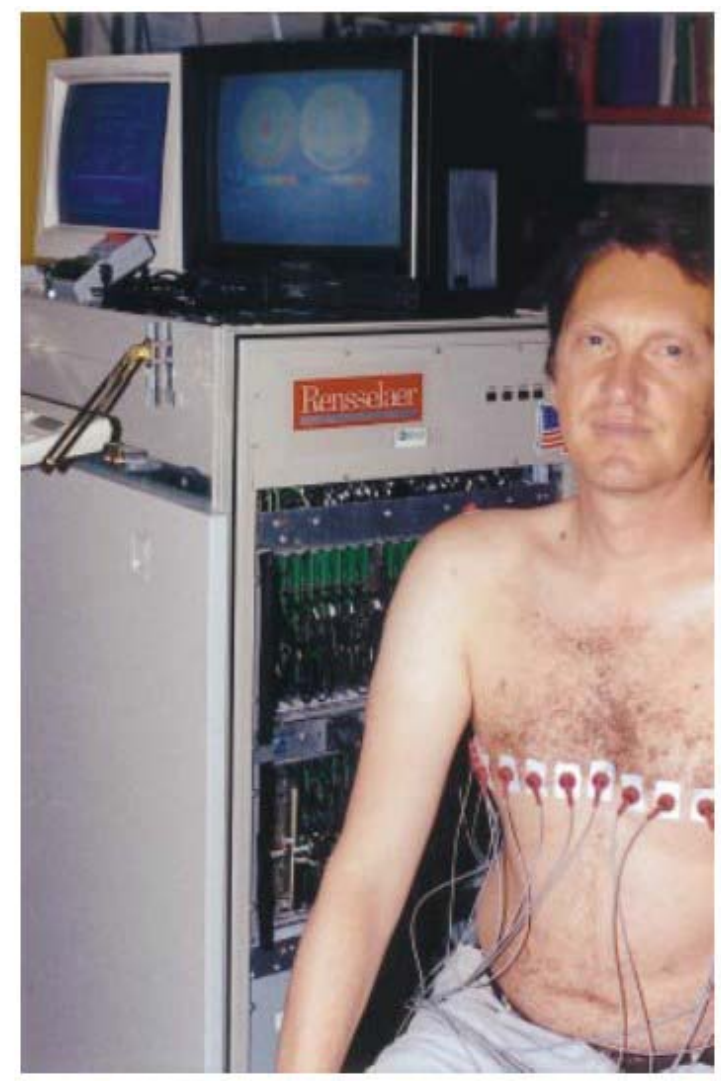

Figure 1-3 Electrical Impedance Tomography Device with electrodes attached to a patient's chest. Taken from Cheney, Margaret, David Isaacson, and Jonathan C. Newell. "Electrical impedance tomography." SIAM review 41.1 (1999): 85-101 Copyright @1999 Society for Industrial and Applied Mathematics. Reprinted with permission. All rights reserved. See Appendix A for documented permission 


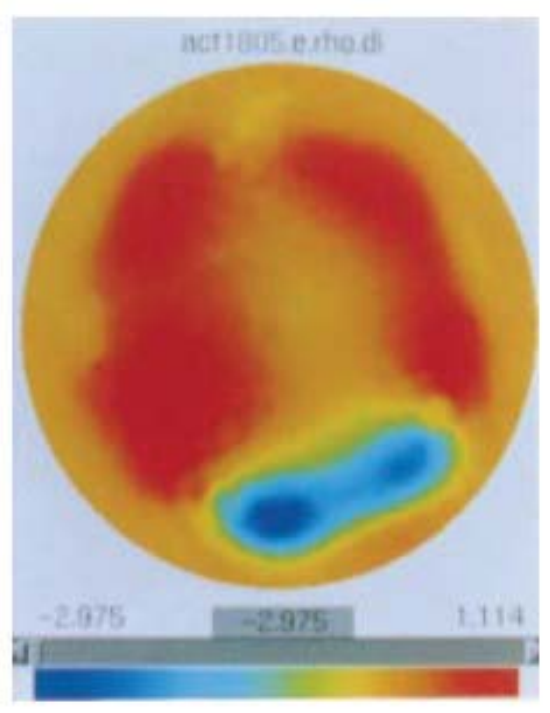

Figure 1-4 Reconstructed resistivity cross-section from patient in Figure 1-3. Taken from Cheney, Margaret, David Isaacson, and Jonathan C. Newell. "Electrical impedance tomography." SIAM review 41.1 (1999): 85-101 Copyright @1999 Society for Industrial and Applied Mathematics. Reprinted with permission. All rights reserved. See Appendix A for documented permission

\subsubsection{History of EIT}

EIT roots can be traced back to 1912, when the Schlumberger brothers showed that resistivity measurements could be used to explore for ore bodies beneath the surface of the earth[5]. They found that by varying the spacing of the electrodes, the measured, or apparent, resistivity would be indicative of different depths within the earth. By taking multiple measurements, they would map out the resistivity of the subsurface. The technique found even more use in 1930, when the Schlumberger brothers used it inside of an oil well to indicate the well depths from which oil was produced [11]. Over time, this specific test grew into the field of resistivity well logging, which is still performed on a 
large number of gas and oil wells after drilling, as it can be used to estimate porosity as well as water and oil saturation levels [12].

These early forays into electrical resistivity mapping were not tomography in the sense that they were reconstructing the subsurface from measurements. Instead, they focused on estimating the resistivity at a finite number of specific points in the subsurface. Researchers of the time found that as they moved the electrodes applying the current further and further apart, the equipotential lines became more affected by deeper heterogeneities. They devised a technique that is still in use today where each measurement is given a weighting factor based on the electrode positions. The technique involves taking each measurement and estimate a "pseudo-resistivity" from it, corresponding roughly to the resistivity at a depth and location determined by where the measurement took place. These pseudo-resistivities are then stacked together in pseudo-sections, which is a technique still used to check data quality during testing.[3]

\subsection{Ballistic Gelatin}

Ballistic gelatin was developed for the study of terminal ballistics (sometimes called wound ballistics), the term given to the behavior of a projectile entering, transferring energy to, and destroying living tissue or other targets. Ballistic gelatin is a pork gelatin engineered for consistent mechanical properties that simulate living tissue and excellent optical clarity [13]. It is a well-studied material with papers detailing its properties [14], the consistency of those properties [15], the factors that affect the consistency of those properties [16], and ways to improve the visualization of the cavities formed among other topics [17]. Of particular interest to the problem at hand are the consistency of the properties and factors affecting those. In [16], Jussila proposes a method for preparing 
gelatin that provides repeatable mechanical properties. Ballistic gelatin was selected for this study due to its optical clarity, low fracture strength, and conductivity.

\subsection{Fracture Mechanisms Studied}

\subsubsection{Terminal Ballistic Behavior}

Bullets are often designed to behave a certain way upon entering a target depending on the design intent of the bullet. Bullets have been designed that will expand, stay fully formed, fragment, intentionally tumble upon entry, or deliver a hardened core. For this study, we will focus on full lead .22 caliber bullets. The terminal behavior of these bullets is well understood and predictable.[18] The full lead .22 bullet will tumble at least once as it travels through the ballistics gel. Tumbling with a full metal bullet often occurs after penetration because the stabilizing effect of the bullet's spin becomes insufficient to maintain a point-forward travel after entering the denser medium of the gelatin.[19]

As the bullet enters the gelatin, it creates an entrance roughly the size of the bullet. As the bullet travels into the gelatin, kinetic energy is transferred to the gelatin forming a temporary cavity many times larger than the bullet diameter. After the temporary cavity reaches its maximum size, it will collapse towards its unstretched state and oscillate between states briefly. After the oscillation ends, a permanent cavity with radiating fractures will be left to show the path of the bullet. The permanent cavity will be of a slightly larger diameter than the projectile. If tumbling occurs, both of the cavities will be of a larger diameter than in the non-tumbling case and may not be radially symmetric. Additionally, the bullet's path through the gelatin will typically be linear, but may not be 
linear if tumbling occurs.[13] The typical behavior of a full metal .22 caliber bullet in ballistic gel is show in Figure 1-5.[20]

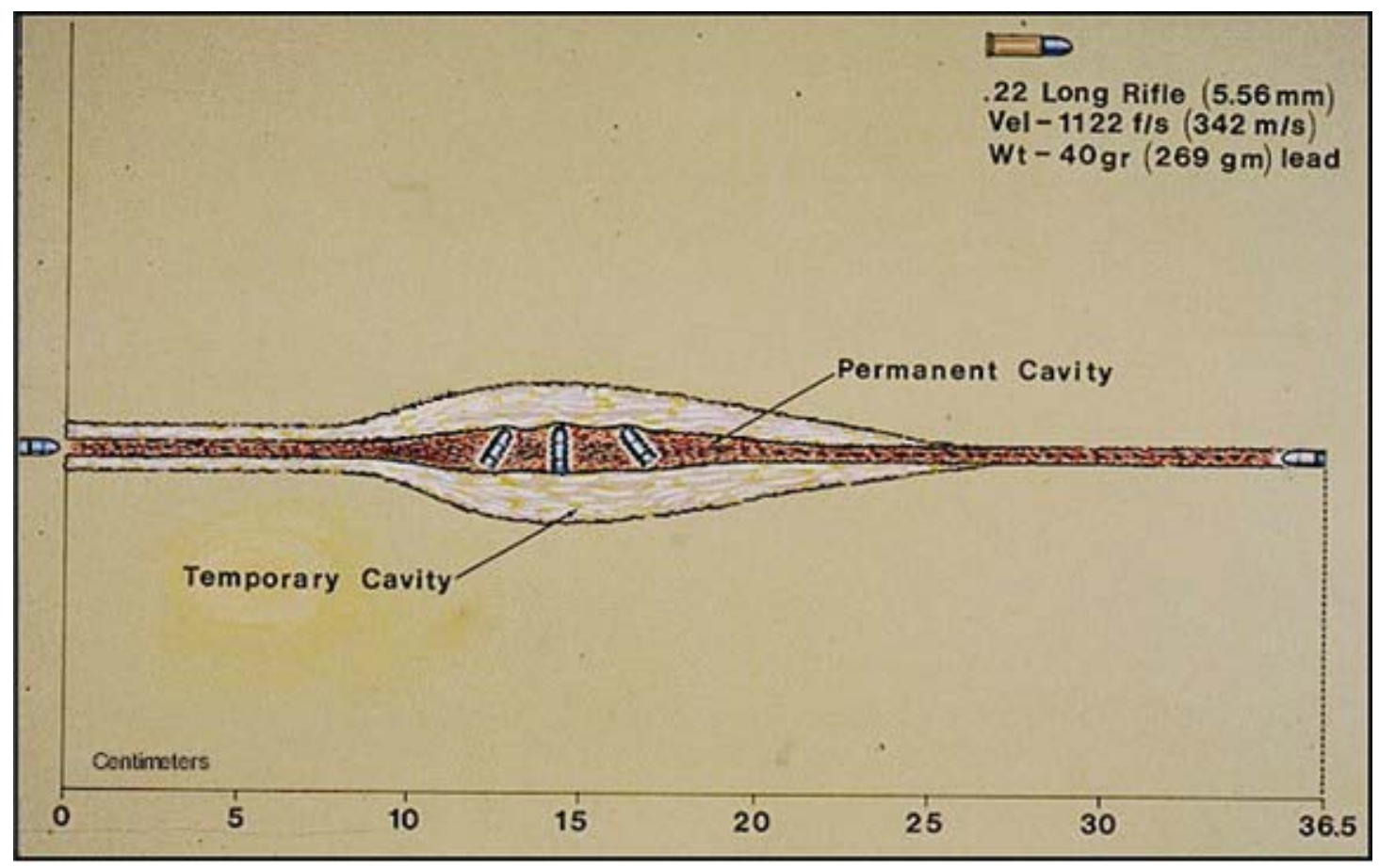

Figure 1-5 Terminal ballistic behavior of .22 caliber full metal bullet. Figure taken from Fackler, MARTIN L., and JOHN A. Malinowski. "The wound profile: A visual method for quantifying gunshot wound components." The Journal of Trauma 25.6 (1985): 522. ๑ 1985 Wolters Kluwer Health.

Reprinted with permission. See Appendix A for permission documentation.

Terminal ballistics is often studied by taking high-speed footage of the bullet-gelatin interaction. This allows measurement of temporary cavity formation and bullet behavior such as expansion, fragmentation, and tumbling. Permanent cavity diameter and bullet track (depth) measurements can be easily taken after the test. To improve visualization and allow for better estimation of the temporary cavity after a test, a method involving putting a bag of acrylic paint in the front of the gelatin block has been developed.[17, 21] 
This leaves a path of paint through the gelatin, helping discriminate the temporary cavity after it has closed.

\subsubsection{Hydraulic Fracturing}

Hydraulic fracturing is a commonly used method of accessing natural gas reserves that are not available through conventional methods. In hydraulic fracturing, fluid is pumped into a borehole and exerts pressure on the rock. The pressure causes the rock to fracture due to the added stress, which opens up pockets of natural gas that were trapped in impermeable rock. Granular sand crystals or small beads made of bauxite or ceramic materials, called proppant, are pumped down with the fluid to hold the fractures open after they have been opened. This allows the gas to flow more easily into the well. [22] Performing hydraulic fracturing experiments in gelatin is not a new concept and was first documented in [23].

\subsection{Objectives}

The objectives of this project are as follows:

- Develop a dynamic EIT system that is easily transportable and uses relatively inexpensive off-the-shelf hardware.

- Use the developed EIT system to evaluate static and dynamic fracture phenomenon as a function of time.

- Validate the results of the developed EIT system.

\subsection{Contributions}

This project contributes to three different fields: EIT, Geophysics, and Terminal Ballistics.

\subsubsection{Electrical Impedance Tomography}


The work performed here is directly contributing to the field of EIT by performing EIT on a new type of system. It is also contributing to EIT by developing and testing a new EIT system based on inexpensive off-the-shelf data acquisition (DAQ) hardware.

\subsubsection{Geophysics}

There is currently a large amount of interest in determining extent of hydraulic fractures. $[24,25]$ The method demonstrated here could be considered a proof-of-concept for future work on the use of physical scale models to test electrical resistivity based techniques to determine extent of hydraulic fractures.

\subsubsection{Terminal Ballistics}

This work demonstrates a method of high speed imaging in ballistics gelatin across planes that are difficult to visually image. This could be used to better understand the factors that play into temporary cavity formation. 


\section{Theory}

\subsection{Physics}

The two equations governing the physics of the electrical impedance tomography problem are Ohms law and Kirchhoff's current law[4, 26]. Ohm's law, stated in Equation 2.1 , tells us that the voltage across a resistive load is proportional to the current flowing through it. Voltage is represented as $\mathrm{V}$, current is represented as I and resistance is given as R. In a continuum, which is more useful as this is not a one dimensional problem, the equation is written as it is in Equation 2.2. Here, $\vec{J}$ is the current density, $\sigma$ is the conductivity, and $\vec{E}$ is the electric field. Conductivity and resistivity are inversely related to each other, as shown in Equation 2.3, and the terms are used interchangeably throughout this section. In Equation 2.3, resistivity is given as $\Omega$.

$$
\begin{gathered}
V=I R \\
\vec{J}=\sigma \vec{E} \\
\sigma=\frac{1}{\Omega}
\end{gathered}
$$

Kirchhoff's current law (KCL) states that current is conserved at junctions: in other words, the current flowing into a junction must be equal to the current flowing out of a junction. If only a finite region is considered, the problem can be explained as the 
divergence of the charge density being equal to zero. This can be written in equation form, as seen in Equation 2.4.

$$
\nabla \cdot \vec{J}=0
$$

\subsubsection{Conceptually understanding Electrical Impedance Tomography}

To understand how EIT works, it is useful to gain a conceptual understanding of how the governing equations are affected by changes to the system. This section will go through a series of simple FEM simulations to qualitatively explain the physics of EIT. Picture a two or three dimensional homogenous system, such as the two dimensional one pictured in Figure 2-1, which has a voltage applied between two points on the boundary. The scales are left dimensionless intentionally, as these diagrams are only meant for conceptual understanding. The voltage, or potential, will decrease between the two electrodes as it is shown in Figure 2-2. The lines of current flow can be drawn between the points that the current is applied between, which is shown in Figure 2-3. Lines of constant voltage can be drawn across the system and will lay orthogonal to the current flow lines as a consequence of Equation 2.2. Figure 2-4 has the lines of constant voltage

drawn into the previous picture and the right angled intersections can be seen between the constant voltage and current flow lines. 


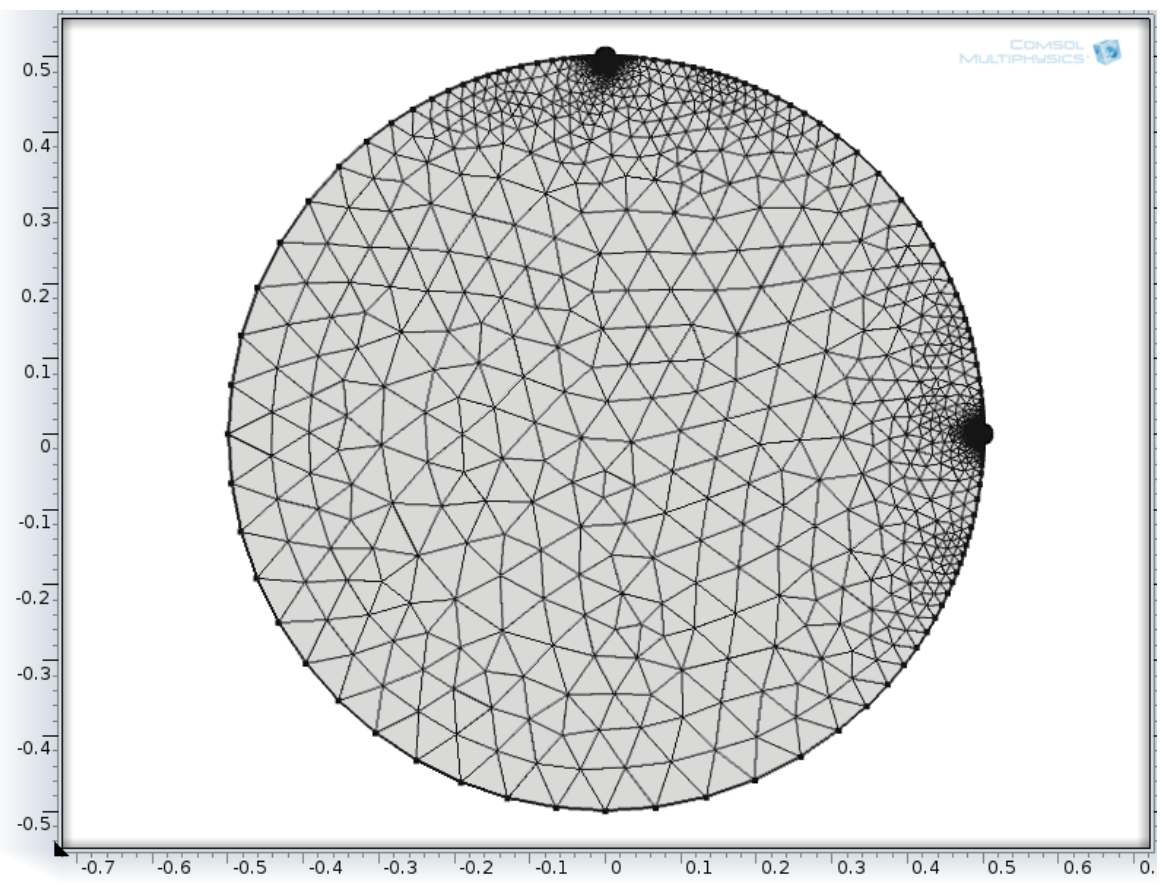

Figure 2-1 Finite element mesh used for homogeneous calculations

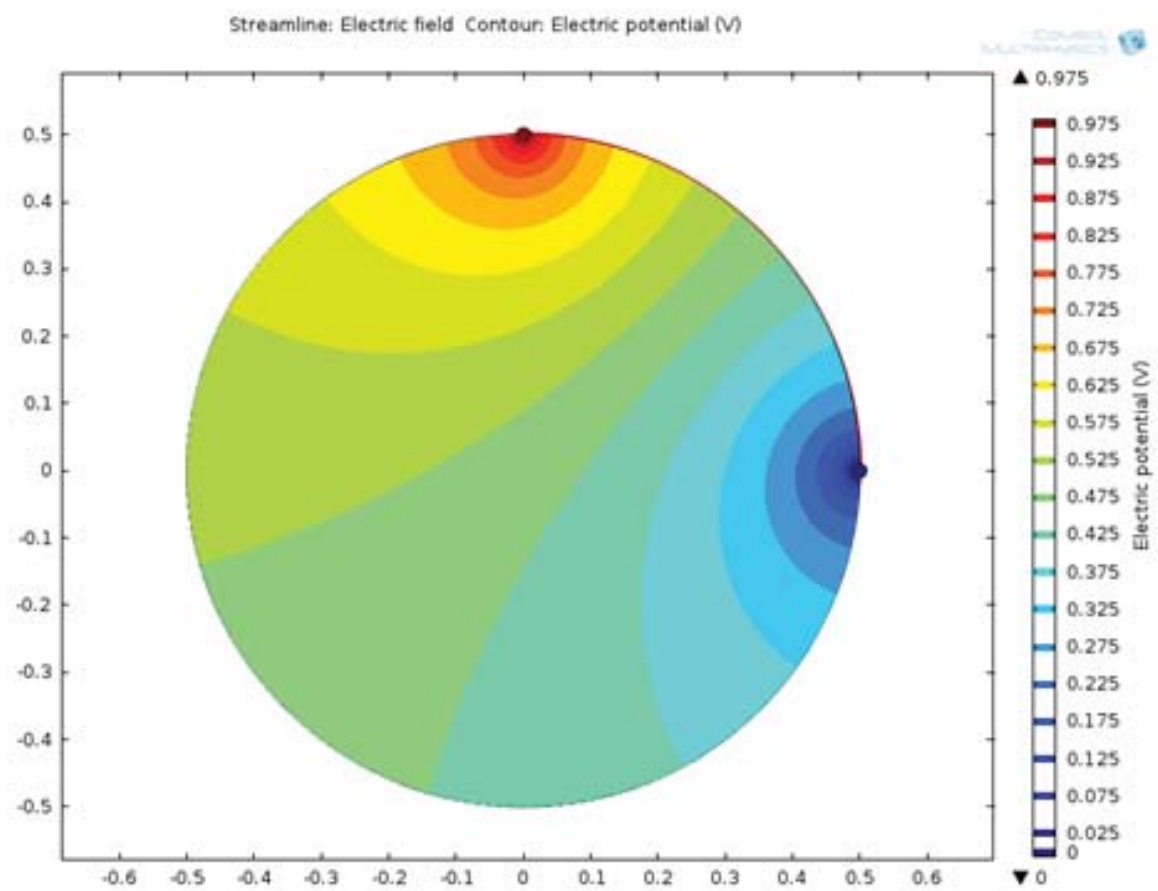

Figure 2-2 Voltage potential between electrodes for homogeneous model 


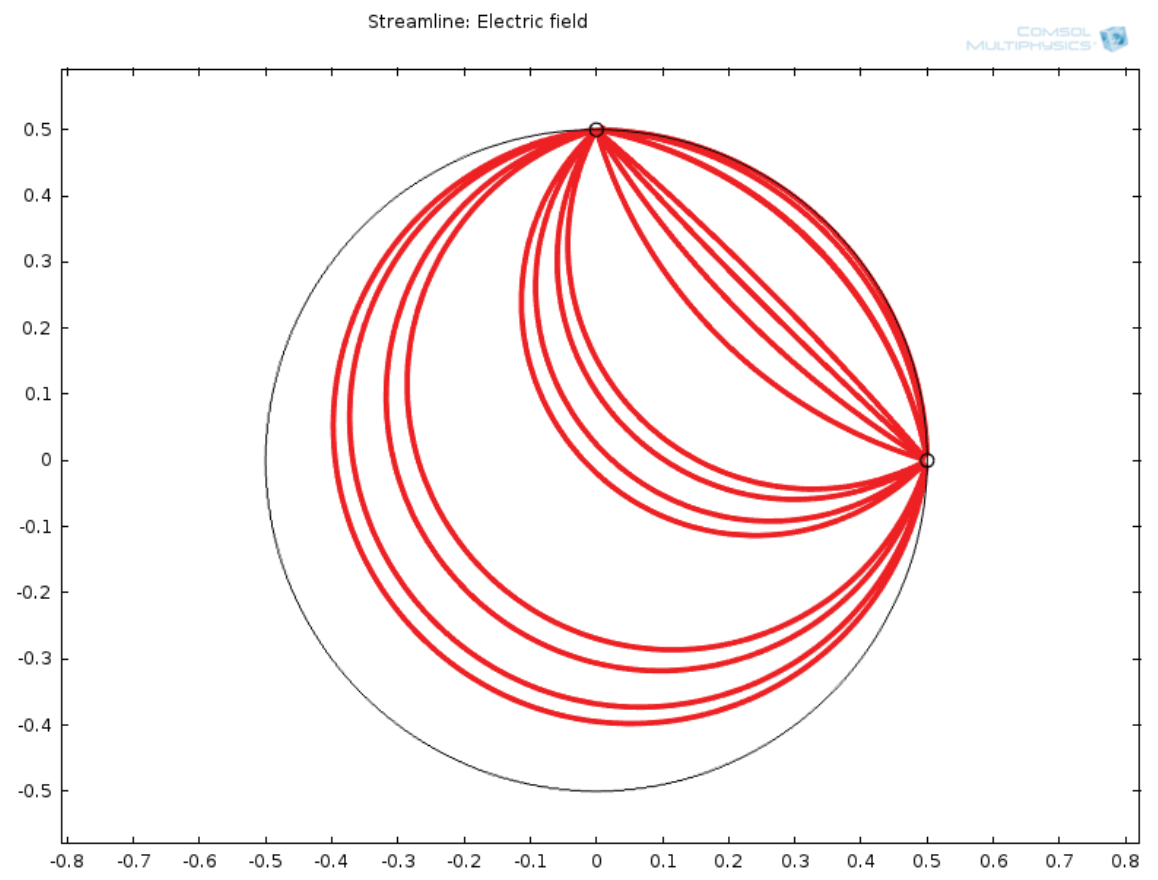

Figure 2-3 Current streamlines between electrodes for homogenous model

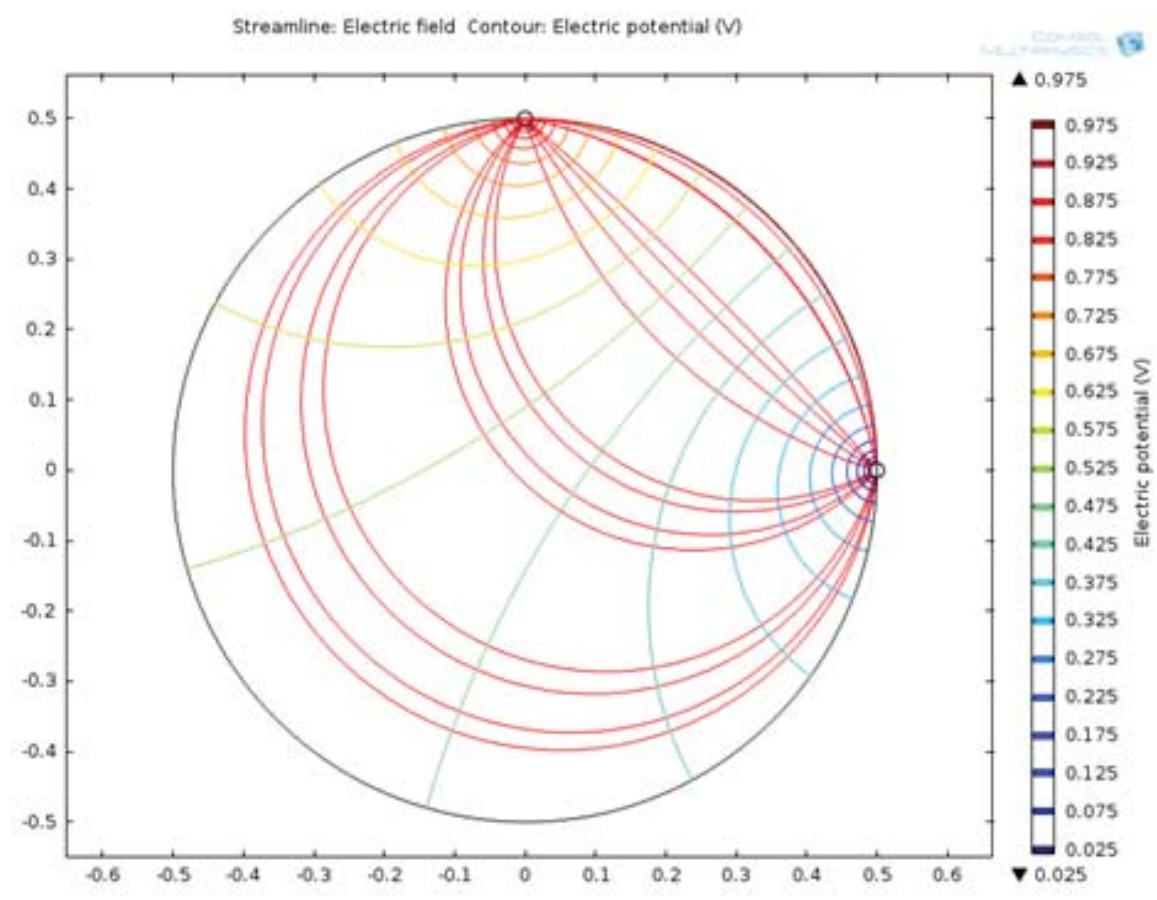

Figure 2-4 Current streamlines superimposed on voltage contours for homogeneous model 
If the system was changed from a homogenous one to one with heterogeneities, such as the model shown in Figure 2-5, the lines of current flow would change from the smooth curves seen in Figure 2-3 to one where the path of least resistance may involve a different path. Figure 2-6 contains a more conductive heterogeneity and Figure 2-7 contains a less conductive one to demonstrate how the current streamlines will behave differently. Figure 2-8 and Figure 2-9 show the effect of a more conductive heterogeneity and a less conductive heterogeneity on the voltage potentials.

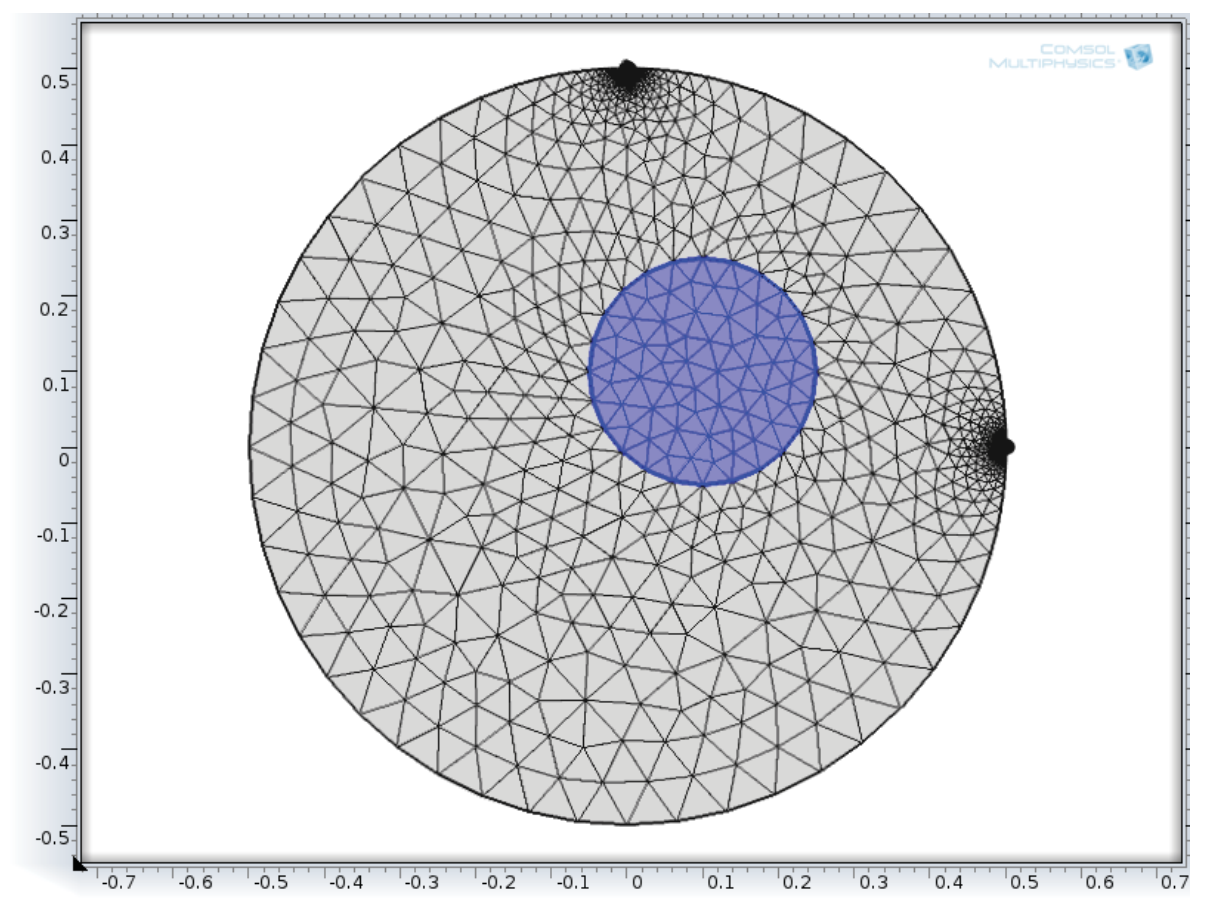

Figure 2-5 Generic finite element mesh with heterogeneity used for calculations 


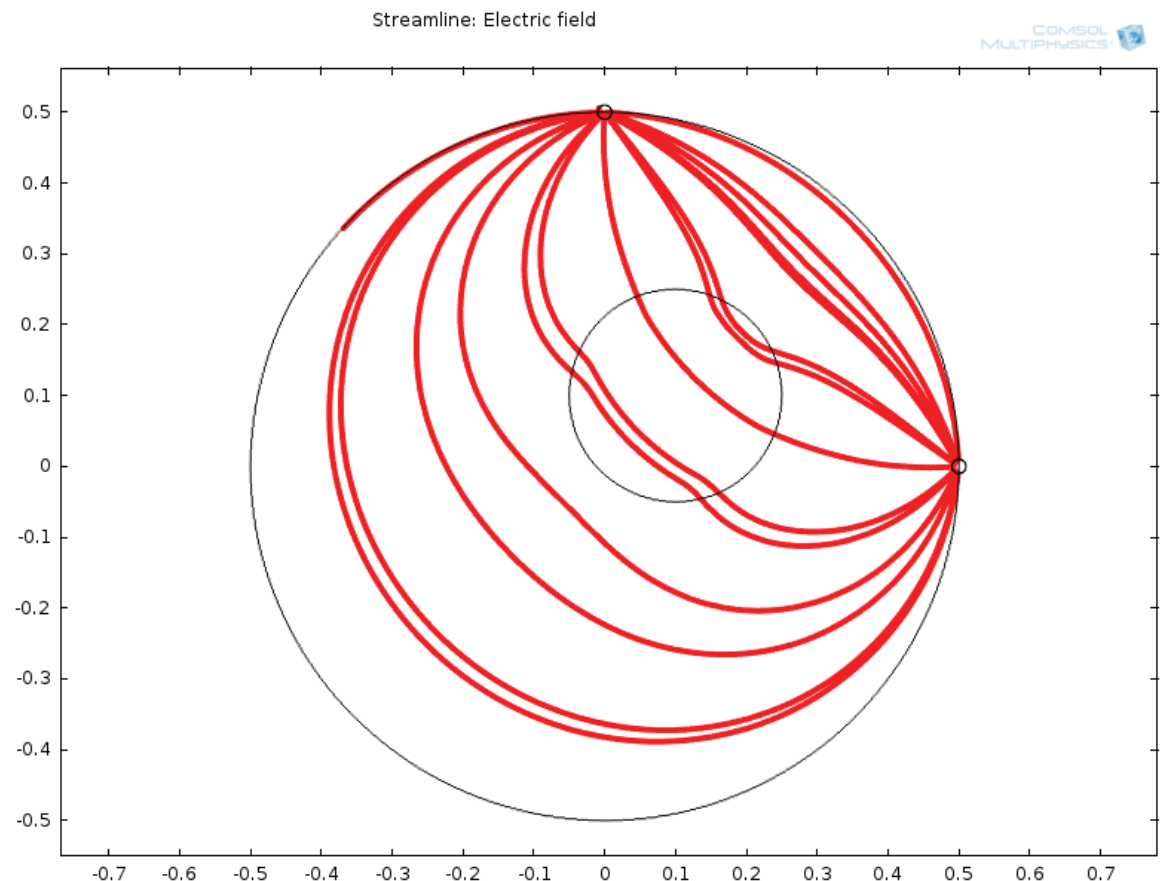

Figure 2-6 Current streamlines showing effect of more conductive heterogeneous inclusion

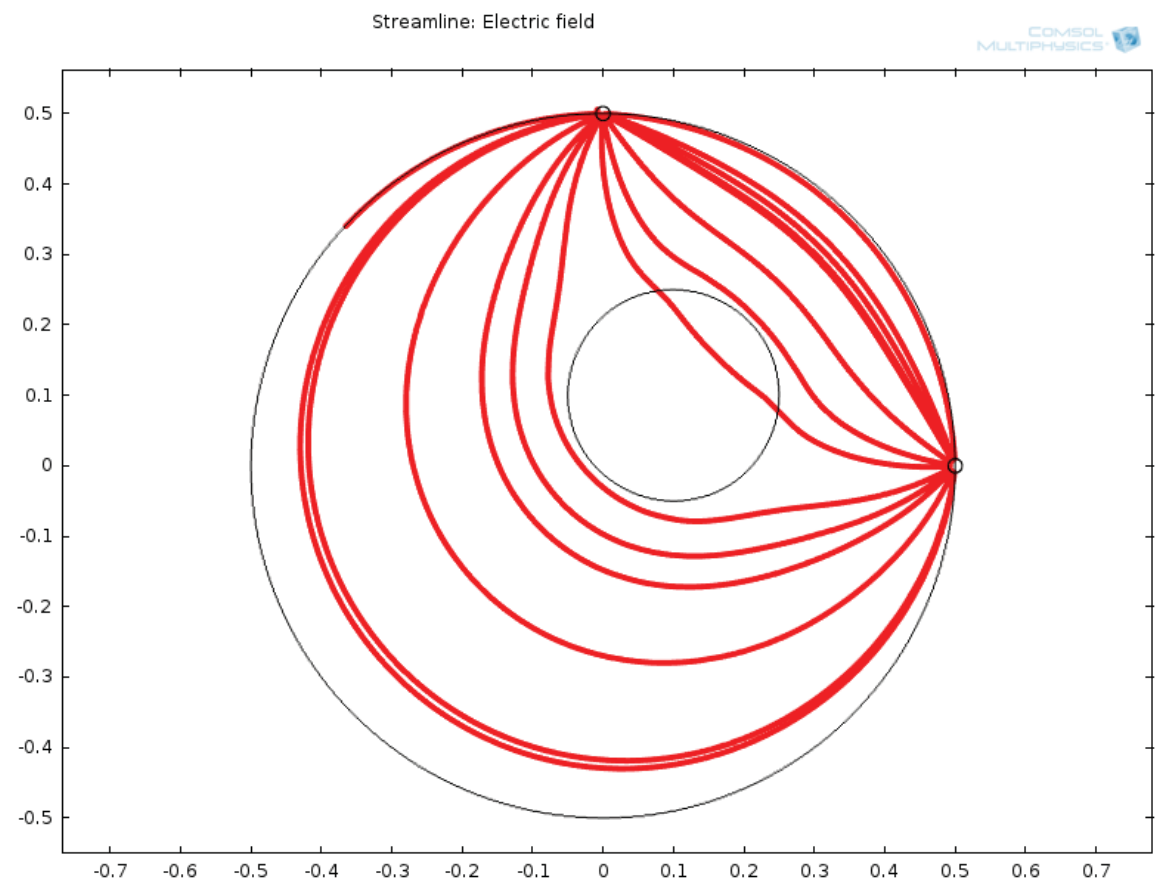

Figure 2-7 Current streamlines showing effect of less conductive heterogeneous inclusion 


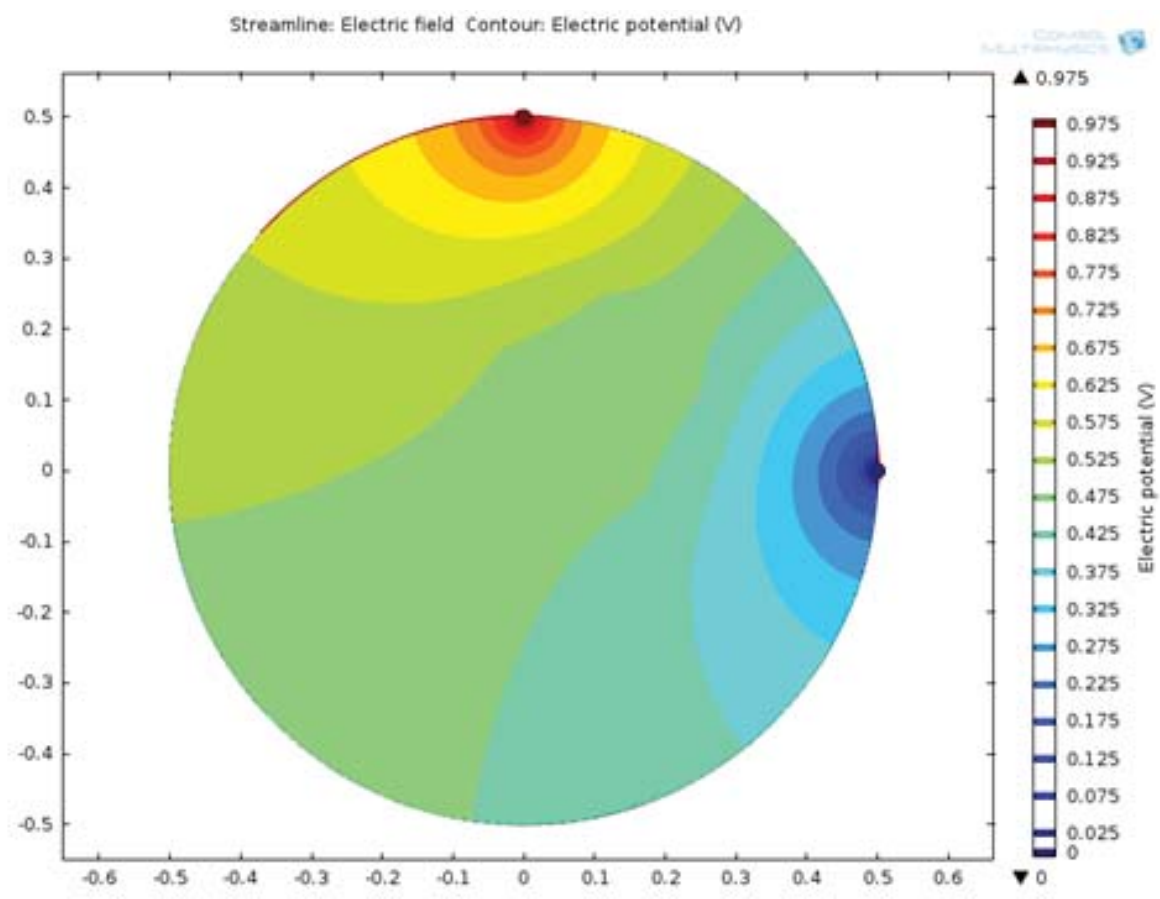

Figure 2-8 Voltage contours showing effect of more conductive heterogeneous inclusion

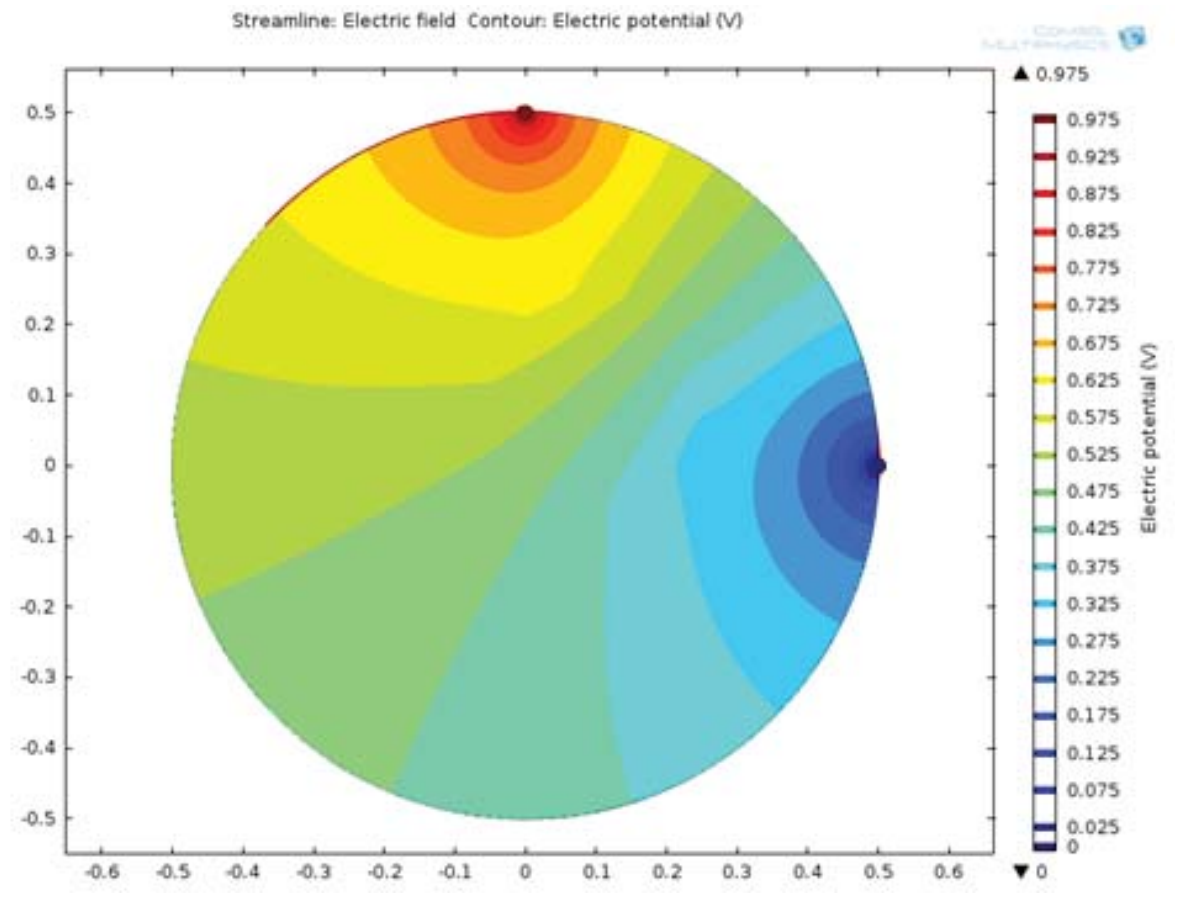

Figure 2-9 Voltage contours showing effect of less conductive heterogeneous inclusion 
As can be seen in the preceding figures, the lines of constant voltage are changed by the introduction of the heterogeneity and are disturbed up to the boundary of the system. This is the key to how electrical impedance tomography works: changes to the resistivity of the interior of a system will influence voltage measurements taken on a system boundary when a current is applied to the boundary.

\subsubsection{Comparison of EIT to Other Tomographic Imaging Methods}

Because of the multi-path nature of the electrical current, EIT is considered a soft-field tomography[7]. Tomography is defined as measuring the propagation of energy or particle motion in order to reconstruct information about the interior of the system. Usually this information is a reconstruction of a physical property of the system, such as density or resistivity. A soft-field tomography technique is defined as being a tomography technique that relies on a multi-path propagation. Besides EIT, a common example of a soft-field tomography is crosswell seismic tomography. In crosswell seismic tomography, a seismic source generates vibrations at various positions in a borehole and a series of geophones or hydrophones measure the response in an adjacent borehole. A hard-field tomography technique is one where each measurement is influenced by a known, finite region. A good example of a hard-field tomography technique is Computed Tomography, where an X-ray is shot into a section of a system (generally a human) from a range of different angles. By measuring the signal attenuation at the other side and knowing the beam width, it is possible to reconstruct a slice of the system's attenuation properties, which are related directly to density. 


\subsection{Mathematics}

\subsubsection{Overview of the reconstruction problem}

Mathematically, EIT reconstruction generally consists of both an inverse problem and a forward problem[7, 27]. The inverse problem relates the measurements to the model. The forward problem is used to develop the sensitivity matrix, or Jacobian, that is used in solving the inverse problem. A generalized graphical representation of this can be seen in Figure 2-10. Specific reconstruction techniques vary in how the inverse and forward problems are solved. Additionally, some reconstruction methods are iterative, where the Jacobian is recalculated from the updated model. The equations used for this project will be described below in Section 2.23 and Section 2.24 .

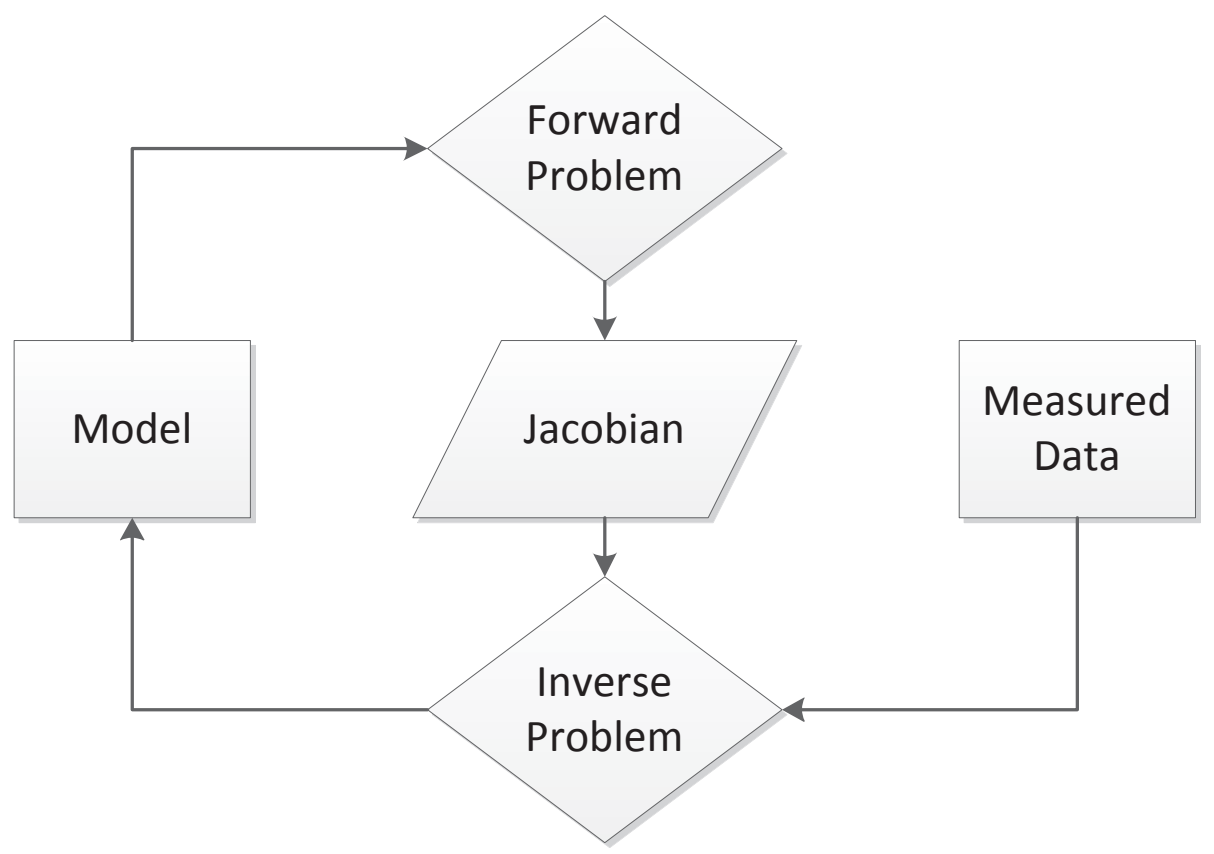

Figure 2-10 Graphical representation of general EIT reconstruction method

Four major classes of imaging in EIT were identified in [4] , with the type encountered depending on the project requirements. Time difference imaging, the most common 
type, involves taking a set of measurements from an undisturbed system and comparing them to measurements of a system that has undergone some sort of change. The results of this sort of imaging will be the change in resistivities of the system. Results from solving the forward model can be used in place of the undisturbed system measurements. Time difference imaging is computationally much easier and becomes linear in some inversion schemes.

The other three imaging modalities are: static imaging, multi-frequency imaging, and dynamic imaging. Static imaging is used to find the absolute resistivity distribution from a set of measurements. Multi-frequency imaging involves performing either time difference or static imaging with multiple excitation frequencies. This allows the complex impedance distribution of a system to be estimated, which may be advantageous for certain medical applications where tissue properties are related to the capacitance of the tissue. Dynamic imaging generally involves the implementation of a Kalman filter to make estimates from partial measurement sets, allowing resistivity distribution estimates to be made at a faster rate than in other methods.

\subsubsection{Electrical Impedance Tomography and Diffuse Optical Tomography Reconstruction Software}

All calculations in the reconstruction problem were performed using EIDORS, which is short for Electrical Impedance Tomography and Diffuse Optical Tomography Reconstruction Software. EIDORS is a codebase for MATLAB (or Octave) that contains code for both the forward and inverse problem of EIT. It is licensed under the GNU General Public License and is free to use [28]. Other code for inversion is freely available, but is focused on geophysics applications[29, 30]. Additionally, EIDORS has a very active community that is continually developing and sharing new code. It supports 
Netgen and Distmesh for three- and two-dimensional meshing, respectively; both of which are freely available online.

\subsubsection{The Forward Problem}

The forward problem is concerned with predicting the values of the observable parameters given an input. This is accomplished using a Finite Element Method (FEM), where each element is assigned a conductivity. The elements are based on the predicted change in voltage potential, with more elements placed where voltage potential is expected to change faster. Due to the geometrical spreading of the current lines combined with Equation 2.2, this means that the areas around the electrodes supplying current must have a greater element density. To simplify the problem and decrease computational time, the same mesh is used for all sets of measurements.

Finite Difference Methods (FDM) and Boundary Element Methods (BEM) can also be used, but are not preferred. FDM suffers computationally when geometry and boundary conditions become complicated. BEM becomes very inefficient when the reconstructed resistivity structure is no longer made of smooth surfaces.[1]

\subsubsection{The Jacobian}

The Jacobian, sometimes called the sensitivity matrix, relates the change in conductivity of an element to the change of a measurement. With complicated geometries, it is often calculated by perturbing each element of the finite element mesh and solving the forward problem for the change in the boundary voltage measurement. For the two-dimensional finite element models used in this project, the Jacobian was calculated analytically for each element using Equation 2.5. 


$$
\frac{\partial V_{d m}}{\partial \sigma_{k}}=\int_{\text {element }_{k}} \nabla u\left(I^{d}\right) \cdot \nabla u\left(I^{m}\right) d V
$$

\subsubsection{The Inverse Problem}

The inverse problem in EIT is particularly difficult, being ill-conditioned, rank-deficient, and ill-posed[7]. The rank deficiency can be understood as having an insufficient number of measurements. The ill-conditioning refers to the ratio between largest and smallest value. The ill-posedness refers to Hadamard's criteria for well-posed problems. The criteria states that a solution must exist, that the solution must be unique and that the solution should change continuously with changing conditions.[24] Physically, an illposed problem is highly sensitive to small changes and will require regularization to obtain a realistic solution. $[31,32]$

In generalized terms, the inverse problem is written as it is in Equation 2.6, where G is the system, $m$ is the model, and $d$ is the measured data. In the case of the EIT inverse problem, $m$ is the resistivity of each finite element, $d$ is the measured data, and $G$ is a function of the conductivity of the system. In the case of difference imaging, $G$ is the Jacobian of the system and its calculation is discussed in Section 2.2.4.

$$
[\mathrm{G}]\{\mathrm{m}\}=\{\mathrm{d}\}
$$

Because $G$ will not be a square matrix, it is not possible to directly invert it. The easiest approach to this is the Moore-Penrose inverse or pseudo-inverse. Mathematically it is 
expressed as can be seen in Equation 2.7 and is equivalent to the least-squares solution. This is a straightforward solution to the inverse problem and guarantees that a solution will be found. It suffers from being susceptible to ill conditioning and may provide erroneous results if a problem is very ill conditioned. It will not provide a reasonable solution if measurement errors do not follow a normal distribution. [31]

$$
\{\widehat{m}\}=\left([G]^{T}[G]\right)^{-1}[G]^{T}\{d\}
$$

The most basic improved technique for ill-conditioned systems is the truncated singular value decomposition (TSVD) approach. Condition of the system is often quantified by the condition number, which is defined as the ratio of the largest singular value to the smallest. Singular value decomposition (SVD) is a tool used to break apart matrices and analyze the rank, or linear dependency, of them. In this technique, small singular values, which are sensitive to noise, are truncated to reduce the condition number. After the smaller singular values are truncated, the system matrix is reconstructed in a pseudo inverse. Although this improves the conditioning, and hence the stability of the solution, it reduces resolution and is not an unbiased estimator.

Generally, a condition number under 100 is considered desirable. The unconditioned Jacobian matrices encountered in this project typically had condition numbers in the order of $10^{16}$ or $10^{17}$. A sample Jacobian mapped onto the finite element model for the linear section can be seen in Figure 2-11. The Jacobian indicates the sensitivity of each element in terms of measured voltage (at the measurement electrodes) per change in conductivity (or resistivity if formulated as such), given a set amount of stimulus current between another pair of electrodes. 

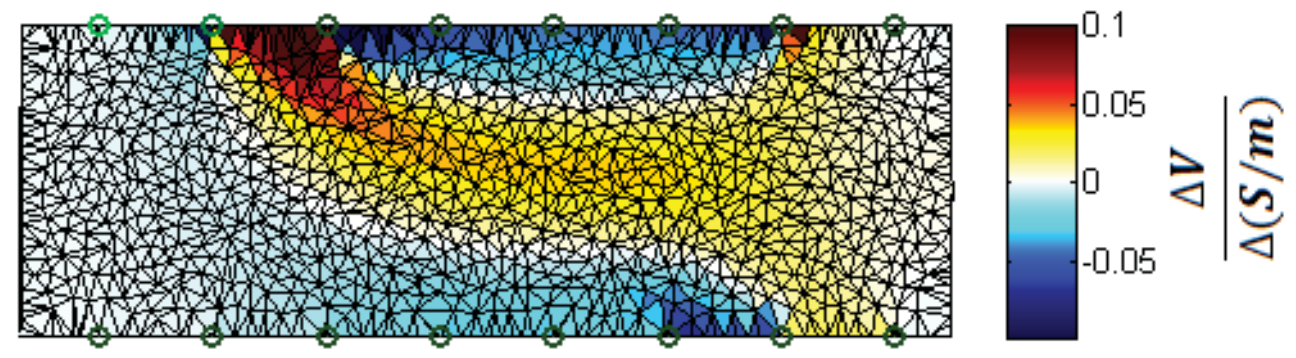

Figure 2-11 Sample Jacobian mapped onto FEM

Another way of handling the ill-posed and very ill-conditioned problem is to impose some constraints upon the solution to ensure stability. In the literature, this is referred to as regularization. Many regularization techniques exist, each of them optimized for a specific type of system. Generally, the regularization technique is chosen based on the expected result. For example, in the human body, it is expected that resistivity changes will be sudden between organs. For this reason, regularization techniques have been developed that favor sudden changes. More common are regularization techniques that smooth the reconstruction. These are less computationally intensive and do not always require iteration.

Tikhonov Regularization is the most common regularization method and has been applied to EIT by multiple researchers.[33, 34] It aims to improve upon the least squares 
solution by adding a penalty term that will cause the solution, $\hat{m}$ to be frequency limited. The matrix that provides this is called the Tikhonov matrix and is represented by $[\Gamma]$. Often in EIT, the Tikhonov matrix is an identity matrix scaled by a constant. The solution form of this equation is given in Equation 2.8. In EIT, the Tikhonov matrix is selected based on prior information known about the system's physical properties. [35]

$$
\{\widehat{m}\}=\left([G]^{T}[G]+[\Gamma]^{T}[\Gamma]\right)^{-1}[G]^{T}\{d\}
$$

The One-Step Gauss Newton Method is a non-iterative method that was selected for this project due to its demonstrated good performance in difference imaging. It performs similarly to the Tikhonov Regularization, but is formulated slightly differently as can be seen in Equation 2.9. The hyperparameter, $\lambda$, controls the smoothing introduced into the problem by adding directly to the diagonal elements of the pseudo-square Jacobian. As mentioned previously, performing iterations is the only way to determine estimates of absolute resistivity, however a one-step approach can be used to achieve reconstructions that will have geometrically similar resistivity distributions to the absolute resistivity. [24]

$$
\{\widehat{m}\}=\left([G]^{T}[G]+\lambda^{2}[I]\right)^{-1}[G]^{T}\{d\}
$$

\section{Results and Discussion}

\subsection{Test Apparatus}

Three separate test apparatuses were constructed for this project. The first consisted of two electrode arrays built into a polycarbonate pipe. The pipe was used as a water tank 
and as a mold/test fixture combination with ballistics gelatin. The electrodes were constructed from stainless steel cylinder stock and glued into the wall of the pipe with silicone caulk. One array was a 16 point ring located a third of the way from the top of the pipe and the other array was an 18 point linear array spread across one side of the entire 34.5 " tall pipe. This test apparatus was only used briefly, as it was found that the polycarbonate enclosure was not strong enough to contain the dynamic expansion of the ballistics gelatin during testing. A diagram of the initial apparatus can be seen in Figure 3-1.

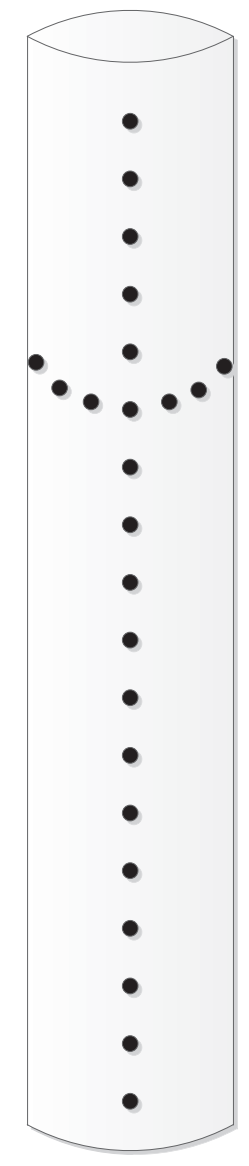

Figure 3-1 Diagram of original test apparatus showing electrode locations 
The other two test apparatuses use strips of non-conductive rubber to hold stainless steel carriage bolts onto the test samples. One apparatus was a 16 point ring array and the other was a pair of eight point linear arrays to be aligned across from each other. See Figure 3-2 and Figure 3-3 for diagrams of how the two arrays were wired.

The ring array used a stimulation and measurement pattern where electrode pairs were chosen as being one electrode less than 180 degrees apart based on the work of []. An example of this is in Figure 3-2, although only 8 electrodes are pictured for clarity. The actual model includes 16 electrodes. This array configuration was selected as it is a wellstudied array and has a proven ability to resolve resistivity inhomogeneities located throughout the plane the array intersects. 


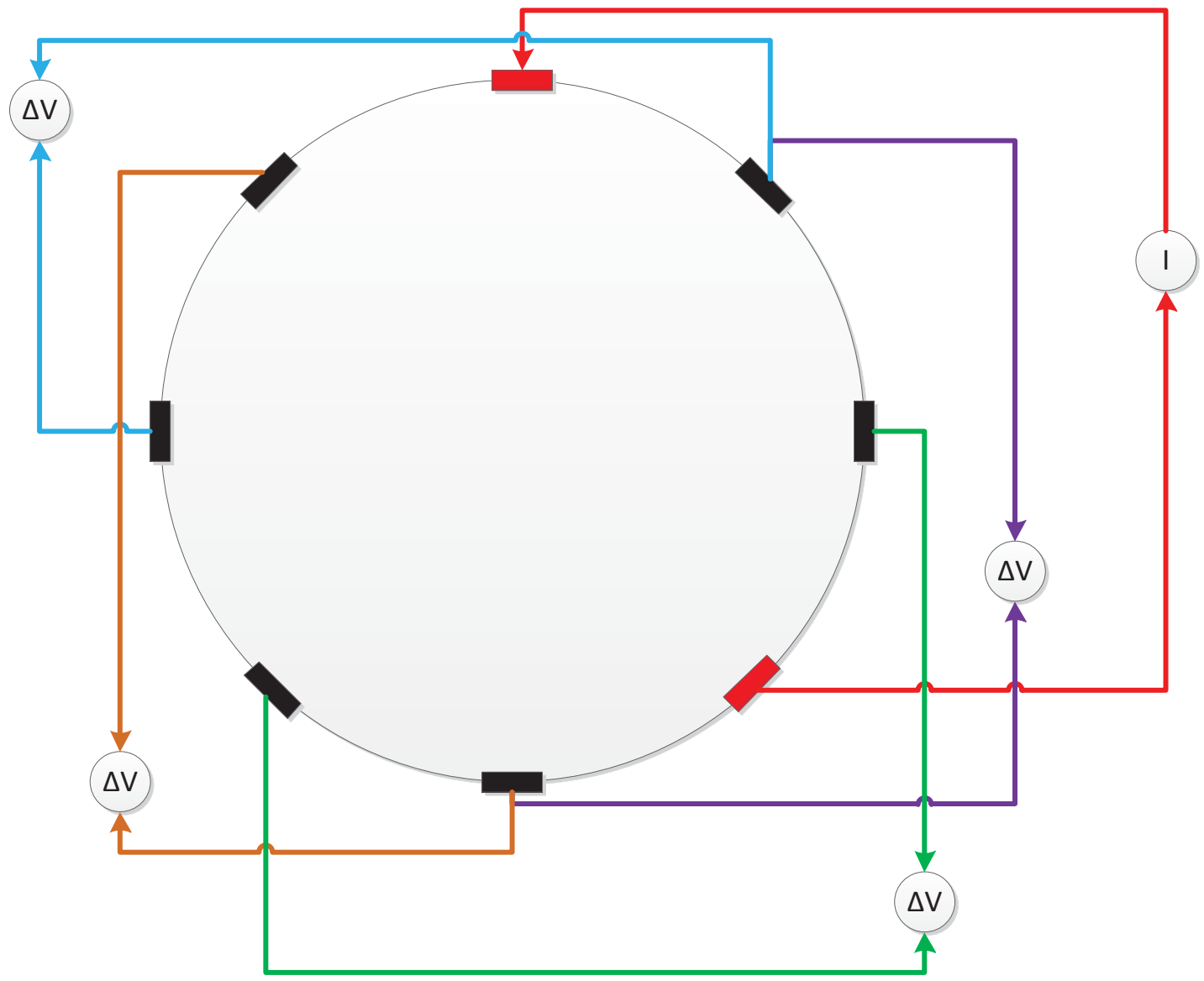

Figure 3-2 Example stimulation and measurement pattern for eight electrode ring array

The double linear array uses a stimulation and measurement pattern where opposing electrodes are driven and voltage is measured at adjacent electrodes on each side. See Figure 3-3 for an example of this. This was shown by [8] to be an effective system for resolving centrally located inhomogeneities. A triple linear array was shown to be superior in this paper, but due to hardware constraints this was not achievable for this study. 


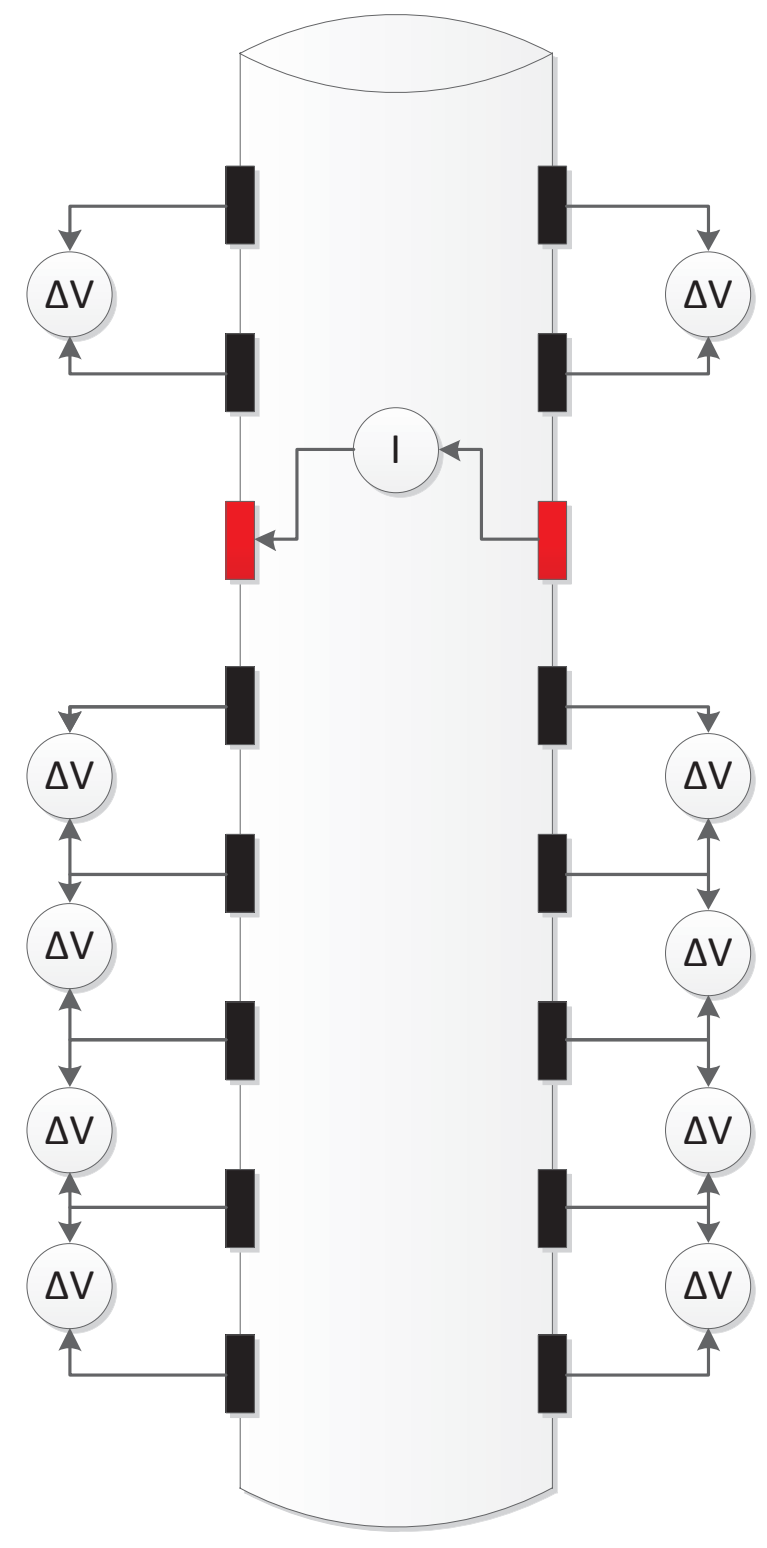

Figure 3-3 Stimulation and measurement pattern for double linear array

In both cases, measurements were not used from the drive electrodes. This would skew the results, as it would introduce a very sensitive region adjacent to the drive electrodes. Instead, data was recorded continuously from the electrode pairs as the drive electrodes cycled. This will be explained in more detail in the following section. 


\subsection{Data Acquisition and Processing}

The data acquisition hardware (DAQ) used for this study was a National Instruments (NI) 9172 chassis with NI-9234 analog input cards and NI-9263 analog output cards. The NI9234 input cards were the limiting factor for speed with this test, as they have a maximum sampling rate of 51,200 samples per second. The NI-9263 output cards were the limiting factor for capture time, as they could only hold 120,000 samples and run at the rate the input cards were configured to run at.

In most situations, EIT is performed using current sources for stimulation and voltage measurements. In the place of current sources, voltage sources were used for this experiment due to limitations on available hardware. Other researchers have experimentally demonstrated a method that allows the use of voltage sources and this method has been adopted for this research. The method demonstrated requires an iterative approach combined with some assumptions of small changes to the system.[36] The approach taken for this work assumed negligible changes in stimulus current (due to small changes in the system) and did not iterate.

\subsubsection{Hardware complications of the system developed for this study}

The system used for this study was limited by the hardware available, as there was no budget to develop a system from scratch or purchase a new system. One complication of this is that there were no computer-controllable current sources or sufficiently fast multiplexers available. A literature search found that the expense and availability of computer-controlled current sources was a problem identified by other researchers, who suggested a method by which voltage sources could be used as current sources.[36] To 
bypass the lack of a multiplexer, the number of electrodes was limited to the number of input channels on the available DAQ. To demonstrate the validity of the voltage source method, a current source was used to determine if the results would correlate.

\subsubsection{Data Processing Workflow}

Data was acquired continuously at the highest acquisition rate available on the selected DAQ with each channel measuring the voltage difference between a pair of electrodes. For the double linear system, this meant that there were 14 channels being recorded continuously. A sample of some raw data from a "practice" run can be seen in Figure 3-4. It can be seen that the stimulation pattern repeats itself over and over as time progresses. The data shown here was not taken at the same rate that the other reported test data was, but it provides a good example. The large spike in blue seen near the 15 second mark is the camera trigger, indicating that the high-speed camera had started recording. The red box indicates the data that is shown in Figure 3-5, which is a zoomed view of the raw data. The measured voltage difference is averaged across each pulse and converted to a single number that is tied to specific "measurement number", which indicates which electrodes were stimulated and which electrodes were measured. An example of two measurement vectors is given in Figure 3-6. It is also worth noting the subtle difference between the two measurement vectors, as this gives an indication of what ill-posedness physically means. A significant physical change, a large fracture, changes only a handful of the measured voltages and only changes them a small amount. 


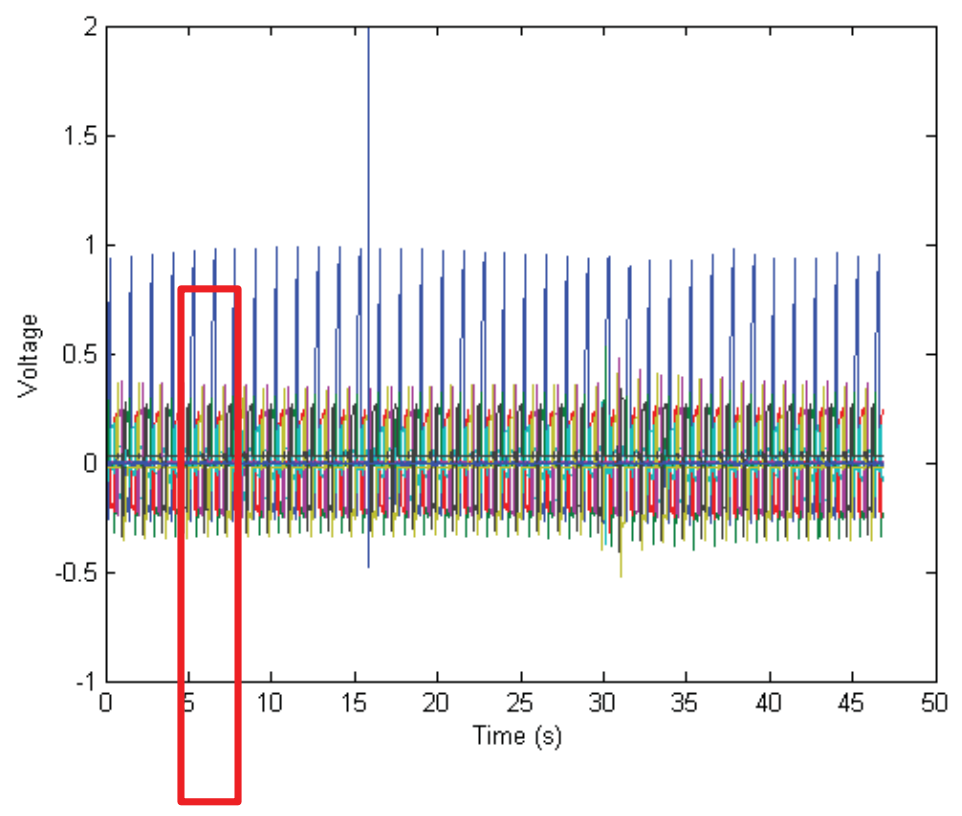

Figure 3-4 Typical raw measured data

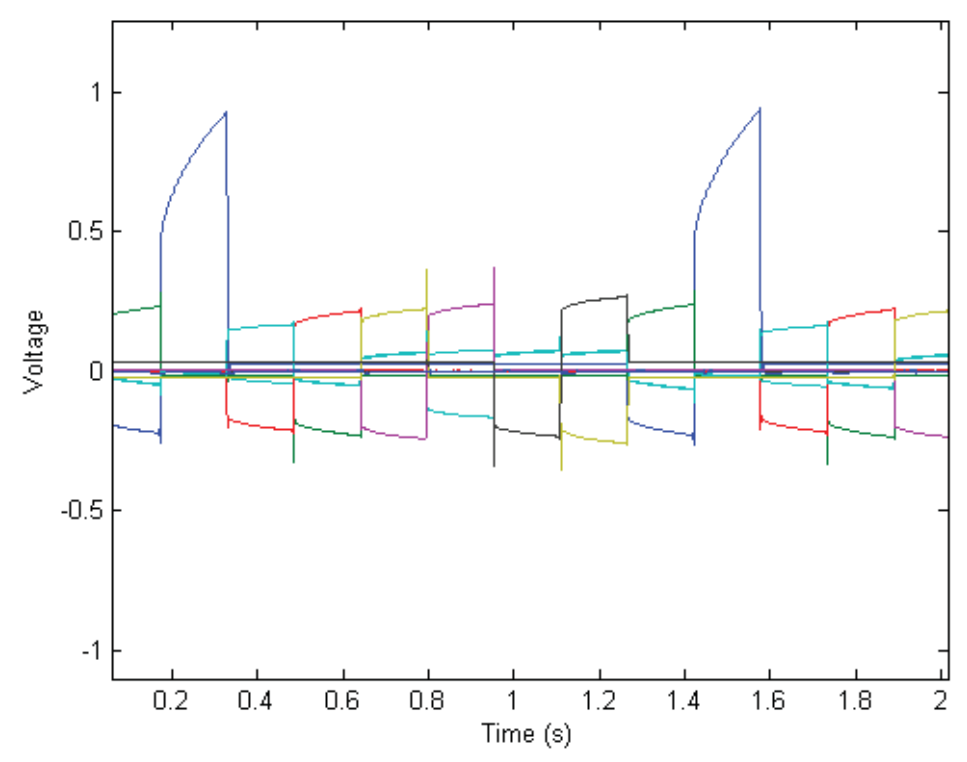

Figure 3-5 Zoomed section from Figure 3-4 


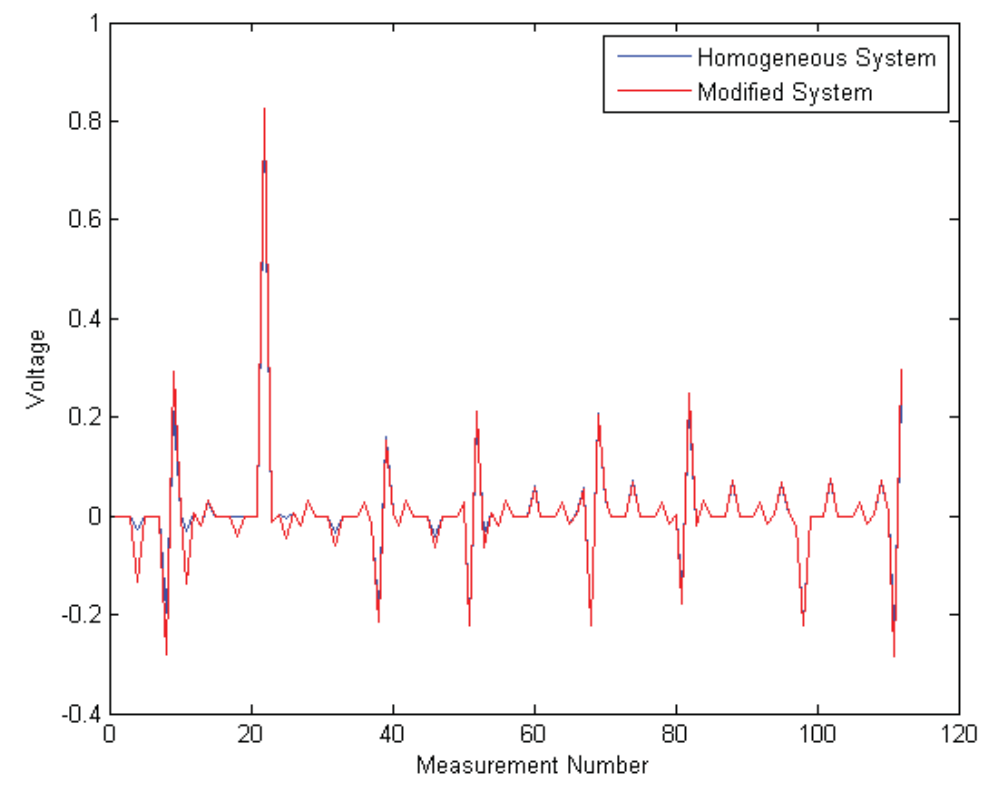

Figure 3-6 Processed data from typical run

\subsection{Proof of Concept}

As a proof of concept, a 4" diameter PVC pipe that was 30" long was used as an initial prototype for testing. Insulated wires ran into the pipe with exposed ends were situated along one side and used to measure voltage. A pair of wires that were centrally located at each end were used to supply a DC voltage. A wooden block was used as a resistive inhomogeneity and moved down and up in the pipe. By measuring voltage between adjacent electrodes as a function of time, it was possible to identify the vertical position of the wooden block. A plot of the voltage potentials between adjacent electrodes can be seen in Figure 3-7. With the success of this test, the decision was made to move forward on the main project. 


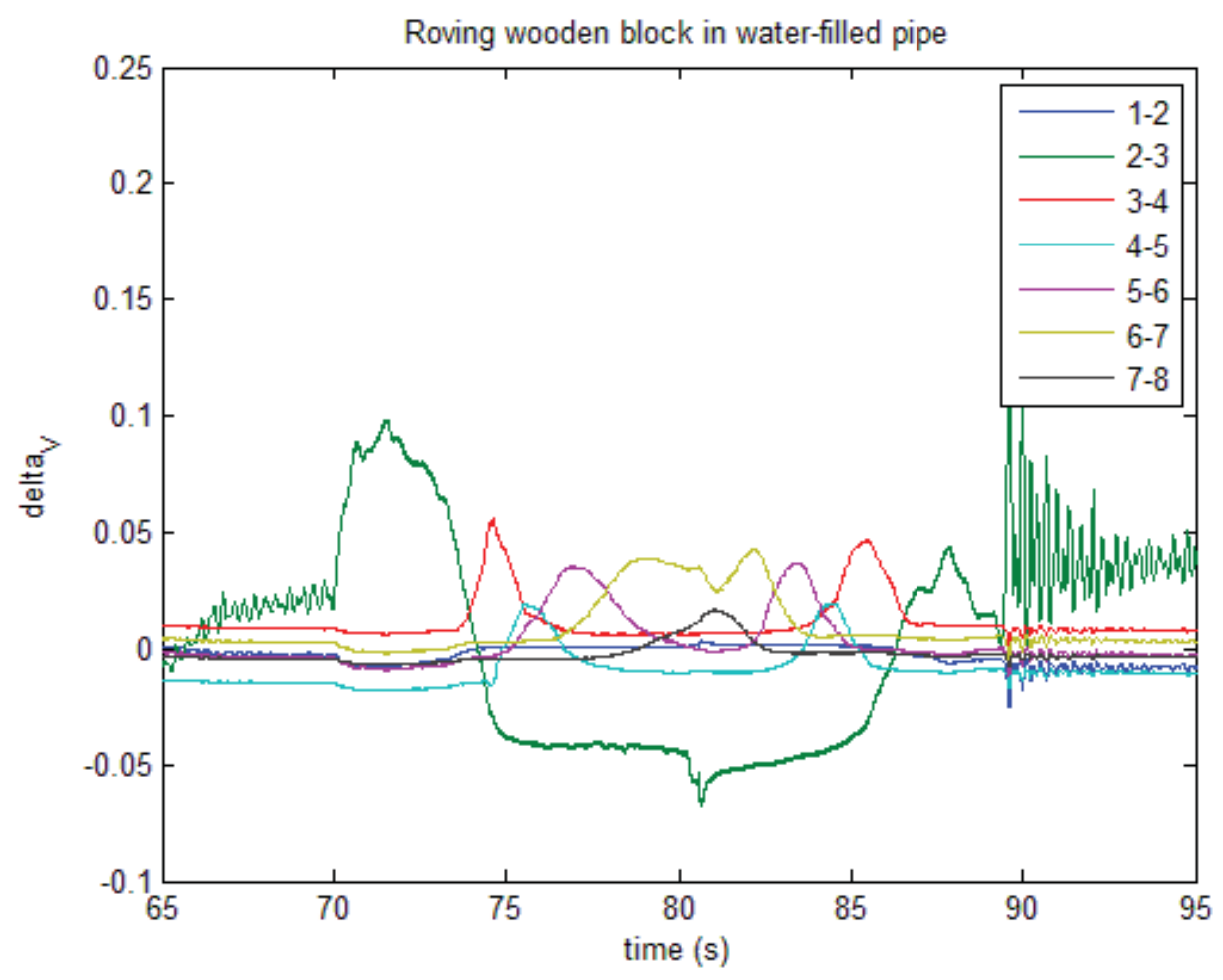

Figure 3-7 Voltage potentials between electrodes from proof of concept test

\subsection{Tests performed}

The focus of this project was to analyze fractures within ballistics gel. To create the fractures, two methods were used. The first was to fire a .22 caliber rifle into the gel. This created a dynamic fracture event where the air gap formed will be of a much higher resistivity and created an observable contrast. A high speed camera was used to record video footage during the test to provide test validation.

The second method used an injected fluid to create a fracture within the ballistics gel. The injected fluid used was salt water, which provided a contrast of lower resistivity than the gel. Dye was added to the salt water to provide a visual identification of the fracture so that the test data could be validated. 


\subsubsection{Preparation of Ballistic Gelatin}

The ballistic gelatin preparation procedure was based on the work of [16]. Gelatin powder was weighed out on a three beam balance and mixed with a measured volume of cold tap water to create a $10 \%$ by mass mixture of gelatin and water. The mixture of gelatin and water was allowed to sit for two hours while the gelatin hydrated. The mixture was then heated slowly on an electric stove while being stirred to prevent scorching. The mold was prepared by applying a silicone caulk bead to the seal to ensure that the gelatin would not leak out during testing. After the gelatin was poured into the mold, it was allowed to solidify over a period of 12 hours. At this point, warm water was used to warm the outside of the mold to melt the outside layer of the gelatin and release it from the mold. The sample was then put into a plastic bag to prevent it from drying out and stored at 45 degrees Fahrenheit until it was fractured. After testing, it was necessary to dispose of the sample within a day of testing because the ballistic gelatin provides an excellent medium for bacterial growth.

\subsubsection{Hydraulic Fracture Imaging}

A cylinder of ballistic gelatin was prepared and fitted with the electrode array. Data was recorded while it was in its homogeneous state. Green saline solution was loaded into a syringe and the syringe was inserted into a cylindrical gelatin sample in the space between electrodes 3,4,11, and 12. A picture of this can be seen in Figure 3-8. The entrance point of the syringe was sealed with duct tape and 25 milliliters of saline was injected, fracturing the gelatin. The resulting fracture can be seen in Figure 3-9 and extends from the space between electrodes 2 and 10 to the space between electrodes 5 
and 13. It can be seen here that after a few minutes, some of the saline dissolved the gelatin and the fracture extended on its own.

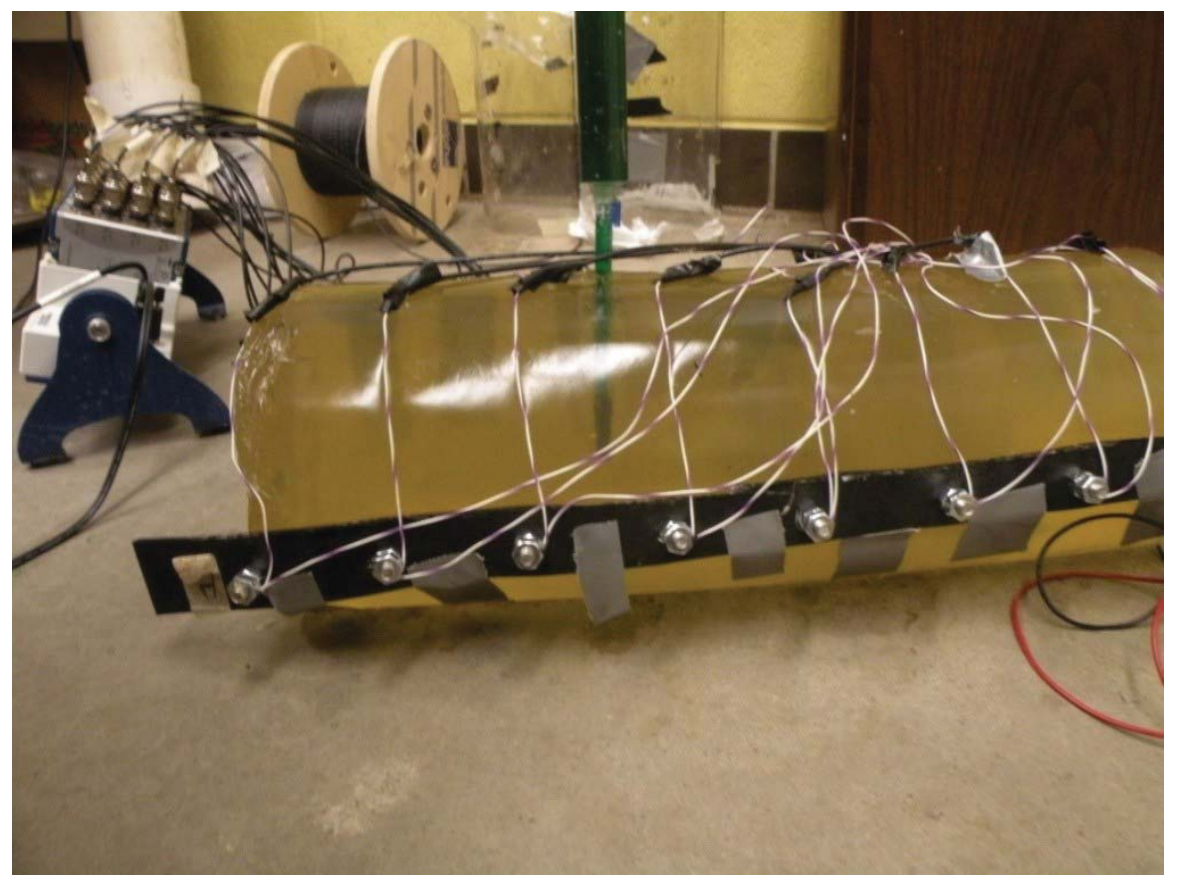

Figure 3-8 Syringe inserted in gelatin block with electrode array attached 


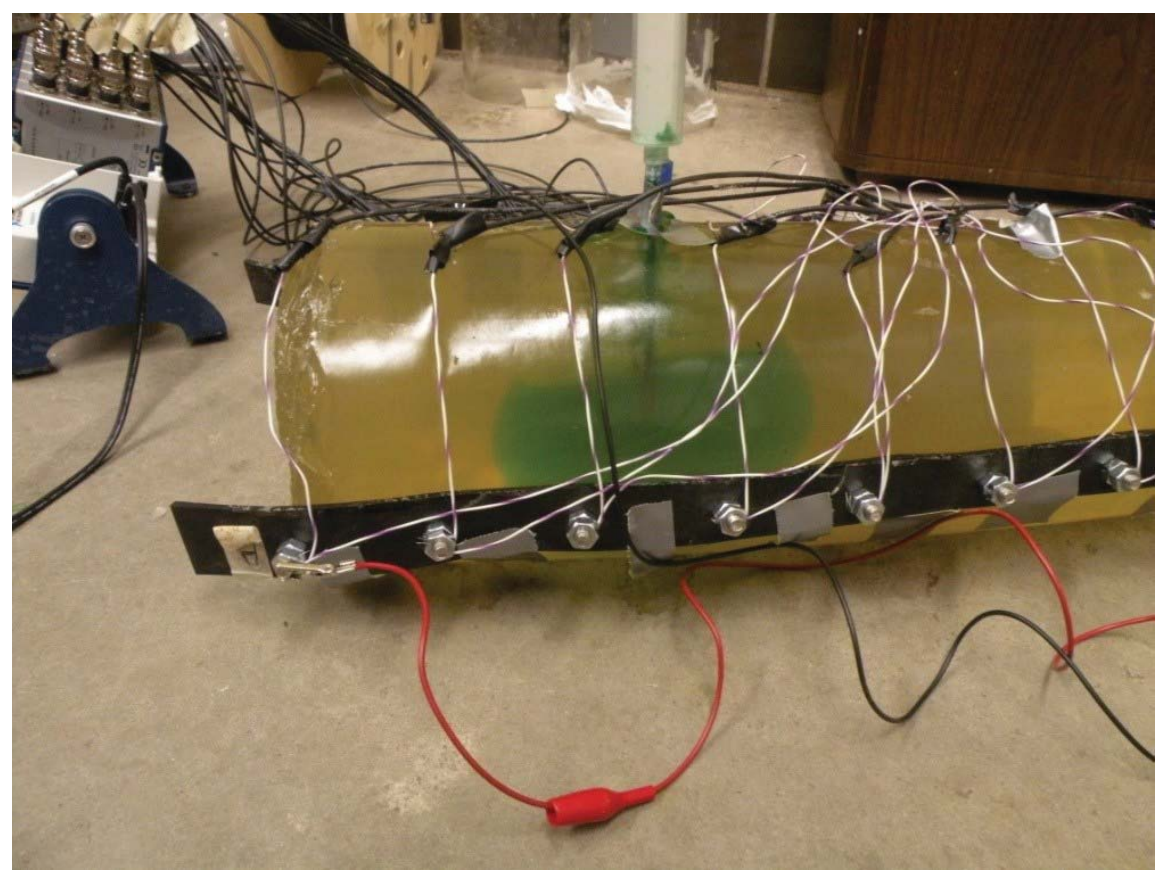

Figure 3-9 Fracture resulting from saline injection

Data was collected and processed using the method described in Section 3.5. A comparison matrix was developed with the One Step Gauss Newton method to determine appropriate hyperparameter and Jacobian background values, and is included in Table 3.1. Using this technique, a hyperparameter of 0.1 was selected and used for following inversions. A Jacobian background value of 0.1 was also selected to accompany the hyperparameter. 
Table 3-1 Hyperparameter and Jacobian background selection matrix

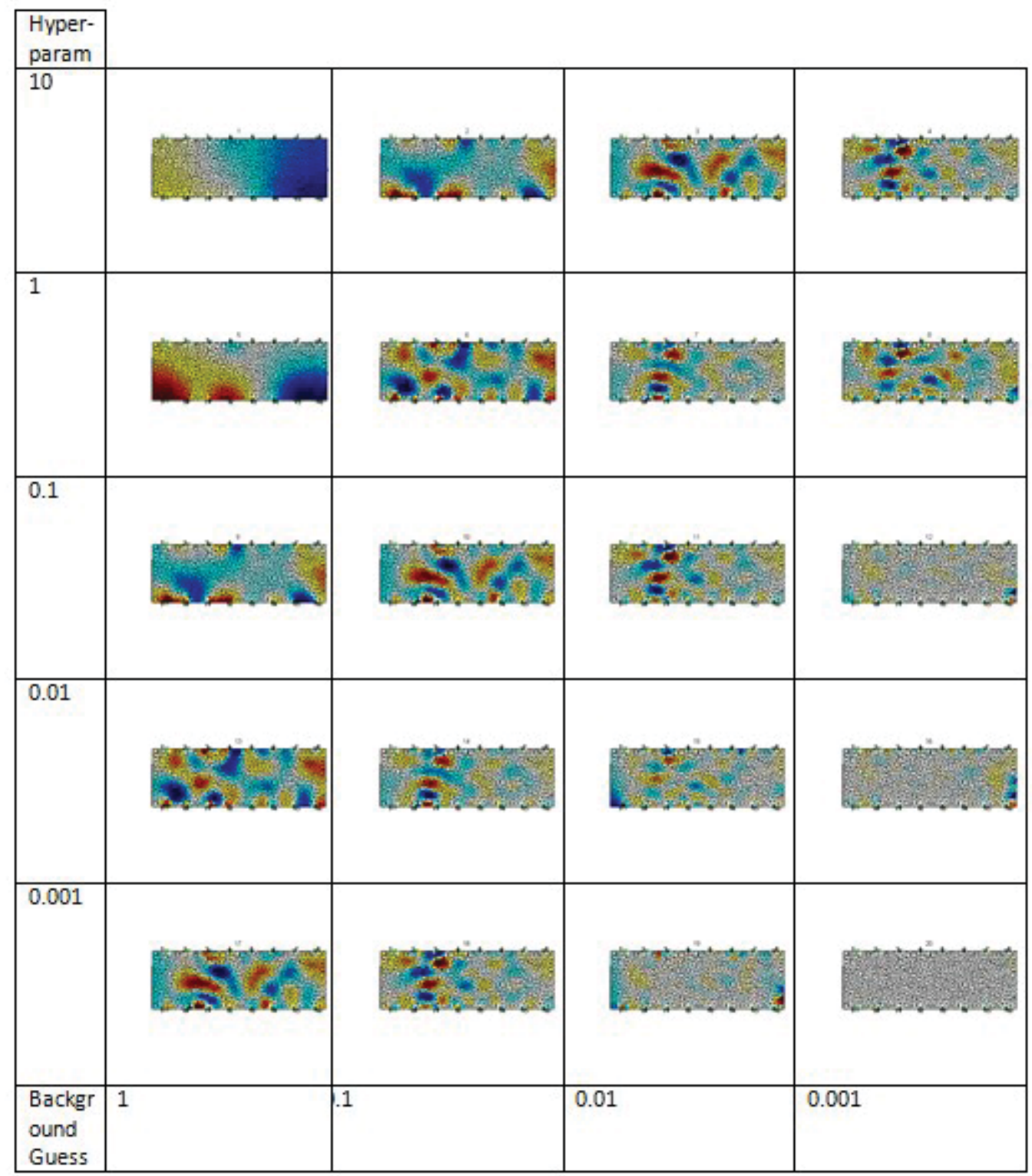

Four types of difference imaging inversion were performed, Truncated SVD (TSVD), Conjugate Gradient, Total Variation, and One Step Gauss Newton. These are included below as Figure 3-10, Figure 3-11, Figure 3-12, and Figure 3-13 respectively. The One 
Step Gauss Newton method was selected as the preferred inversion method for the rest of this section.

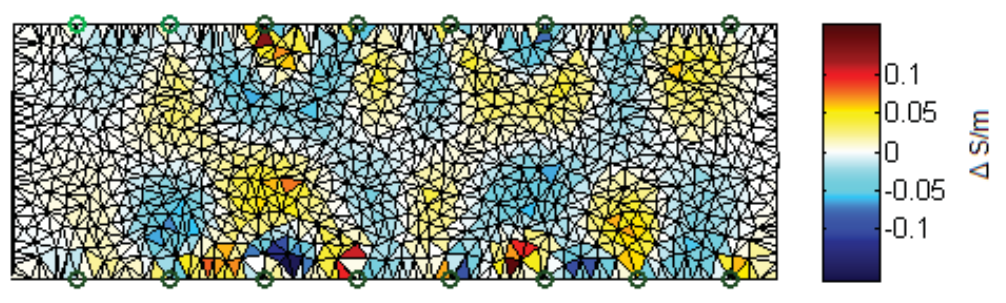

Figure 3-10 Difference image reconstructed with TSVD method 


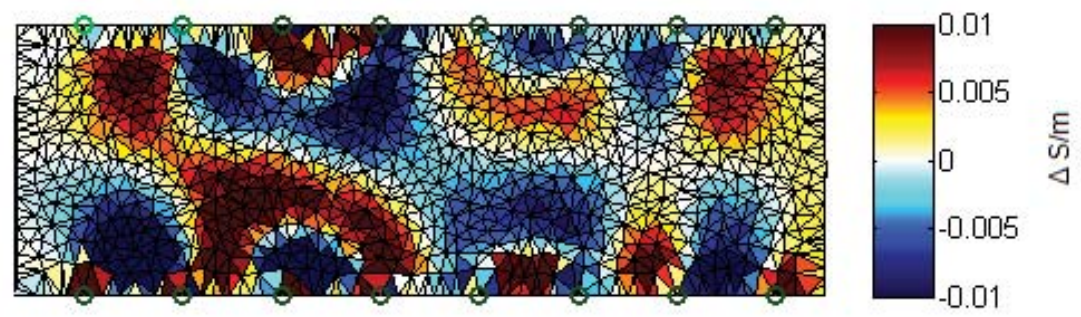

Figure 3-11 Difference image reconstructed with Conjugate Gradient method

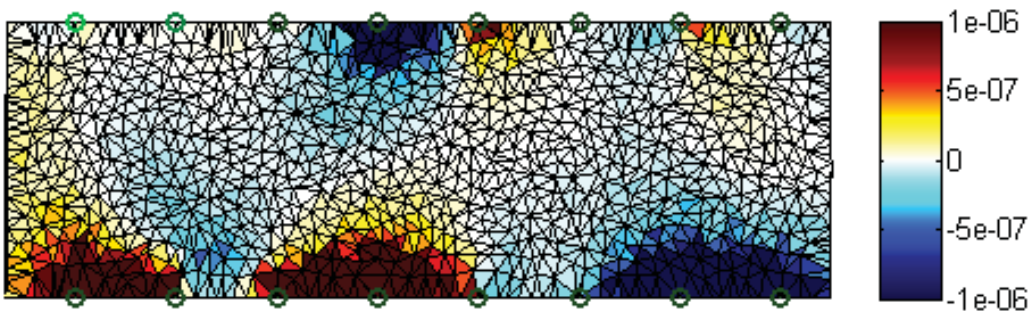

Figure 3-12 Difference image reconstructed using Total Variation method 


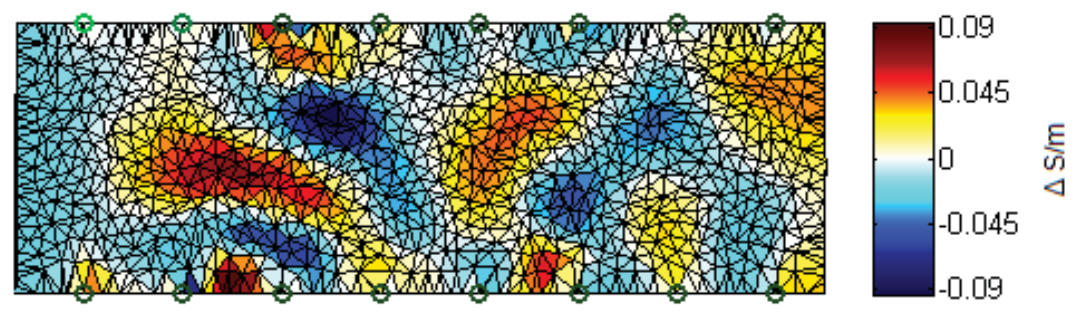

Figure 3-13 Difference image reconstructed with one step Gauss Newton method In an attempt to improve the quality of the resulting inverted cross-section, electrode measurement error correction based on the method of [37] was applied to the data. This method requires the operator to select electrodes that are providing erroneous measurements. For this test, electrode 10 was selected due to the large decrease in conductivity seen next to it. Only minor differences were noticed in the resulting reconstruction, as can be seen in Figure 3-14. 


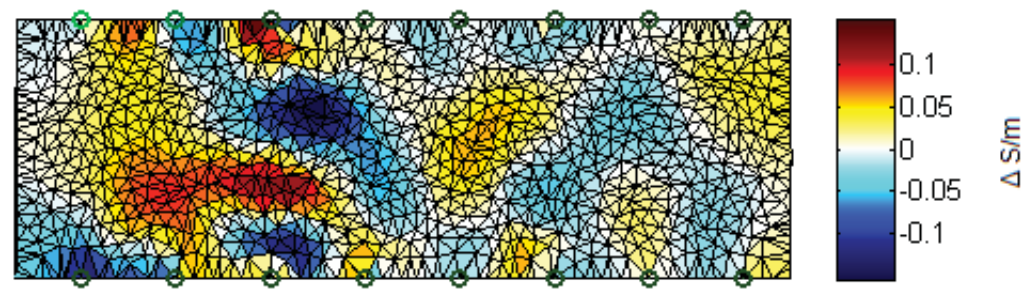

Figure 3-14 Difference image reconstructed accounting for error on electrode 10

A significant difference was noticed on the absolute solving of the homogeneous data, which is apparent when comparing the absolute images with and without electrode error correction. This can be seen in Figure 3-15 and Figure 3-16. From this, it was determined that the electrode error correction was not necessary and probably was counter-productive for this data set. Code for an automatic method for detecting and accounting for electrode error based on [38] was available within EIDORS, but not usable on this data as the method requires reciprocity between measurements and stimulus pairs. 


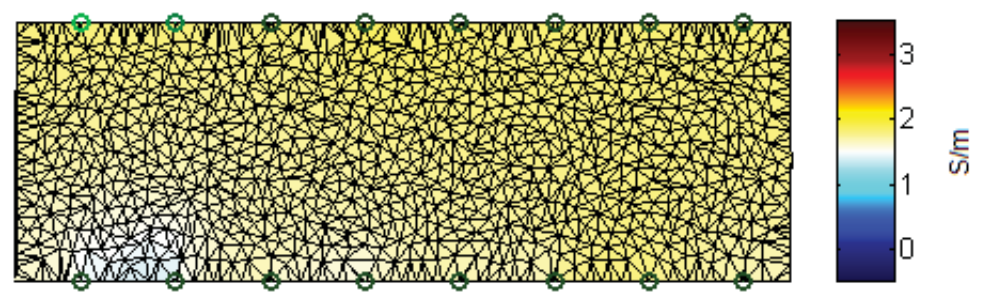

Figure 3-15 Static image reconstruction of homogeneous model

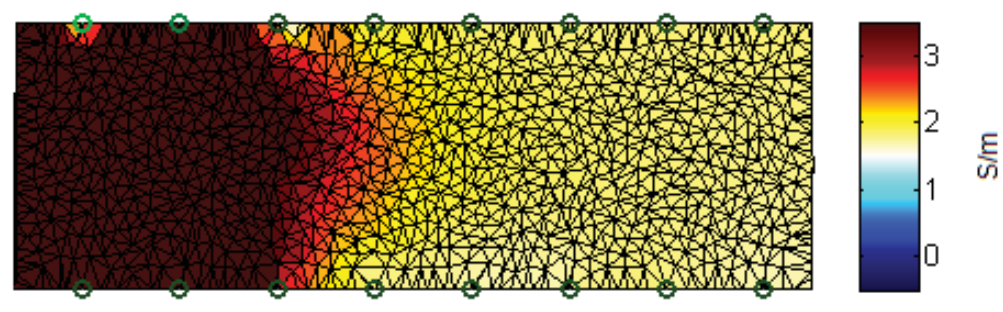

Figure 3-16 Static image reconstruction of homogeneous model with electrode error correction 
The results were validated in two ways. First, the sample was cut open at the electrode plane and the cross-section was photographed. The unfractured section of the sample was cut off first to conserve material and allow for another test. Without this end, the sample was weak and fell apart before the camera was readied. The held together cross-section can be seen in Figure 3-17 and the fallen apart cross section can be seen in Figure 3-18. In Figure 3-17, the approximate location of the electrodes has been marked as well as the syringe entrance hole. In Figure 3-18, the syringe entrance hole has been marked and the extent of the formed cavity is traced for clarity.

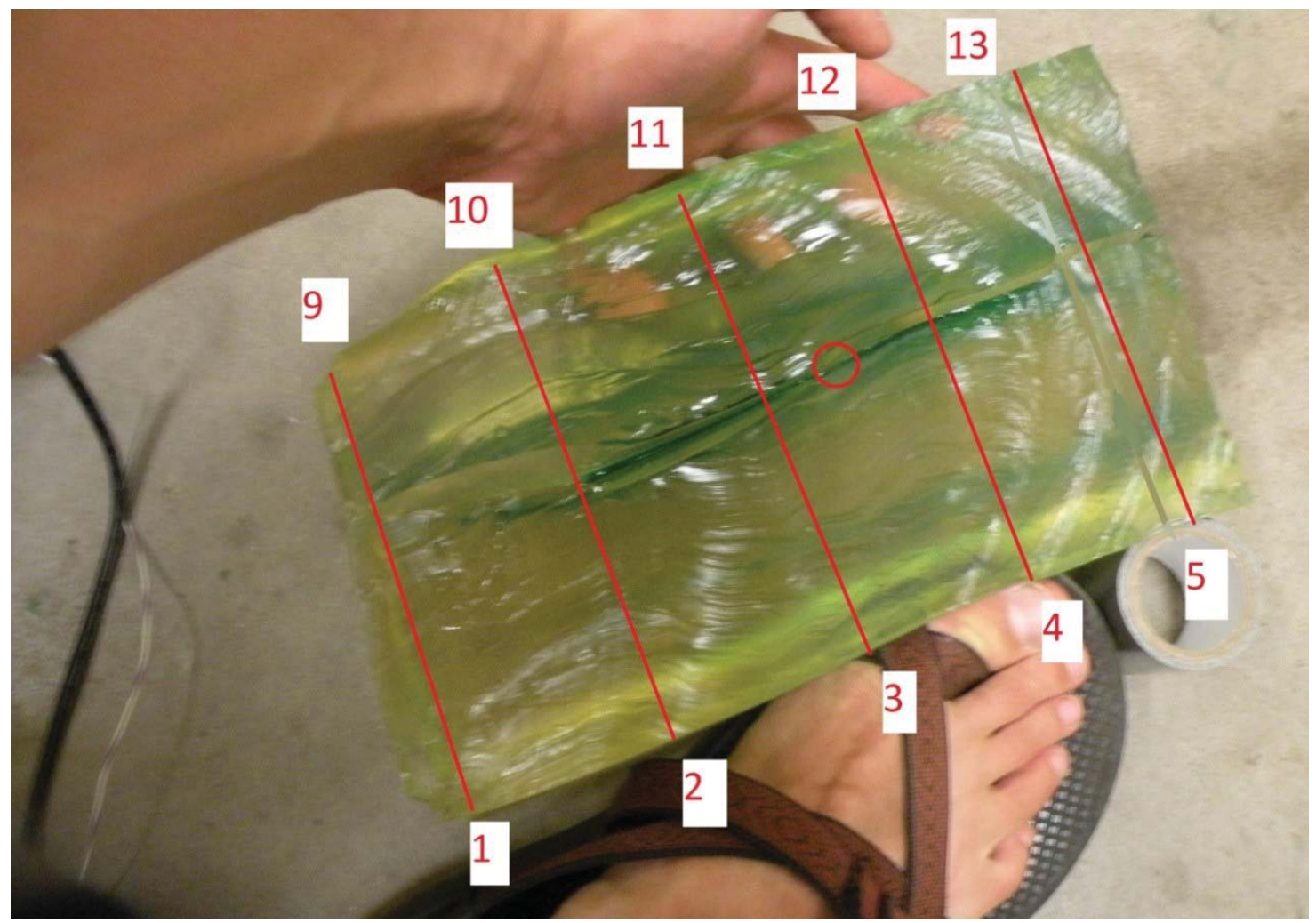

Figure 3-17 Cross-section of fracture along electrode plane 


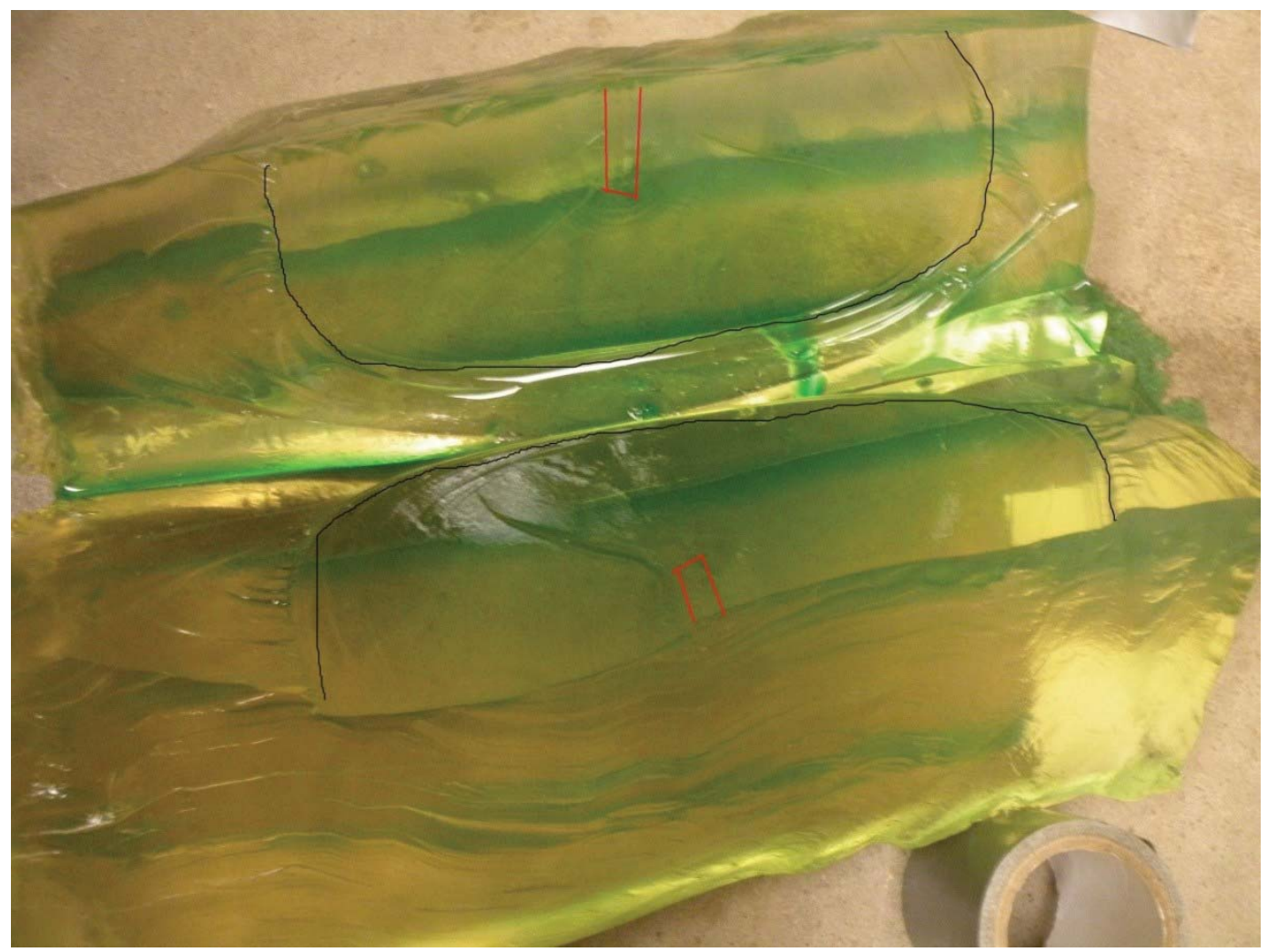

Figure 3-18 Split open fracture of gelatin

The second method of validation is intended to validate that the application of voltage instead of current allows for a representative cross-section. As mentioned earlier, a process based on [36] was used. Without measuring current during the test, it was not possible to ensure that the desired current was applied and so it was estimated based on preliminary sample measurements. To check whether the resistivity distributions determined with the voltage sources were valid, a set of data was taken with a current source. The alligator clips used for this can be seen in Figure 3-8. The resulting inverted cross-section can be seen in Figure 3-19. It can be seen that although amplitudes are different from Figure 3-13, the patterns are the same. Data from the unmodified sample were used to construct a static image of the absolute resistivity, which is included as 
Figure 3-20. The low conductivity near electrode 9 indicates that there may not have been a good connection between the gelatin and the electrode.

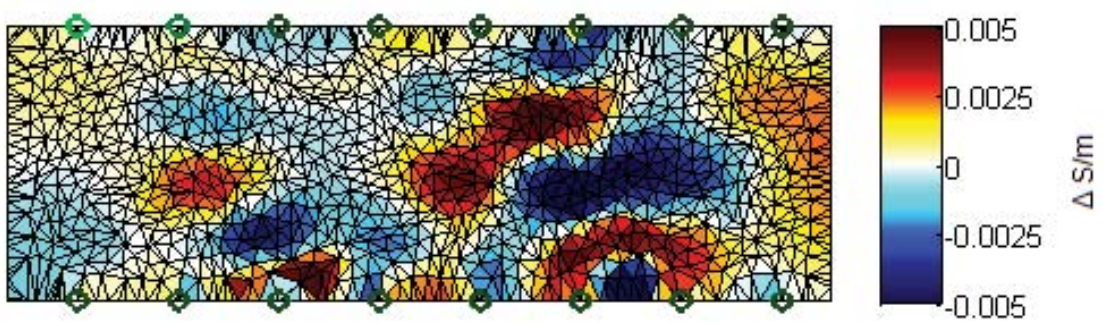

Figure 3-19 Difference image reconstructed with current source as stimulus 


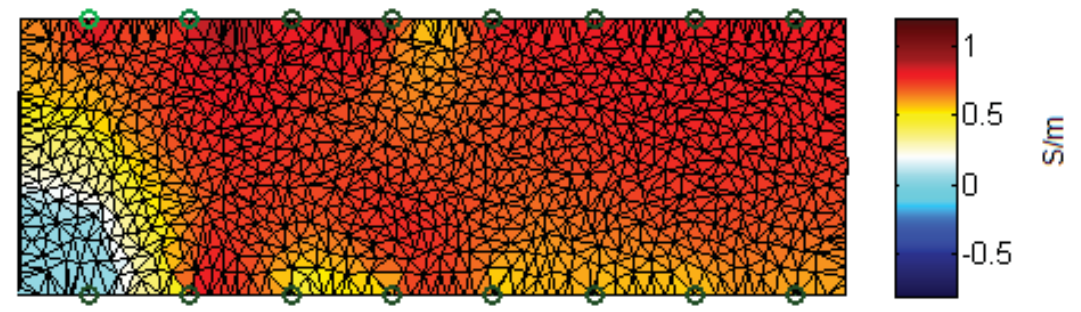

Figure 3-20 Static image of unmodified sample reconstructed with current source as stimulus

\subsubsection{Discussion of Hydraulic Fracture Imaging Results}

It was shown in the previous sections that the One Step Gauss Newton method provides reasonable looking results for a section of increased conductivity. This was validated by examining cross sections of the fracture itself, where it could be seen that the fracture spans the distance approximated in the reconstructed resistivity section. It was also found that the assumptions made that allowed the use of voltage sources instead of current sources do not allow accurate conductivity values to be determined, but can still show the distribution of conductivities. 


\subsubsection{Temporary Cavity Fracture Imaging}

In order to use a rifle safely, the temporary cavity imaging tests were performed at Michigan Technological University's rifle range located in the Student Development Complex and outdoors at the Keweenaw Research Center. A picture from the test at the $\mathrm{KRC}$ can be seen below in Figure 3-21. Not pictured is the extra lighting that was used to improve image quality or the large sand berm located beyond the target (behind the view of the photograph.) The image has also been flipped horizontally so that orientation of the gelatin sample matches the orientation of the inverted images shown in the rest of the section.

A high speed camera was focused onto a cylindrical block of ballistics gelatin that was resting on a pallet. One person operated the camera, another operated the DAQ, and a third fired the rifle. Two representative video sequences from the high speed camera can be seen in Figure 3-22 and Figure 3-23, with the ring and linear electrode arrays, respectively. The terminal ballistic sequence described in Section 1.4 can be seen in both of these images, particularly the growth and collapse of the temporary cavity. 


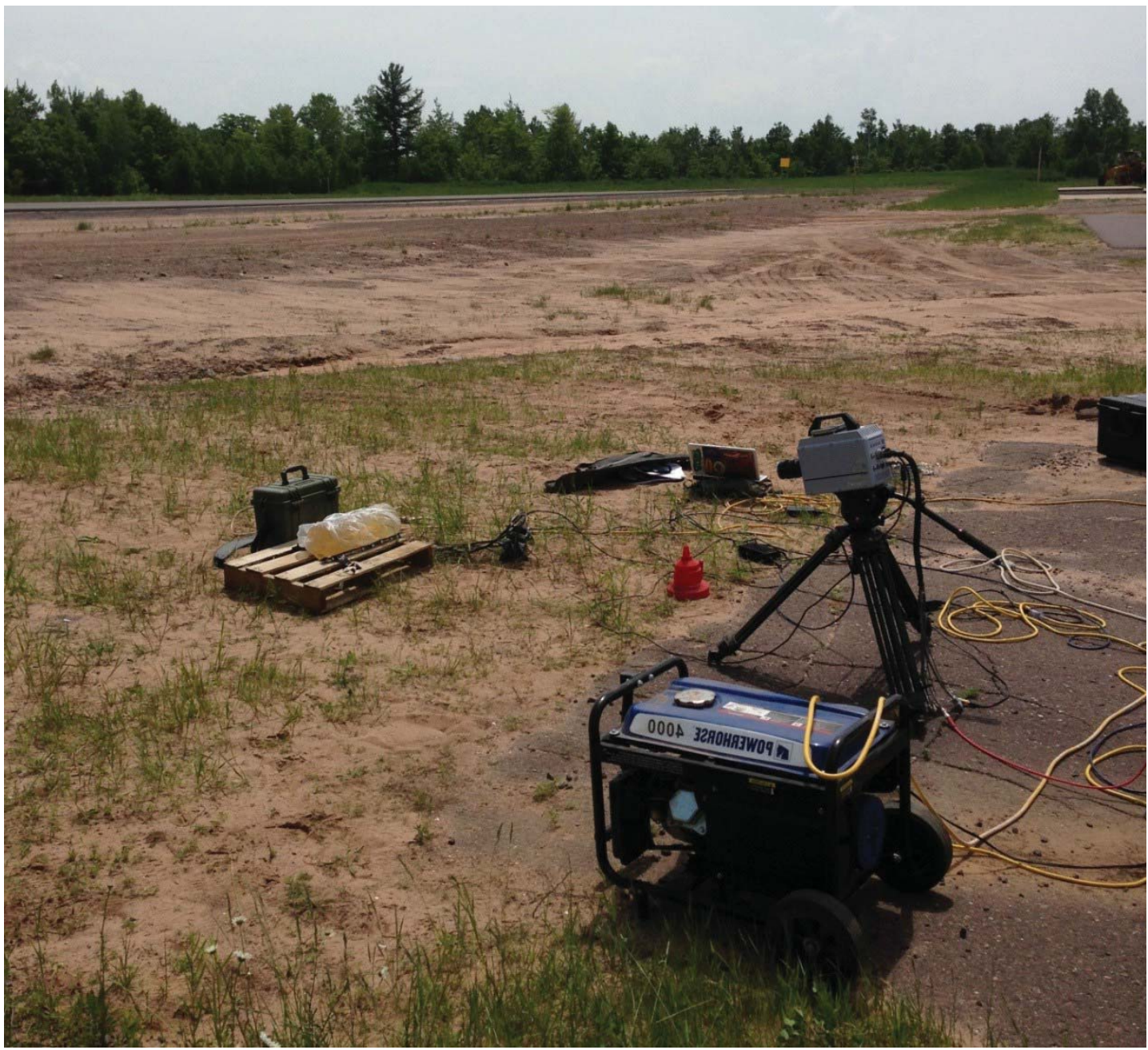

Figure 3-21 Test setup for temporary cavity imaging 


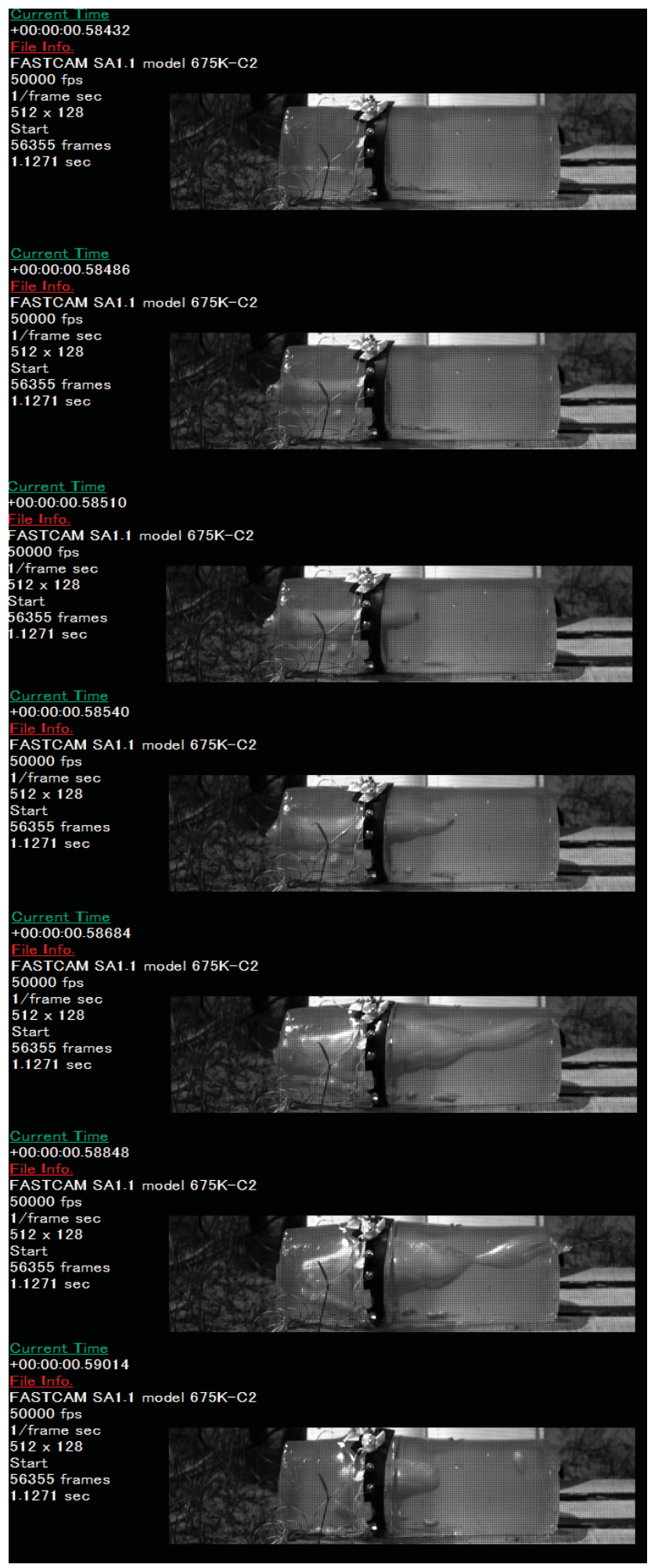

Figure 3-22 Representative video sequence of temporary cavity test with ring array 


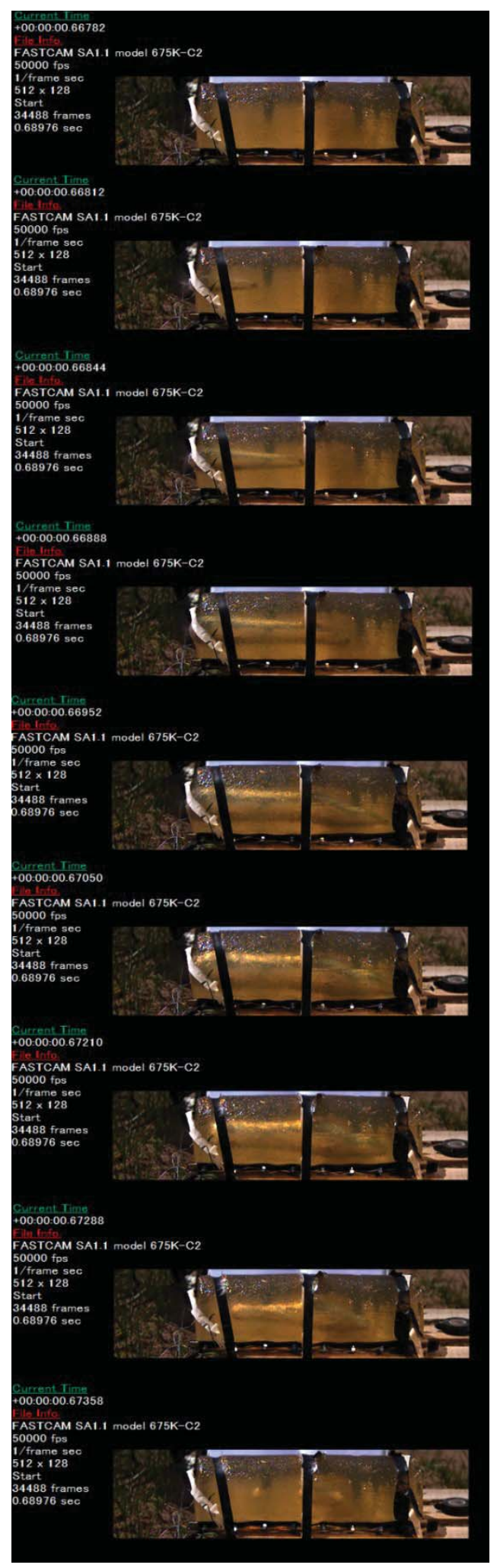

Figure 3-23 Representative video sequence of temporary cavity test with linear array (shot 11) 
In order to correlate the high speed video footage to the EIT imaging, one input channel on the DAQ was used to record a trigger input from the camera. Although the DAQ was not set up to trigger off of this input, it recorded the input and provided a time reference for when camera recording started. An example of this is shown in Figure 3-24, where the blue trace that spikes above two volts is the trigger channel indicating that the camera has started recording. The change in voltage measurements can be seen as well, if one looks closely near the $6.5 \times 10^{4}$ sample. The video footage was then analyzed to determine the time offset between the beginning of camera footage and the impact of the bullet. It was found that there was a good correlation between time offset of the changing inverted frames and timestamp of bullet impact on camera for shot 11. Unfortunately camera data was lost for shot 10 and only one data point was available to validate the EIT data.

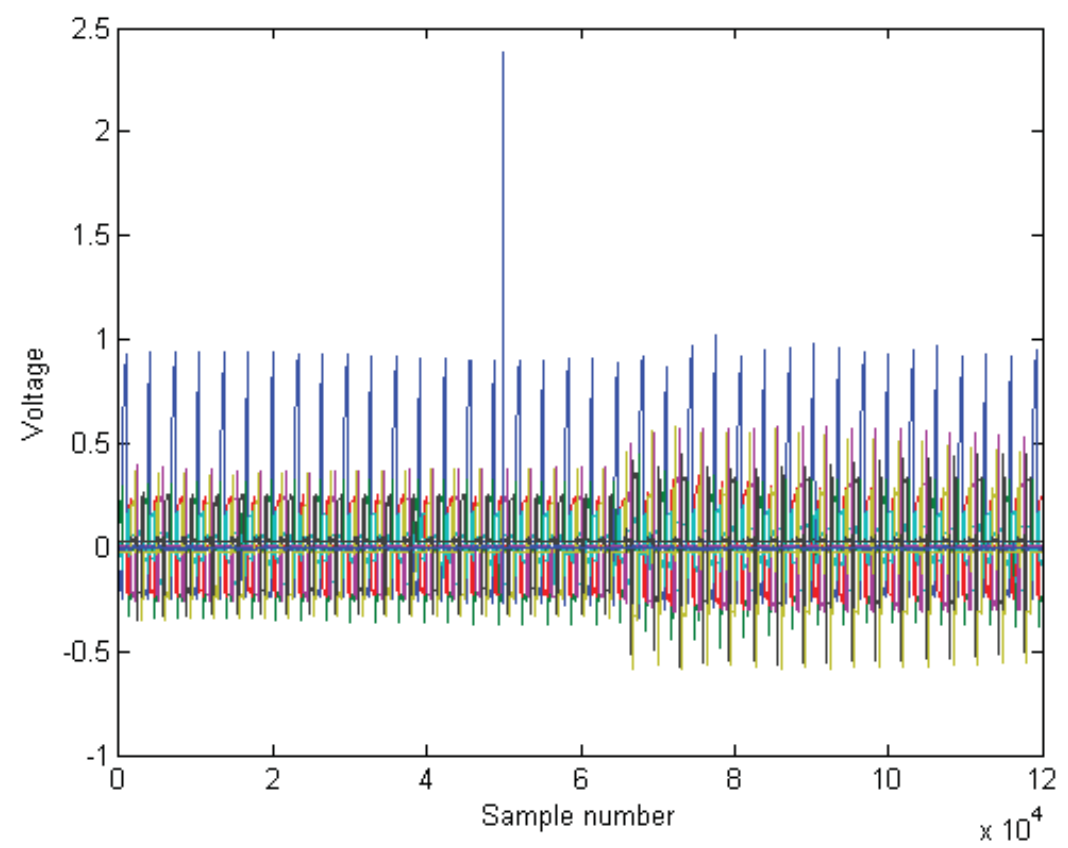

Figure 3-24 Time history from shot 11 from all input channels 
Data from the time history was split into frames, as explained in Section 3.2.2, and difference data was calculated by comparing the measurements to the measurements taken from the unmodified gelatin block. This time series of measurements was then inverted using a One Step Gauss Newton reconstruction method and the series of reconstructed images were plotted together to observe the change in conductivities with time. The image time series for shot 10 can be seen in Figure 3-25 and the series for shot 11 can be seen in Figure 3-26. Detailed views of the frames capturing cavities and the ones before and after the cavity event can be seen in Figure 3-27 through Figure 3-33. The slow time resolution of 0.125 seconds used for this step means that a single frame eclipses the entire temporary cavity event shown in Figure 3-23.

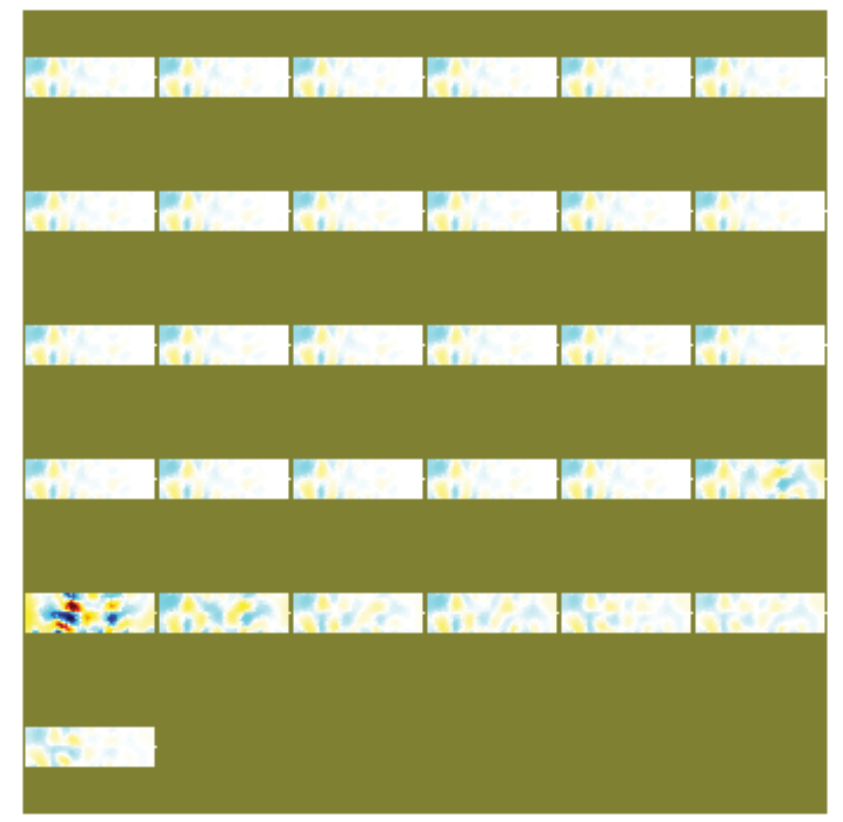

Figure 3-25 Difference image time series for shot 10 


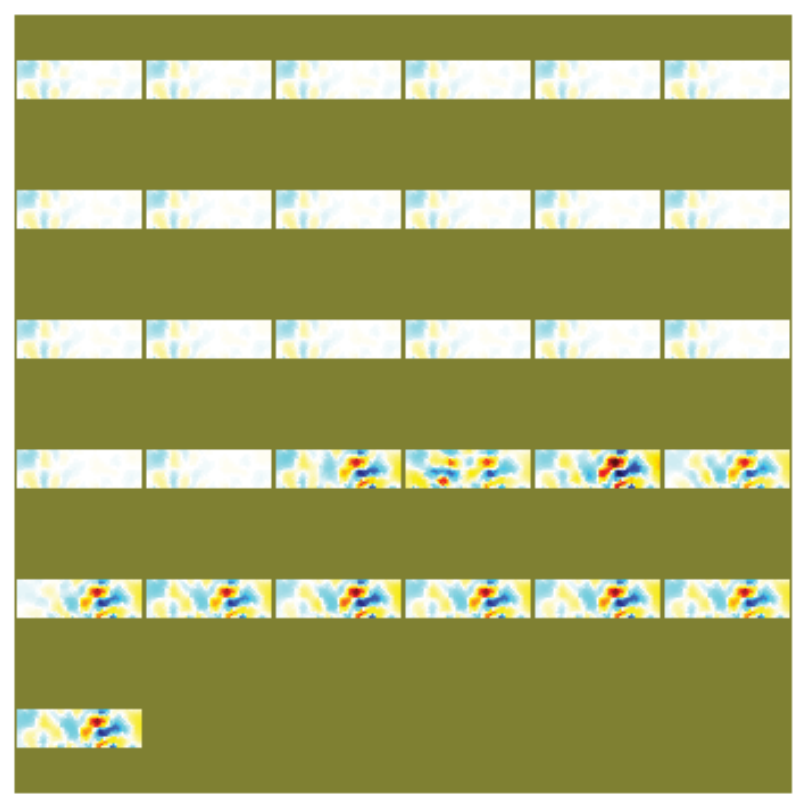

Figure 3-26

Difference image time series for shot 11

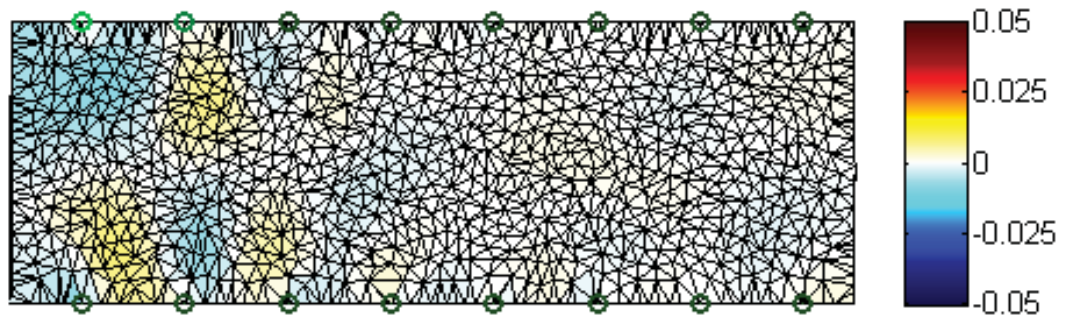

Figure 3-27 Frame 20 of Shot 11 


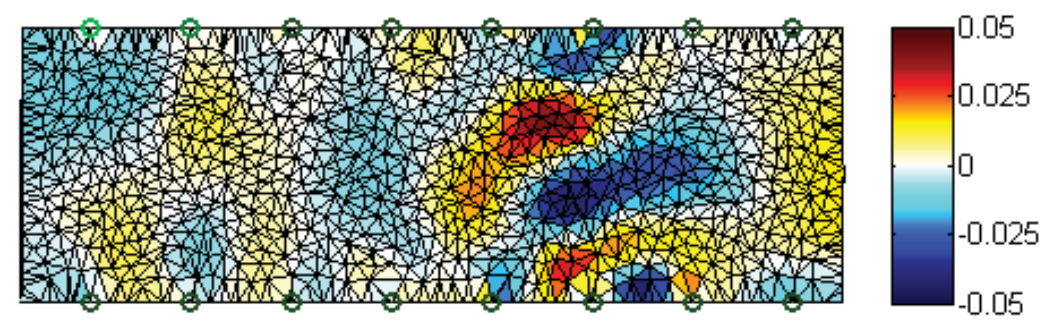

Figure 3-28 Frame 21 of Shot 11

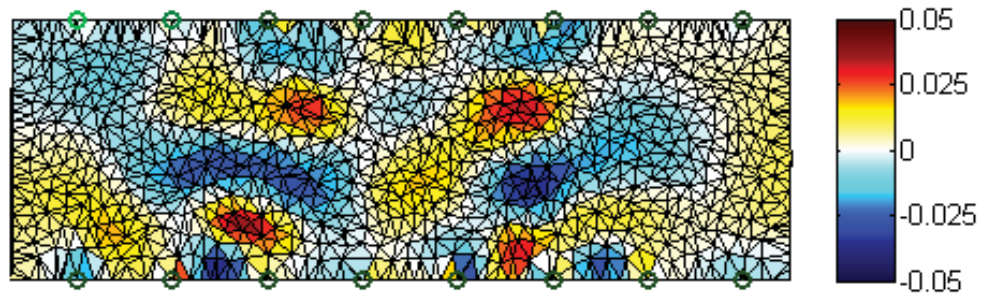

Figure 3-29 Frame 22 of Shot 11 


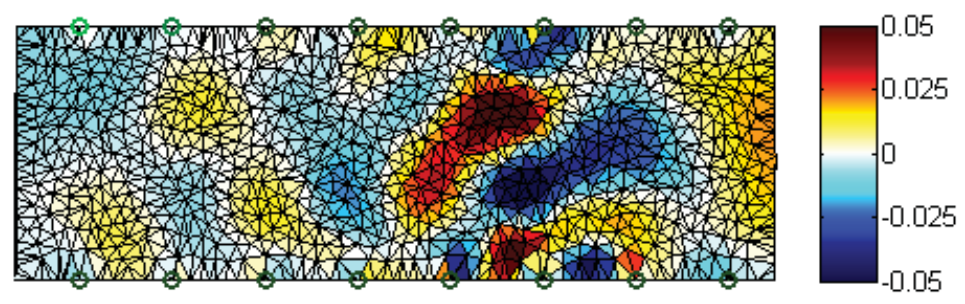

Figure 3-30 Frame 23 of Shot 11

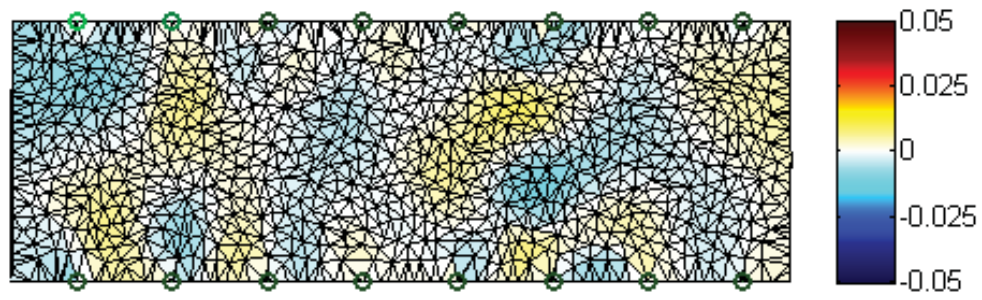

Figure 3-31 Frame 24 from Shot 10 


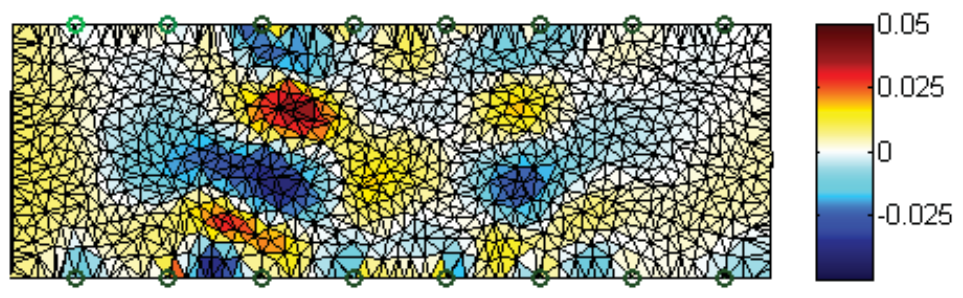

Figure 3-32 Frame 25 from Shot 10

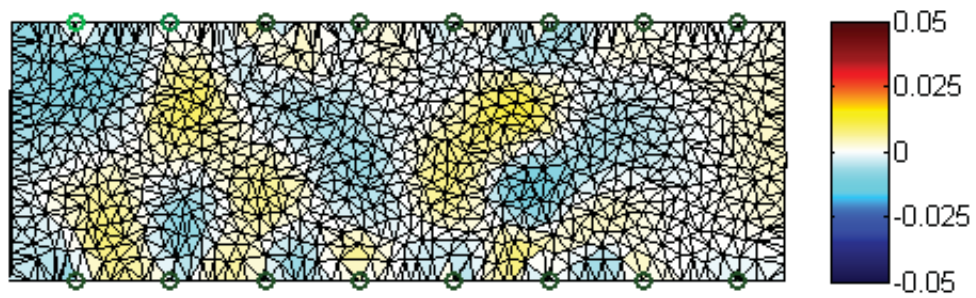

Figure 3-33 Frame 26 from Shot 10 


\subsubsection{Discussion of Temporary Cavity Imaging Results}

The images above demonstrate that this technique is very good at detecting changes on a system level. The images generated from the inversion process appear to include centrally located decreases in conductivity, which is indicative of a cavity. There are areas of high conductivity immediately adjacent to the areas of low conductivity. These could be explained as either being areas of compressed gelatin or being reconstruction errors, where the reconstruction algorithm generates a resistivity distribution that oscillates spatially while decaying towards zero.

It is worth noting that when preparing the image series for shot 10 and shot $11, a$ measurement was immediately before each shot to count as the "homogeneous" measurement of the difference image, where each other measurement would be compared to it.

\subsection{Simulation of Circular Array}

Due to an issue with the construction of the circular array, electrical shorts were introduced into the wiring, making the data from the physical circular array useless. To provide some insight to the capability of the circular array, a simulation was performed where the stimulation/measurement series was simulated on the finite element model (performing a forward problem) and then inverted. The One-Step Gauss Newton method used for the linear array was also used for simulation of the ring array. The finite element mesh used for the simulations can be seen in Figure 3-34. First the Jacobians were calculated using this mesh with a uniform conductivity applied, a sample of which is 
included as Figure 3-35. Then three separate geometries of inhomogeneities were tested, the first of which is in Figure 3-37.

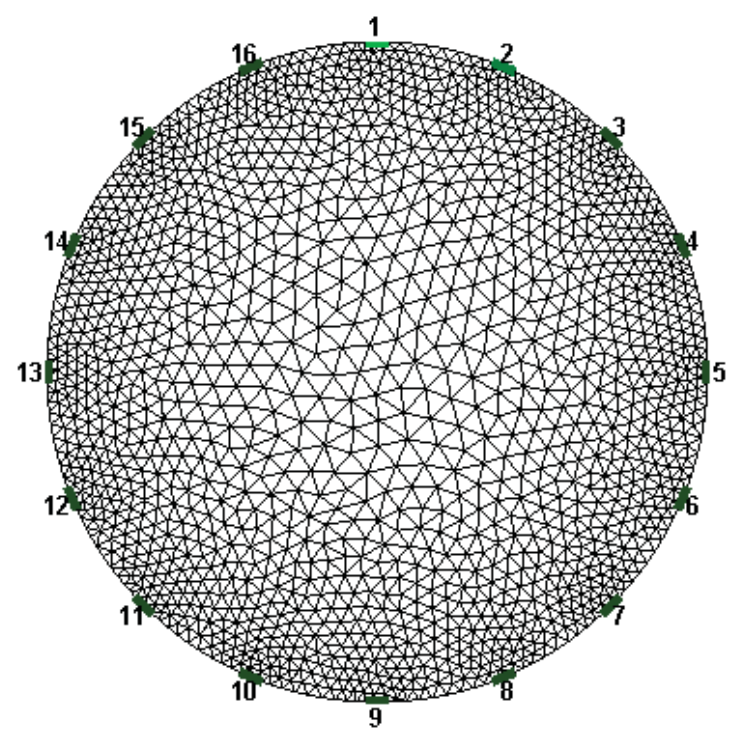

Figure 3-34 Finite Element Mesh used for simulations

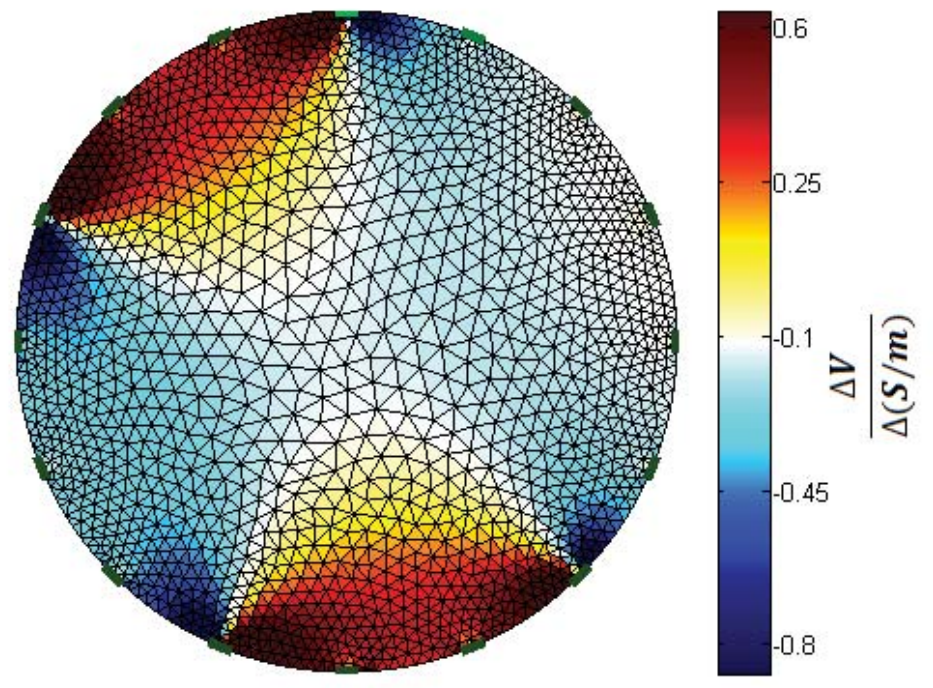

Figure 3-35 Sample Jacobian 


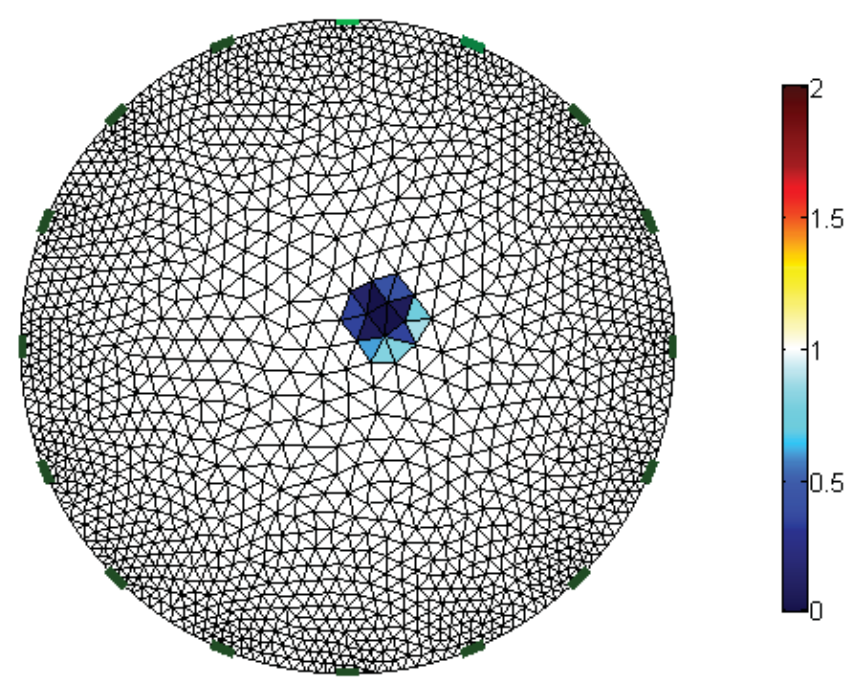

Figure 3-36 Non Homogeneous FEM 1

The first non-homogeneous finite element mesh used included a highly resistive circular inclusion to simulate a small air cavity or plastic straw. A test was then simulated and the resulting measurements were inverted using the same method and parameters used in the linear array case (difference method using the homogenous case as a reference and the One-Step Gauss Newton reconstruction method with the same hyperparameter of 0.1.) The resulting inversion can be seen in Figure 3-37. There are two things important to note in this image. The first is the enlargement of the inclusion and the second is the ringing effect around it. Although there was no increases in conductivity, there is an apparent increase in conductivity immediately adjacent to the resistive inclusion. 


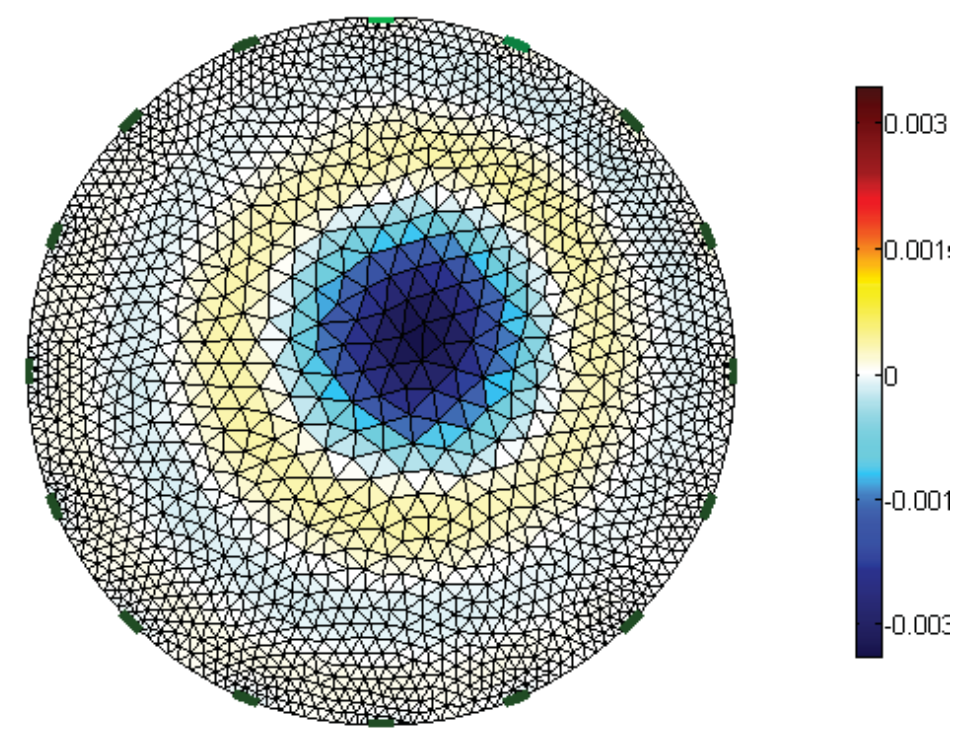

Figure 3-37 Resulting Inversion from Non Homogeneous FEM 1

The second geometry tested was a thin, conductive fracture. The finite element model used can be seen in Figure 3-38. Again, the same procedure was performed and the resulting inversion can be seen in Figure 3-39. Here the same issues as seen in the first model are still present. There is an enlargement of the inclusion, which blurs the actual fracture-like geometry initially simulated. Again, there is a ringing effect around the inclusion, where there appears to be a region of a mild increase in conductivity surrounding the inclusion. 


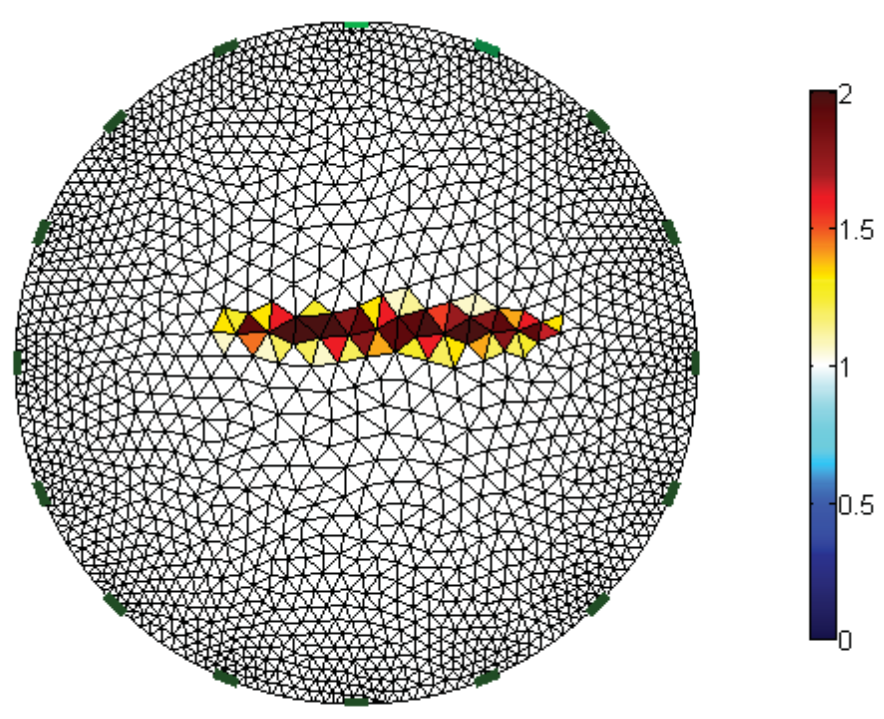

Figure 3-38 Non Homogeneous FEM 2

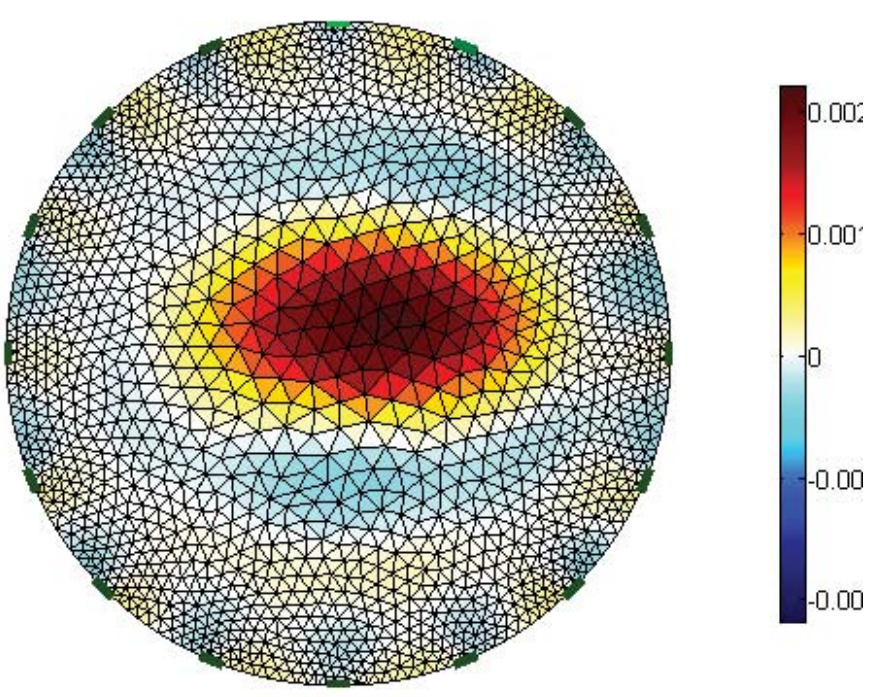

Figure 3-39 Resulting Inversion from Non Homogeneous FEM 2 
The third finite element model tested combined a thin conductive fracture with a resistive circular inclusion at the center of the fracture. The idea was to simulate the saline fracture with the straw at the center of it. The model used can be seen in Figure 3-40. The same procedure as used in the previous models was repeated and the resulting inversion can be seen in Figure 3-41. The same issues arise again, with one additional issue. The resistive inclusion is completely unseen and its existence can only be inferred by the strange shape seen in the reconstruction.

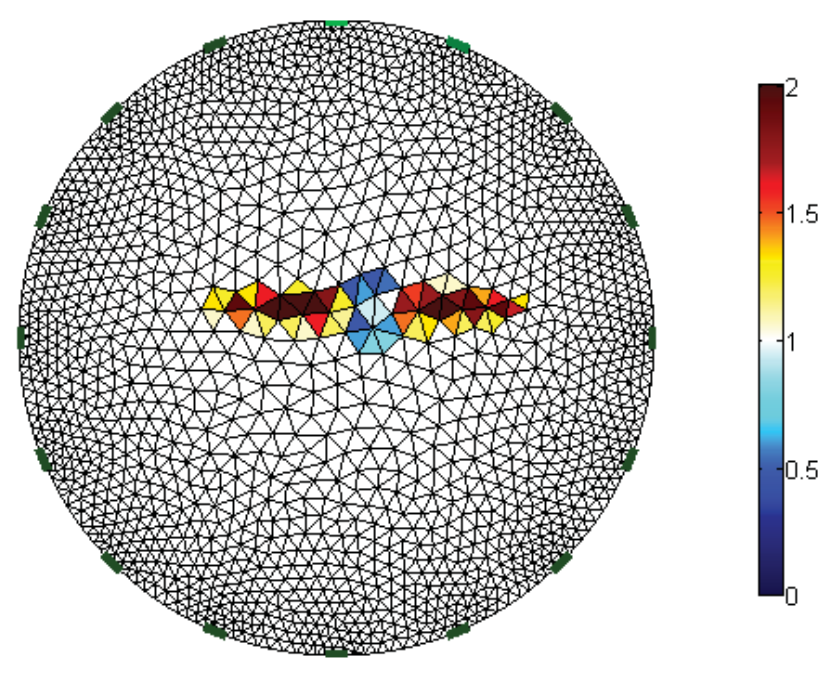

Figure 3-40 Non Homogeneous FEM 3 


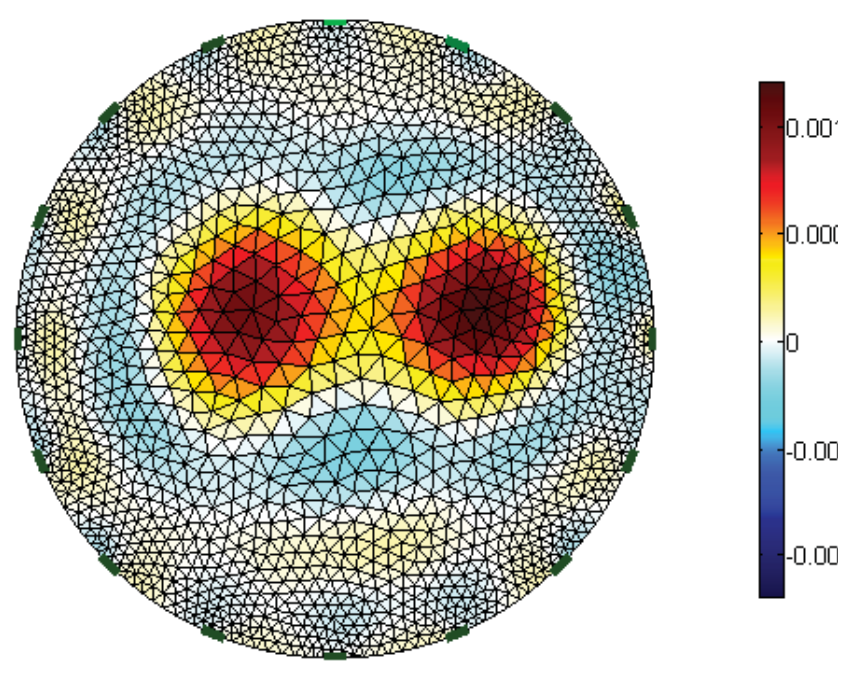

Figure 3-41 Resulting Inversion from Non Homogeneous FEM 3

To further understand the inverted images from the experimental portion of the work, an effort was made to make the simulation more realistic and noise was added to the simulated measurements. Noise at three different signal to noise ratios (SNR) was simulated using MATLAB's additive white Gaussian noise function: $80 \mathrm{~dB}, 70 \mathrm{~dB}$, and 60 $\mathrm{dB}$. The resulting inversions can be seen in Figure 3-42 through Figure 3-44. The quality of the reconstruction can be seen to degrade with the decrease in SNR. At the 60 $\mathrm{dB}$ point, it is no longer clear that the reconstructed image is representative of the original finite element model. 


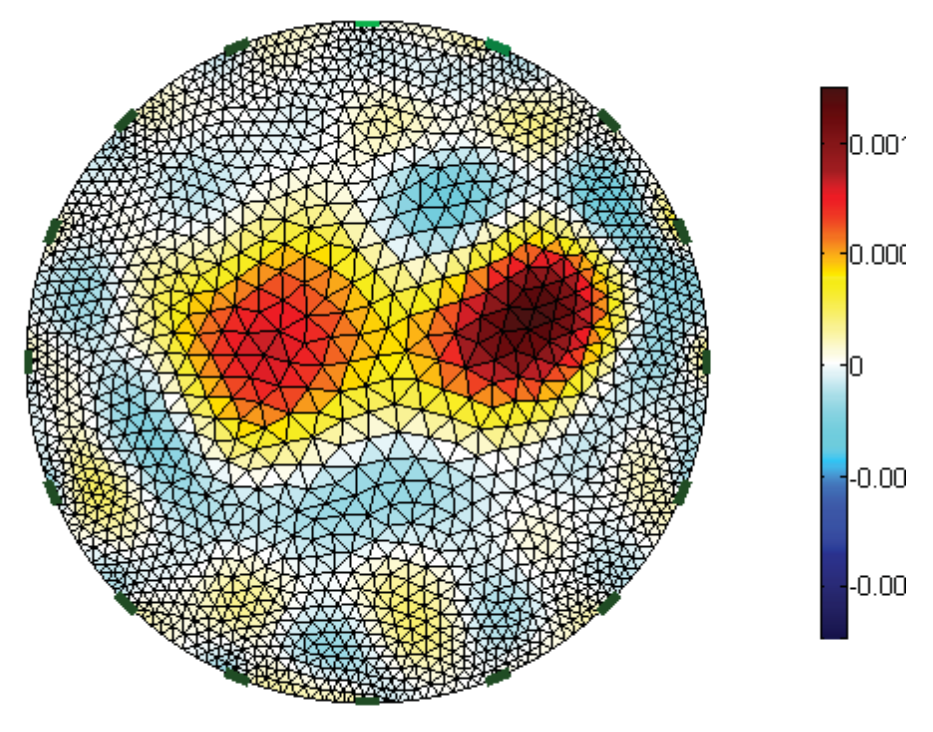

Figure 3-42 Resulting Inversion from Non Homogeneous FEM 3 with 80 dB SNR

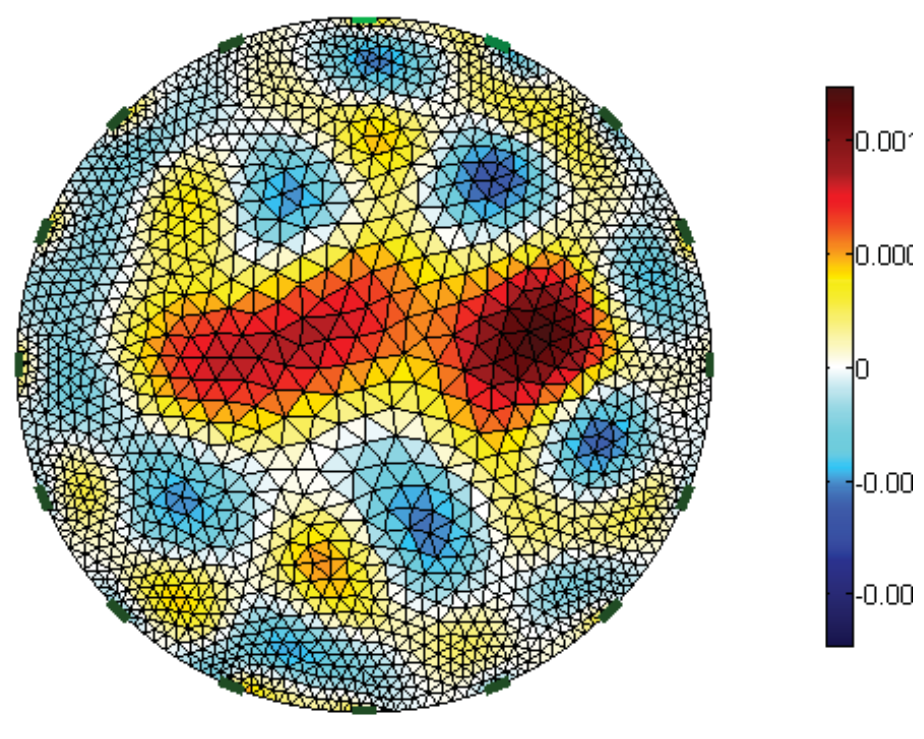

Figure 3-43 Resulting Inversion from Non Homogeneous FEM 3 with 70 dB SNR 


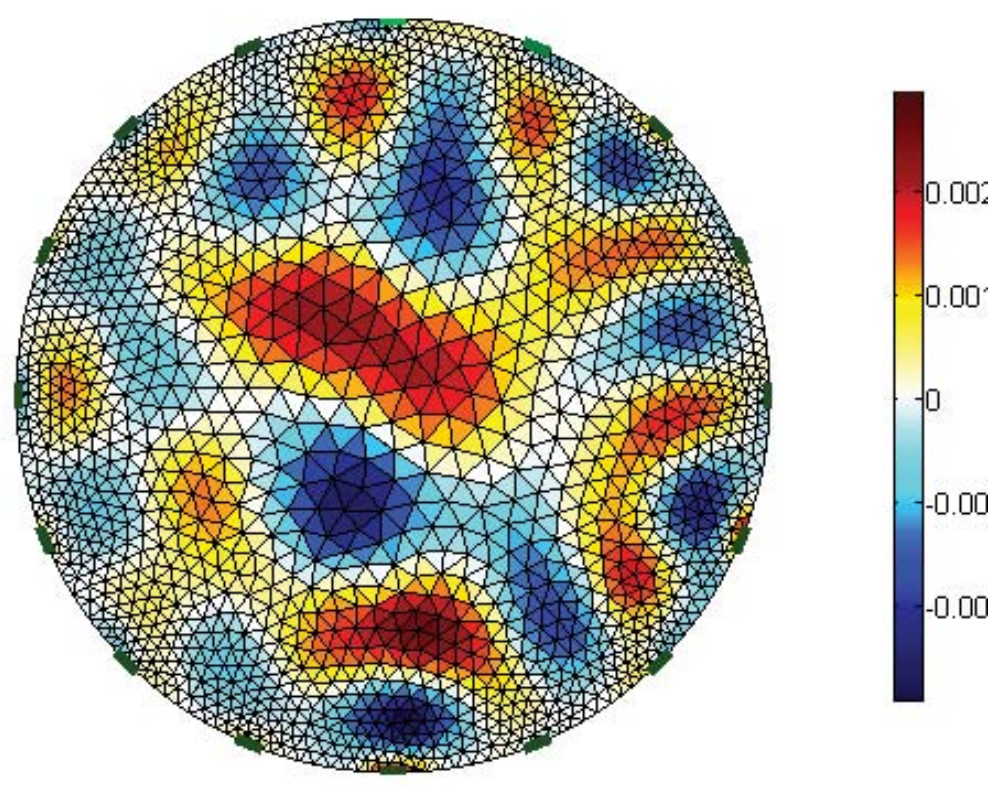

Figure 3-44 Resulting Inversion from Non Homogeneous FEM 3 with 60 dB SNR 


\subsection{Sensitivity Analysis}

To better understand the sensitivity of the technique, a sensitivity analysis was performed. The absolute value of all calculated Jacobians for a given geometry were summed and plotted. The results of this for the linear and ring arrays can be seen in Figure 3-45 and Figure 3-46, respectively. It can be observed that in both cases, the areas near the surface are far more sensitive to changes than the interior of the system. This makes sense, when thinking back to the physics of the problem, because the current density will be greater near the surface. The asymmetry seen in Figure 3-45 is not as easily explained.

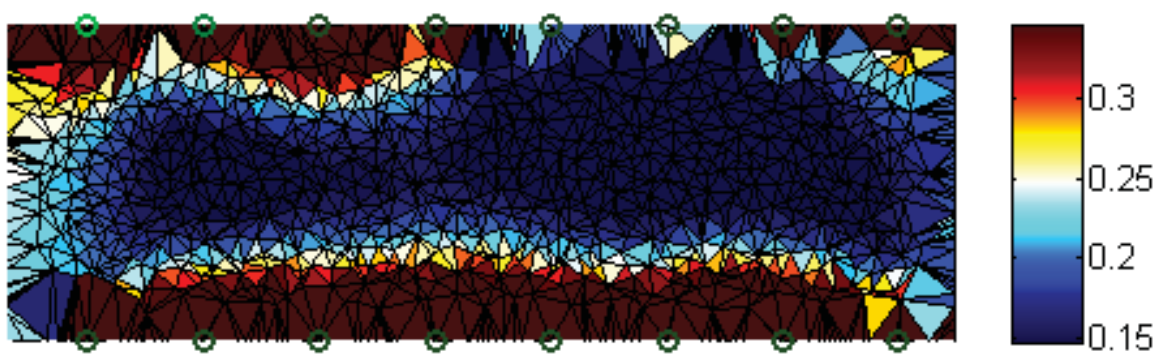

Figure 3-45 Sum of absolute values for all Jacobians for linear array geometry 


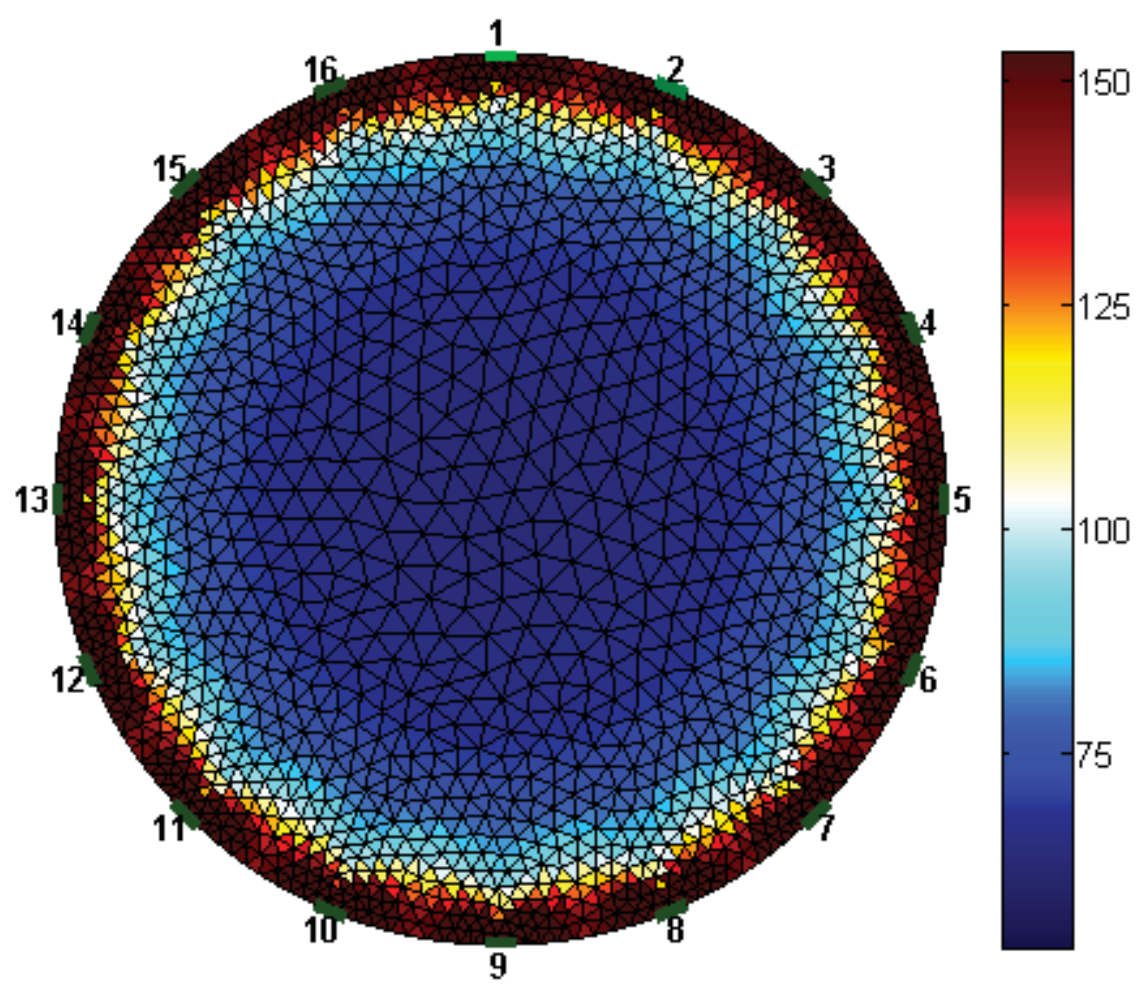

Figure 3-46 Sum of absolute values of all Jacobians for ring array geometry 


\section{Conclusions and Future Work}

\subsection{Conclusions}

It was demonstrated that EIT is a suitable technique for imaging fractures in ballistic gelatin, however many further steps must be taken to improve the resolution, both temporally and spatially. The use of more specialized equipment, including constant current drivers and faster DAQs will help improve both of these.

The simulation work performed is immensely helpful in helping understand the experimental results. By simulating a representative geometry, it was demonstrated that a ringing effect was common and exacerbated by noise. 


\subsection{Future Work}

\subsubsection{Future Work on Temporary Cavity Imaging}

Future work on imaging the terminal ballistics cavity should focus on improving the temporal resolution of the EIT. This could be achieved by using a faster DAQ and using AC currents that may not be as affected by the capacitive effects of the gelatin. Having a higher channel count would also allow the imaging to move into three dimensions by allowing the electrodes to be placed in an array format that is not planar. If electrodes were placed more densely, it would also improve the spatial resolution.

A correlation study between a simulation and experimental results would also help aid interpretation of the experimental results. This would also give the researcher an opportunity to attempt using reconstruction techniques that favor the sharp changes seen in fracture events.

\subsubsection{Future Work on Hydraulic Fracturing Imaging}

There are two major areas where future work could be performed on using EIT on hydraulic fractures. The first is to perform the calculations necessary to create a scale model and re-perform the experiment in a way that would be representative of a real-life test. This is a method that was popular before the advent of powerful computer simulations and gelatin has a history of being used as a medium for studying fracture propagation [39].

The second place future work is needed is in testing different inversion techniques in experimental crosswell resistivity tomography. Some work has already been performed on crosswell resistivity tomography, however the focus of the most thorough one was on natural fractures, including [40] and [41]. In the unconventional resources field, where 
hydraulic fracturing is currently seeing a lot of use, the fractures are man-made and usually in shales, a "soft" sedimentary rock. There has been some work on simulating the ability of crosswell resistivity tomography to image man-made shale fractures, including [37], that show promising results but experimental work will be needed to prove it out. 


\section{References}

1. Vauhkonen, M., Electrical impedance tomography and prior information. 1997.

2. Adler, A., P.O. Gaggero, and Y. Maimaitijiang, Adjacent stimulation and measurement patterns considered harmful. Physiological measurement, 2011. 32(7): p. 731.

3. Loke, M., Electrical imaging surveys for environmental and engineering studies. 2000 .

4. Bahrani, N., 21/2D Finite Element Method for Electrical Impedance Tomography Considering the Complete Electrode Model. 2012, Carleton University.

5. Samouëlian, A., et al., Electrical resistivity survey in soil science: a review. Soil and Tillage Research, 2005. 83(2): p. 173-193.

6. Krautblatter, M. and C. Hauck, Electrical resistivity tomography monitoring of permafrost in solid rock walls. Journal of Geophysical Research, 2007. 112(F2).

7. Holder, D.S., Electrical impedance tomography: methods, history and applications. 2005: Taylor \& Francis.

8. Murphy, S., et al., 3D electrical tomographic imaging using vertical arrays of electrodes. Measurement Science and Technology, 2006. 17(11): p. 3053.

9. Bolton, G.T., et al., Development of an electrical tomographic system for operation in a remote, acidic and radioactive environment. Chemical Engineering Journal, 2007. 130(2-3): p. 165-169.

10. Cheney, M., D. Isaacson, and J.C. Newell, Electrical impedance tomography. SIAM review, 1999. 41(1): p. 85-101.

11. Segesman, F., Well-logging method. Geophysics, 1980. 45(11): p. 1667-1684.

12. Martin, M., G.H. Murray, and W.J. Gillingham, Determination of the potential productivity of oil-bearing formations by resistivity measurements. Geophysics, 1938. 3(3): p. 258-272.

13. Nicholas, N. and J. Welsch, Ballistic gelatin. Institute for Non-Lethal Defense Technologies Report, Penn State Applied Research Laboratory, Happy Valley, PA, 2004.

14. Winter, J. and D. Shifler, The Material Properties of Gelatin Gels. 1975, DTIC Document.

15. Cronin, D. and C. Falzon. Dynamic characterization and simulation of ballistic gelatin. in 2009 SEM Conference \& Exposition on Experimental \& Applied Mechanics, June. 2009.

16. Jussila, J., Preparing ballistic gelatine-review and proposal for a standard method. Forensic science international, 2004. 141(2): p. 91-98.

17. Schyma, C.W.A., Colour contrast in ballistic gelatine. Forensic Science International, 2010. 197(1-3): p. 114-118.

18. Fackler, M.L. and J.A. Malinowski, The wound profile: A visual method for quantifying gunshot wound components. The Journal of trauma, 1985. 25(6): p. 522.

19. Hollerman, J., et al., Gunshot wounds: 1. Bullets, ballistics, and mechanisms of injury. American Journal of Roentgenology, 1990. 155(4): p. 685-690. 
20. FACKLER, M.L., R.F. BELLAMY, and J.A. MALINOWSKI, The wound profile: illustration of the missile-tissue interaction. The Journal of Trauma and Acute Care Surgery, 1988. 28(1): p. S21-S29.

21. Schyma, C. and B. Madea, Evaluation of the temporary cavity in ordnance gelatine. Forensic Science International, 2012. 214(1-3): p. 82-87.

22. Howard, G.C. and C.R. Fast, Hydraulic fracturing. NEW YORK, SOCIETY OF PETROLEUM ENGINEERS OF AIME, 1970. 210 P., 1970.

23. Hubbert, M.K. and D.G. Willis, Mechanics of Hydraulic Fracturing1. US Geological Survey, 1957. 210: p. 153-168.

24. Adler, A., T. Dai, and W.R. Lionheart, Temporal image reconstruction in electrical impedance tomography. Physiological measurement, 2007. 28(7): p. S1.

25. Johnson, T.J., Analysis of dynamic transmissibility as a feature for structural damage detection. 2002, Purdue University.

26. Borsic, A., Regularisation methods for imaging from electrical measurements. 2002, Oxford Brookes University.

27. Polydorides, N., Image reconstruction algorithms for soft-field tomography. 2002, University of Manchester: UMIST.

28. Adler, A. and W.R. Lionheart, Uses and abuses of EIDORS: an extensible software base for EIT. Physiol Meas, 2006. 27(5): p. S25-42.

29. Pidlisecky, A., E. Haber, and R. Knight, RESINVM3D: A 3D resistivity inversion package. Geophysics, 2007. 72(2): p. H1-H10.

30. Pidlisecky, A. and R. Knight, FW2 5D: A MATLAB 2.5-D electrical resistivity modeling code. Computers \& Geosciences, 2008. 34(12): p. 1645-1654.

31. Hansen, P.C., Rank-deficient and discrete ill-posed problems: numerical aspects of linear inversion. Vol. 4. 1987: Society for Industrial and Applied Mathematics.

32. Aster, R., B. Borchers, and C. Thurber, Parameter estimation and inverse problems: Elsevier Academic Press. Burlington, Massachusetts, 2005.

33. Lukaschewitsch, M., P. Maass, and M. Pidcock, Tikhonov regularization for electrical impedance tomography on unbounded domains. Inverse Problems, 2003. 19(3): p. 585.

34. Vauhkonen, M., et al., Tikhonov regularization and prior information in electrical impedance tomography. Medical Imaging, IEEE Transactions on, 1998. 17(2): p. 285-293.

35. Günther, T., C. Rücker, and K. Spitzer, Three-dimensional modelling and inversion of dc resistivity data incorporating topography - II. Inversion. Geophysical Journal International, 2006. 166(2): p. 506-517.

36. Hartov, A., et al., Using voltage sources as current drivers for electrical impedance tomography. Measurement Science and Technology, 2002. 13(9): p. 1425.

37. Robinson, J., T. Johnson, and L. Slater, Evaluation of known-boundary and resistivity constraints for improving cross-borehole DC electrical resistivity imaging of discrete fractures. Geophysics, 2013. 78(3): p. D115-D127.

38. Asfaw, Y. and A. Adler, Automatic detection of detached and erroneous electrodes in electrical impedance tomography. Physiological Measurement, 2005. 26(2): p. S175. 
39. Takada, A., Experimental study on propagation of liquid-filled crack in gelatin: Shape and velocity in hydrostatic stress condition. Journal of Geophysical Research, 1990. 95(B6): p. 8471-8481.

40. Robinson, J., et al., Strategies for characterization of fractured rock using crossborehole electrical tomography. The Leading Edge, 2013. 32(7): p. 784-790.

41. Herwanger, J., et al., A comparison of cross-hole electrical and seismic data in fractured rock. Geophysical prospecting, 2004. 52(2): p. 109-121.

42. Piersol, A., Time delay estimation using phase data. Acoustics, Speech and Signal Processing, IEEE Transactions on, 1981. 29(3): p. 471-477.

43. Ziola, S.M., Source location in thin plates using crosscorrelation. 1991, DTIC Document.

44. Liptai, R., et al., Acoustic emission techniques in materials research. International Journal of Nondestructive Testing, 1971. 3: p. 215-275. 


\section{Appendix A: Copyrighted material permission documentation}

\section{Permission for Figure 1-1}

Dear Evan:

Thank you for your request. John Wiley \& Sons, Inc. has no objections to your proposed reuse of this material.

Permission is hereby granted for the use requested subject to the usual acknowledgements (title, volume number, issue number, year, page numbers. Copyright [year and owner]. And the statement "This material is reproduced with permission of John Wiley \& Sons, Inc."). Any third party material is expressly excluded from this permission. If any of the material you wish to use appears within our work with credit to another source, authorization from that source must be obtained.

This permission does not include the right to grant others permission to photocopy or otherwise reproduce this material except for accessible versions made by non-profit organizations serving the blind, visually impaired and other persons with print disabilities.

Sincerely,

Paulette Goldweber

Associate Manager/Permissions-Global Rights

Professional Development 


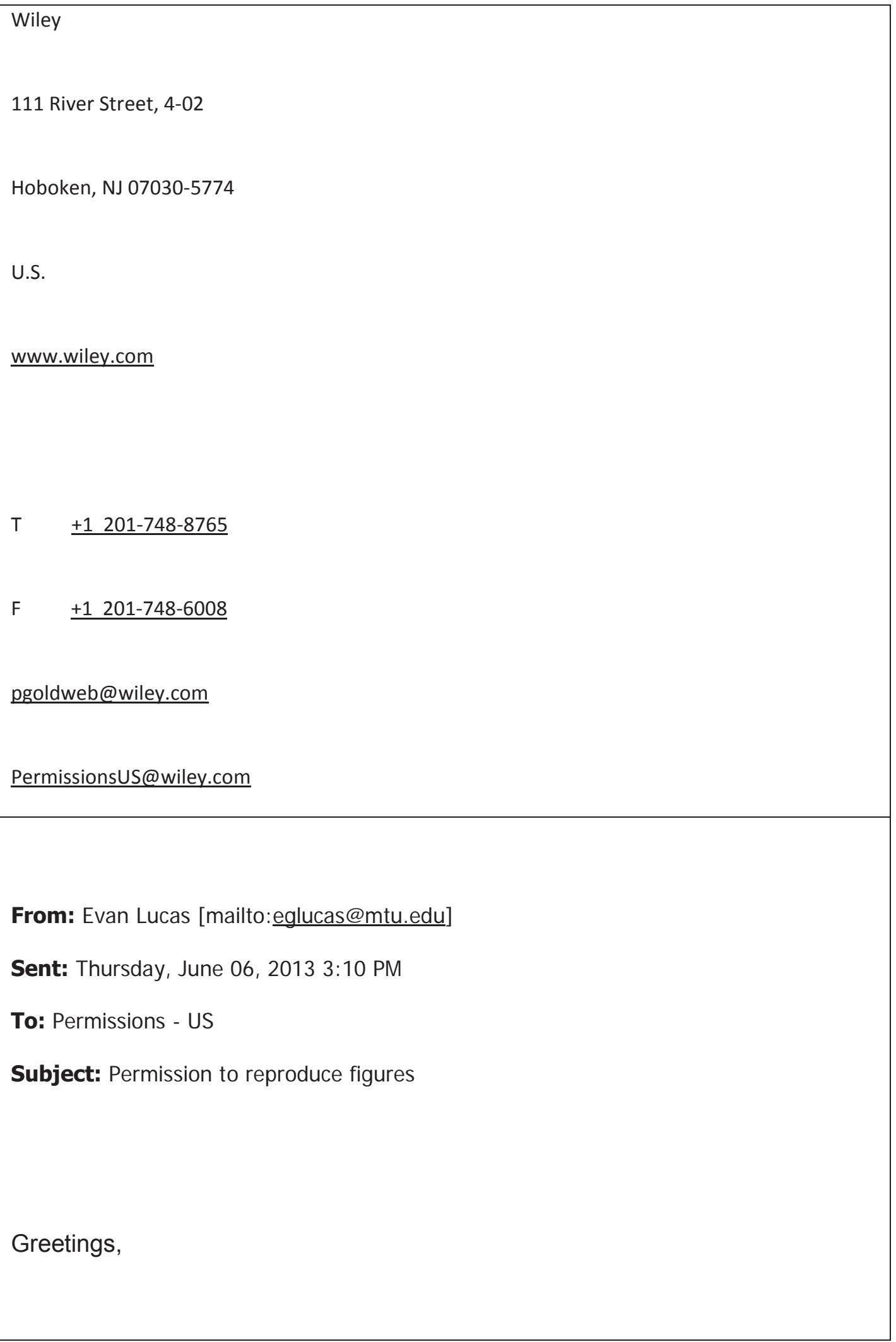




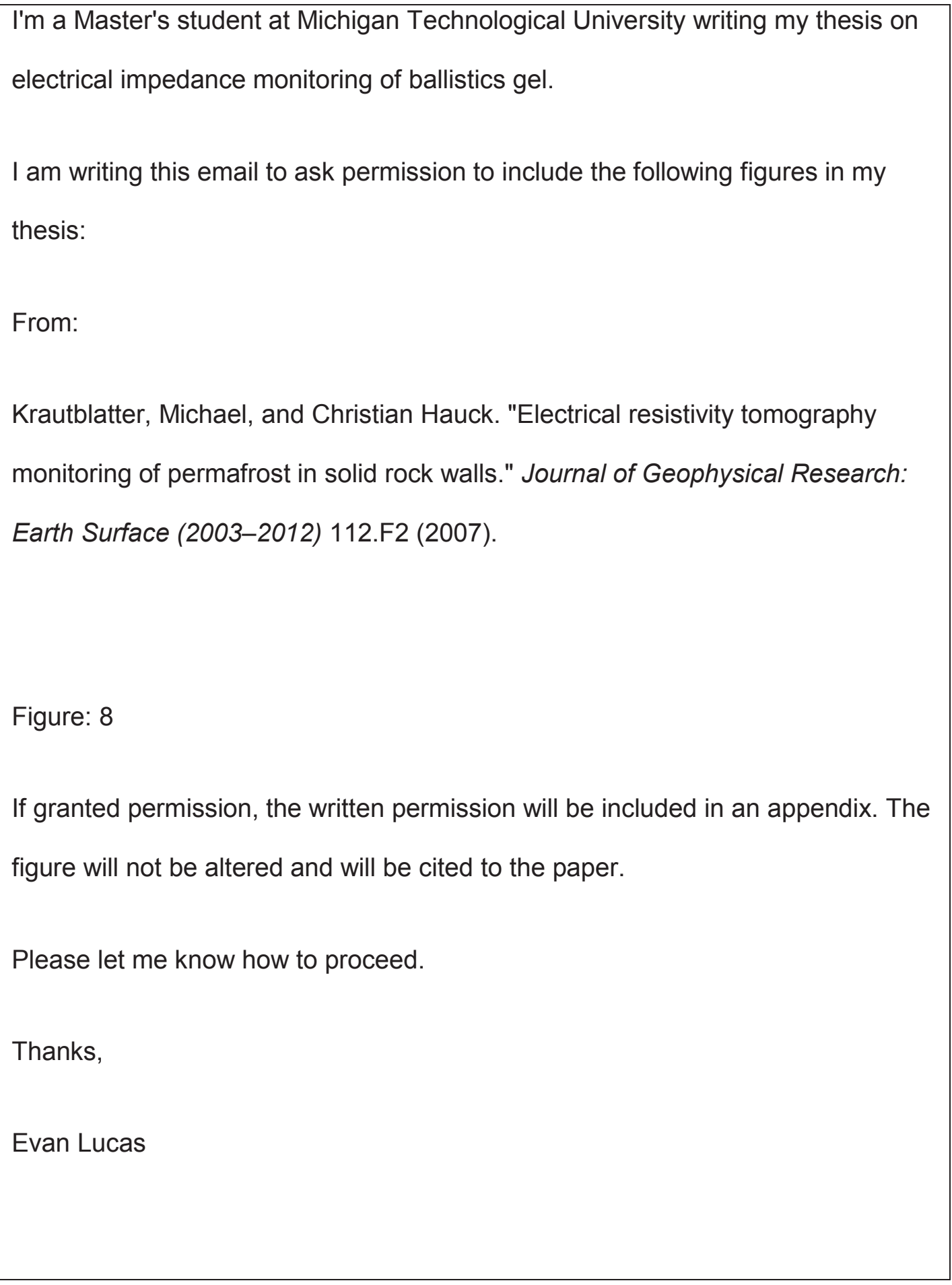




\section{Permission for Figure 1-2}

Evan,
The copyright rests with the publisher. If you have their approval then I am happy
for you to proceed, please give acknowledgement in the figure caption.
I applaud your diligence in pursuing this approval and good luck with the thesis.
Dr. York,
So: Trevor York
Trevor York.


I'm a Master's student at Michigan Technological University writing my thesis on electrical impedance monitoring of ballistics gel.

I am writing this email to ask permission to include the following figures in my thesis:

From:

Murphy, S. C., et al. "3D electrical tomographic imaging using vertical arrays of electrodes." Measurement Science and Technology 17.11 (2006): 3053.

Figures: 7

If granted permission, the written permission will be included in an appendix. The figure will not be altered and will be cited to the paper.

I have already contacted the publisher and if you grant me permission I will be able to use the figure.

Thanks,

Evan Lucas 


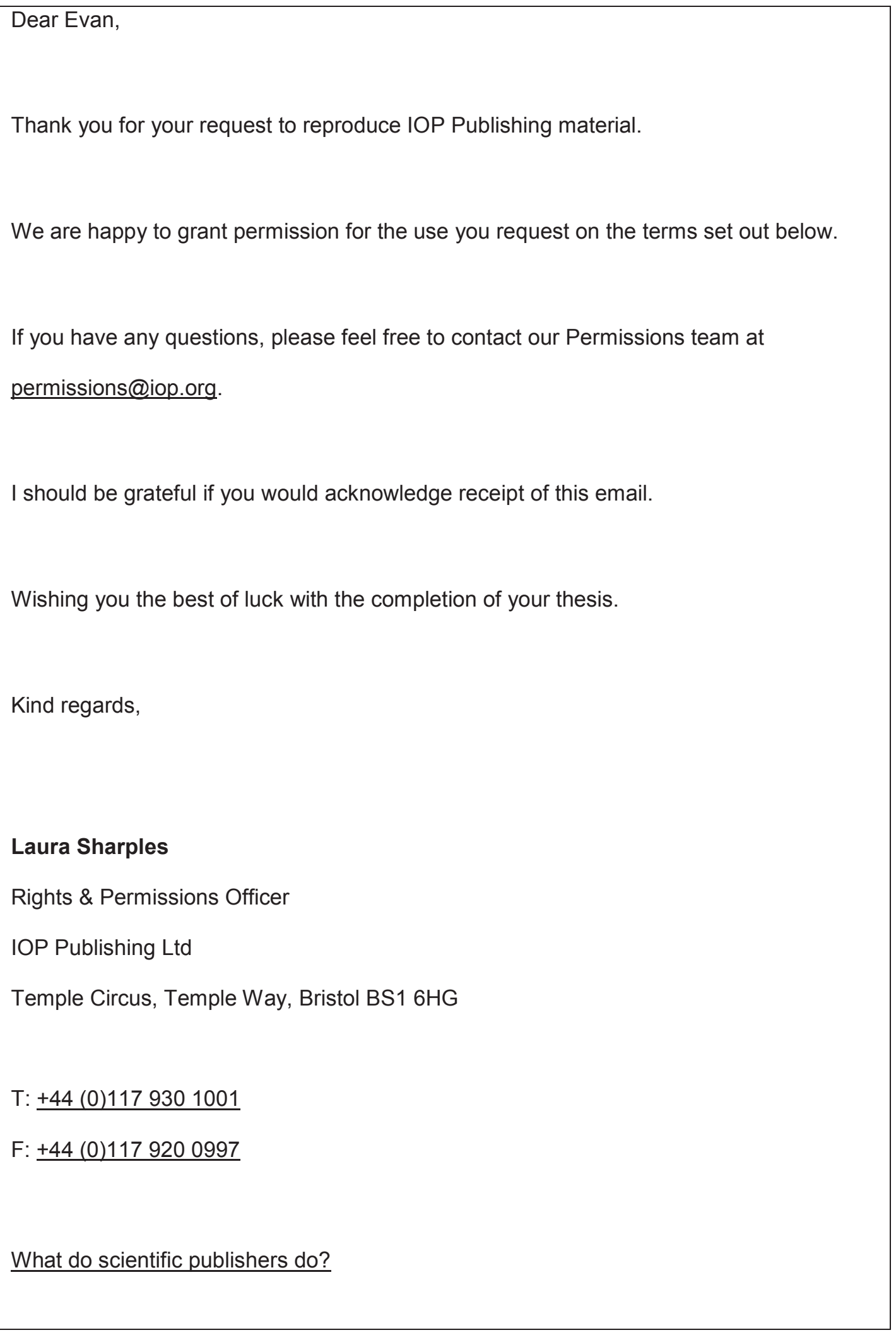




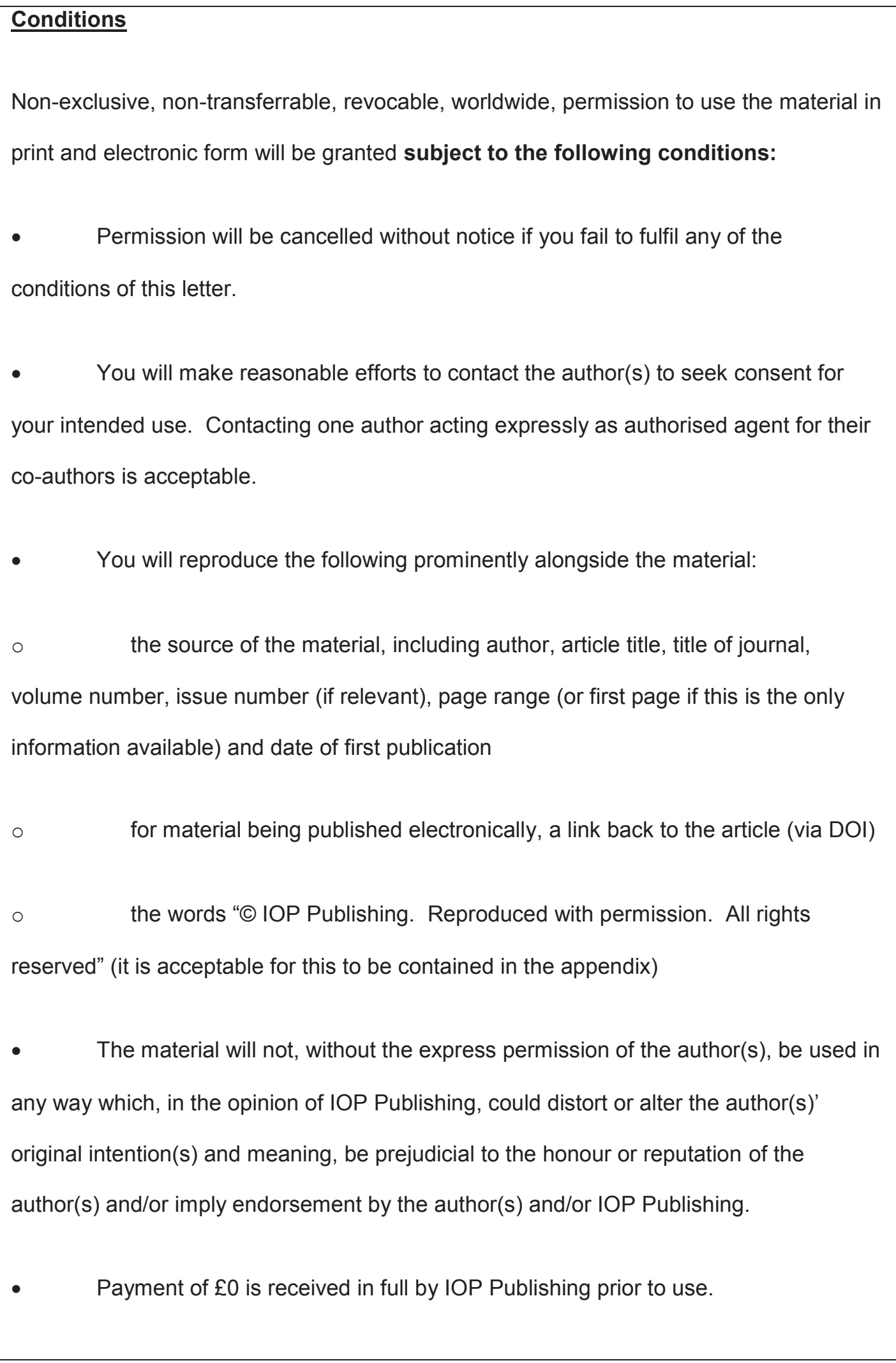


Please note: IOP does not usually provide signed permission forms as a separate attachment. Please print this email and provide it to your publisher as proof of permission.

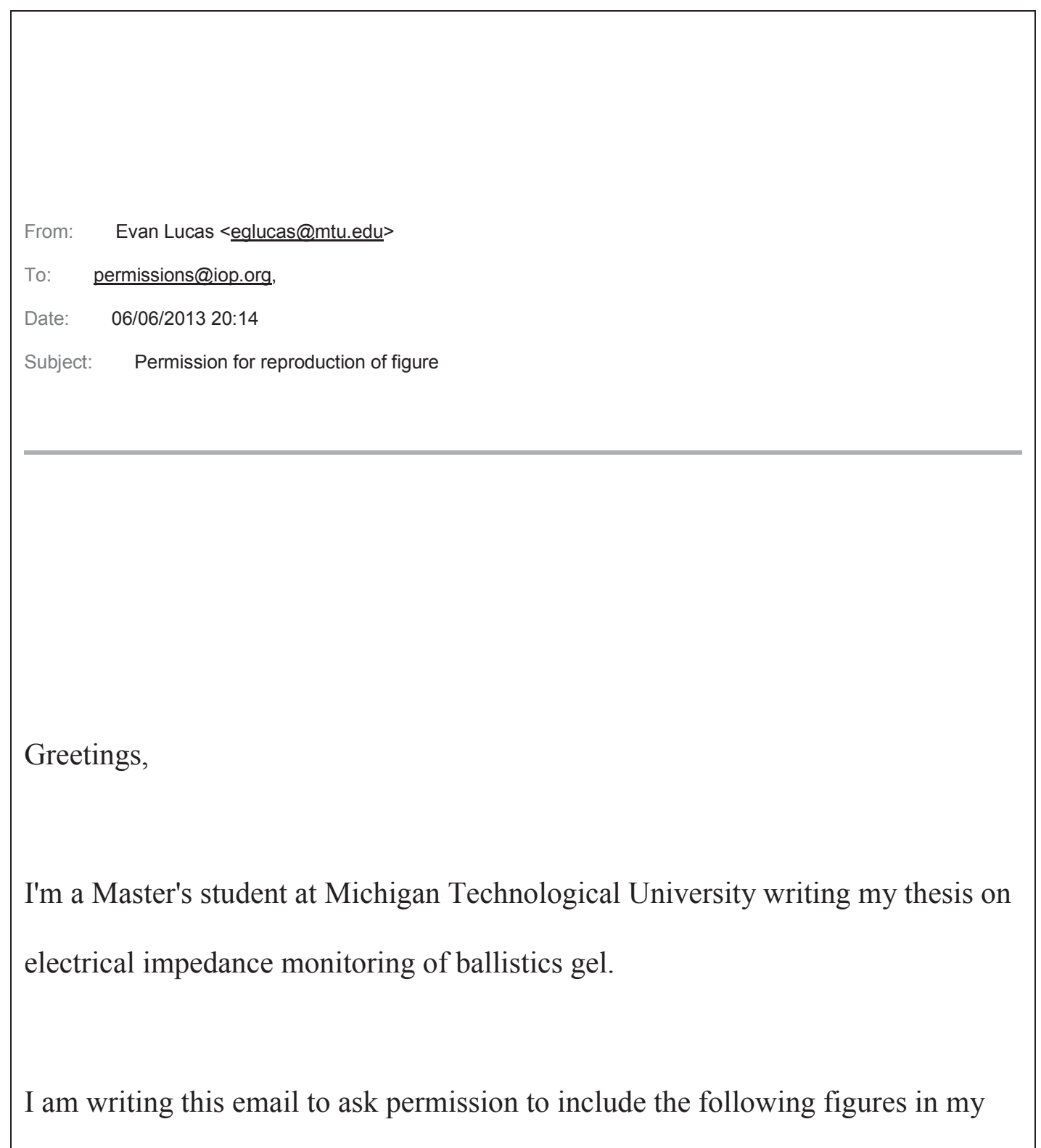




thesis:
From:
Murphy, S. C., et al. "3D electrical tomographic imaging using vertical arrays of
electrodes." Measurement Science and Technology 17.11 (2006): 3053 .
Figures: 7
Thanks,
If granted permission, the written permission will be included in an appendix. The
figure will not be altered and will be cited to the paper.




\section{Permission for Figure 1-3 and Figure 1-4}

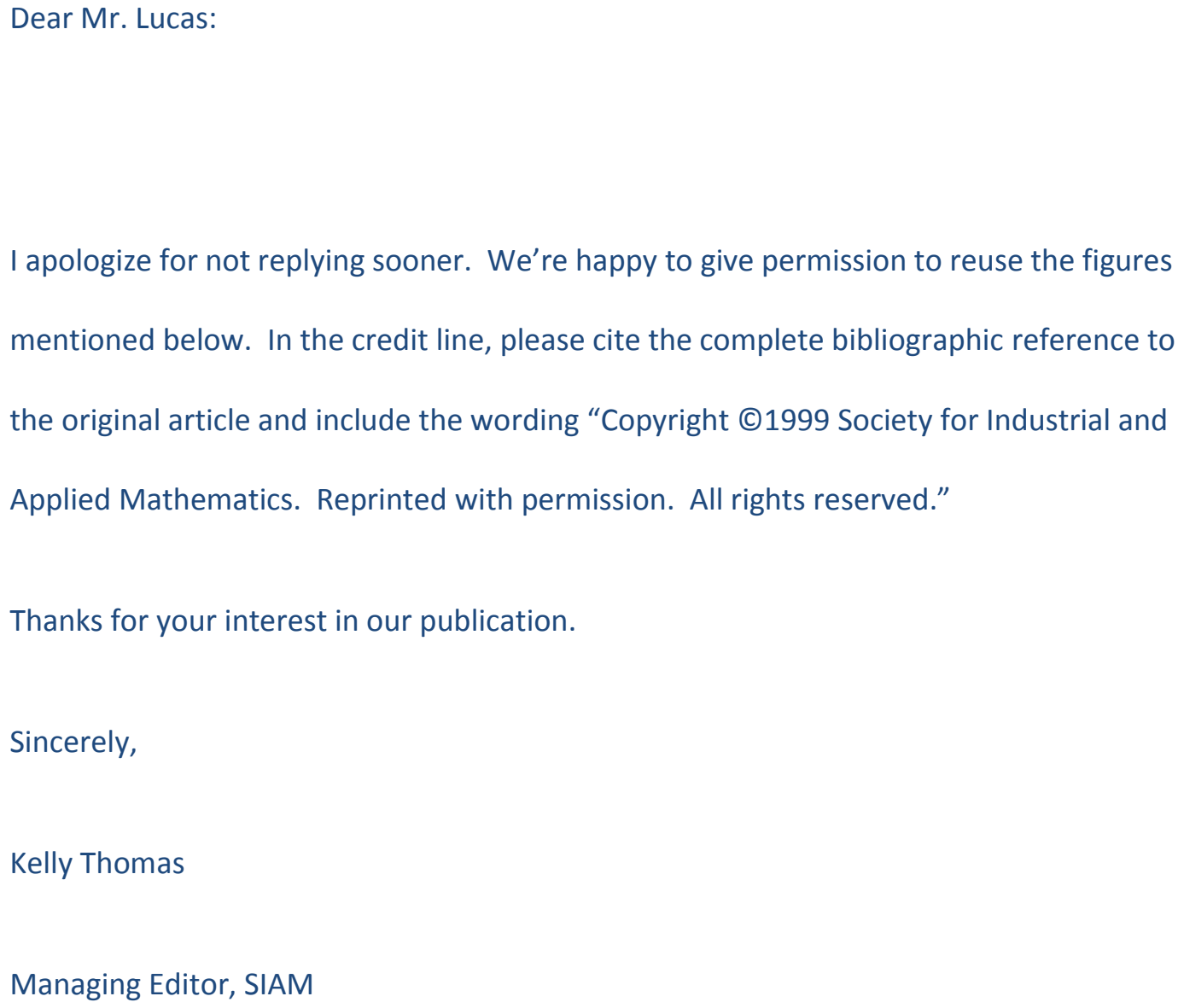

From: Evan Lucas [mailto:eglucas@mtu.edu]

Sent: Thursday, J une 06, 2013 3:07 PM

To: Kelly Thomas

Subject: Re: Permission for reproduction of image 


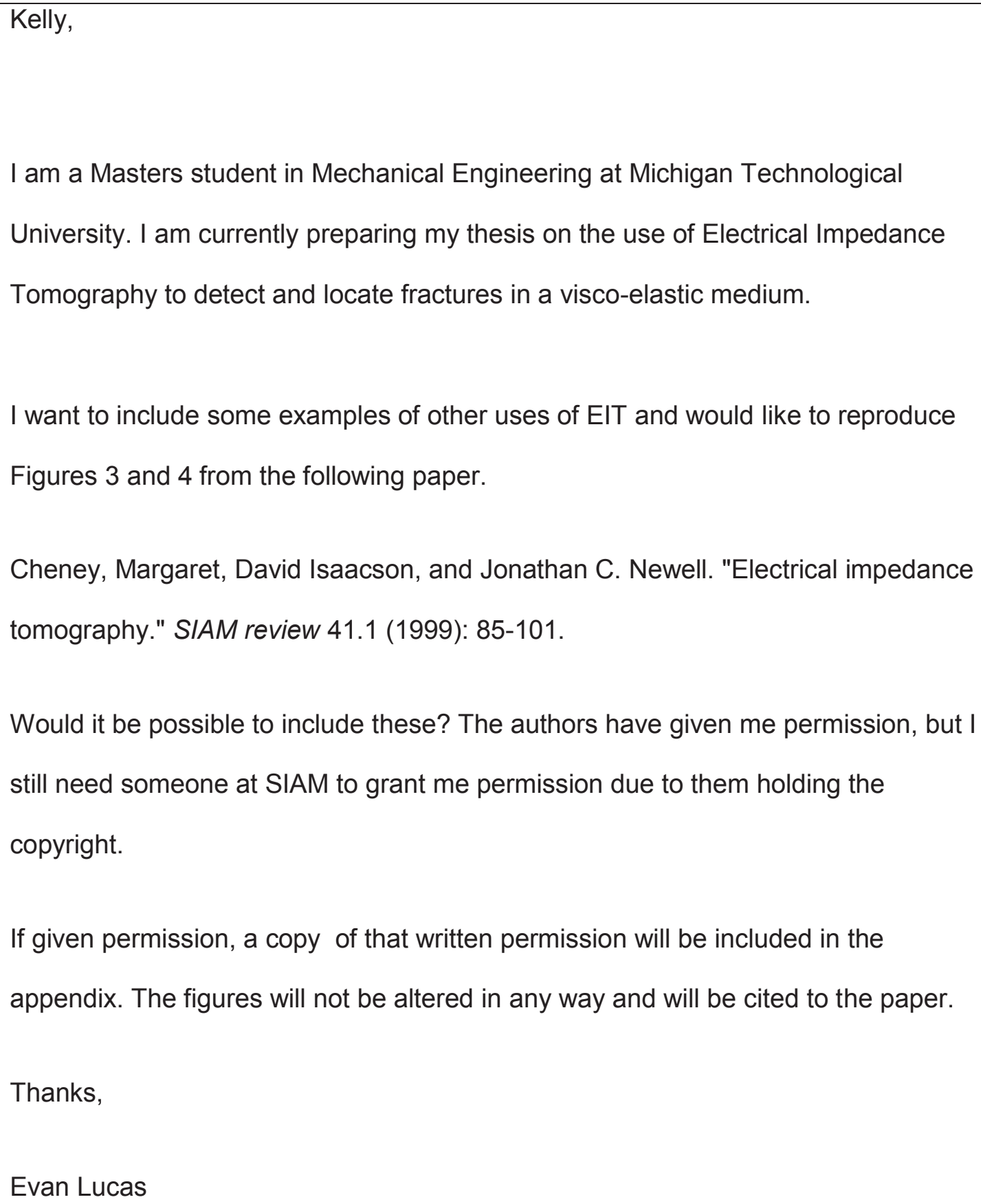


Dear Lucas,

Feel free to use what ever we have published. I would be interested in seeing your thesis some day if you would want to share it.

Good luck with your studies.

Respectfully,

David Isaacson

Sent from my iPad

On May 31, 2013, at 2:55 PM, Jonathan Newell <newelj@rpi.edu> wrote:

I second Dr. Cheney's response.

J. Newell

On 5/31/2013 12:59 PM, Margaret Cheney wrote:

Hi Evan,

Sure, it's fine with me, assuming you cite the paper. I'm copying my co-authors in case they have any issues. However I think the copyright is held by SIAM, so probably you should be asking someone listed under "journals" at http://siam.org/about/more/staff.php, where you can also find contact information. Maybe Mitch Chernoff (chernoff@siam.org) would be the right person?

Thanks for checking! Best wishes, Margaret Cheney 
On Fri, May 31, 2013 at 10:01 AM, Evan Lucas <eglucas@mtu.edu> wrote:

Dr. Cheney,

I am a Masters student in Mechanical Engineering at Michigan Technological

University. I am currently preparing my thesis on the use of Electrical Impedance

Tomography to detect and locate fractures in a visco-elastic medium.

I want to include some examples of other uses of EIT and would like to reproduce

Figures 3 and 4 from the following paper.

Cheney, Margaret, David Isaacson, and Jonathan C. Newell. "Electrical impedance tomography." SIAM review 41.1 (1999): 85-101.

Would it be possible to include those?

Thanks,

Evan Lucas 


\section{Permission for Figure 1-5}

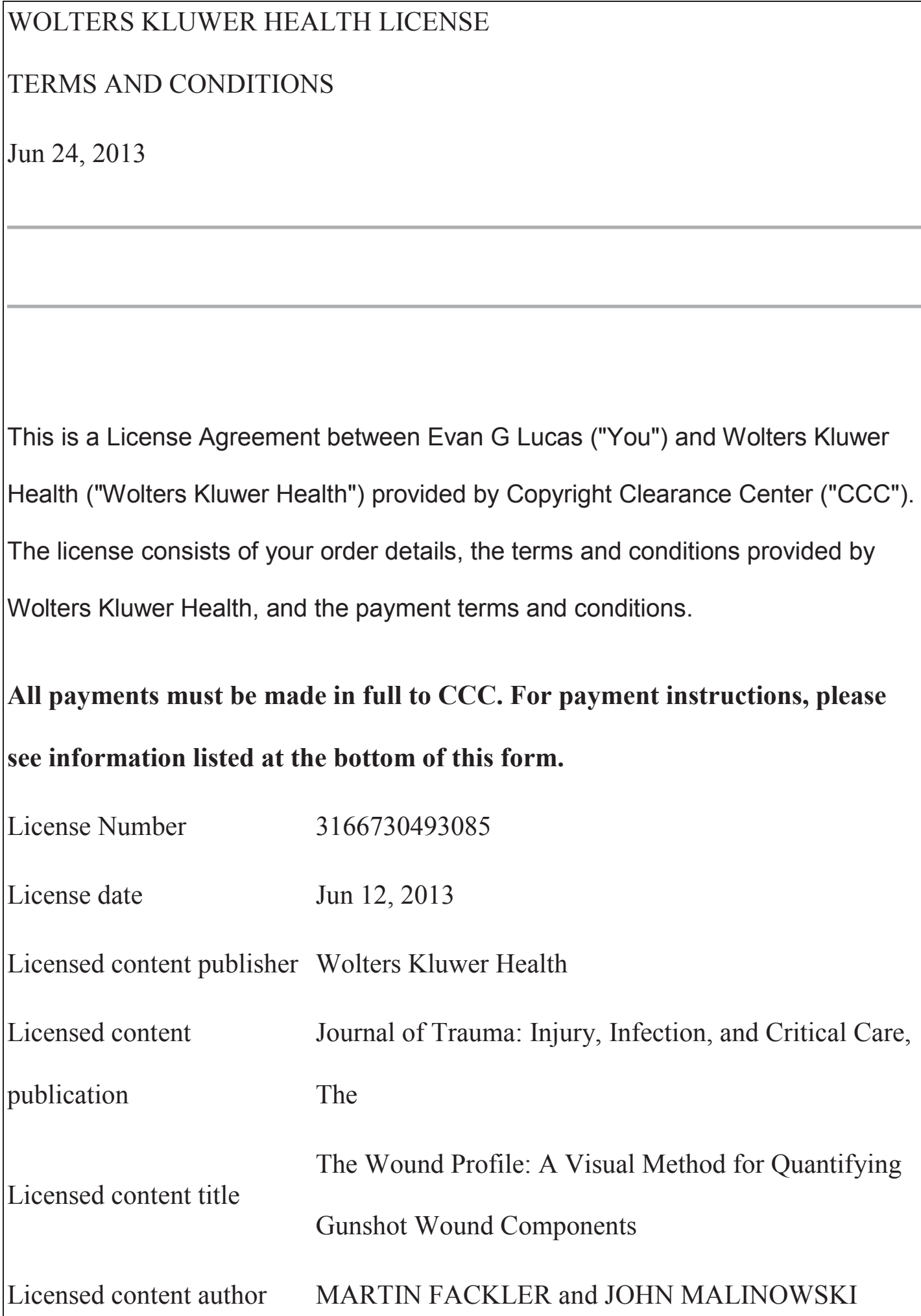




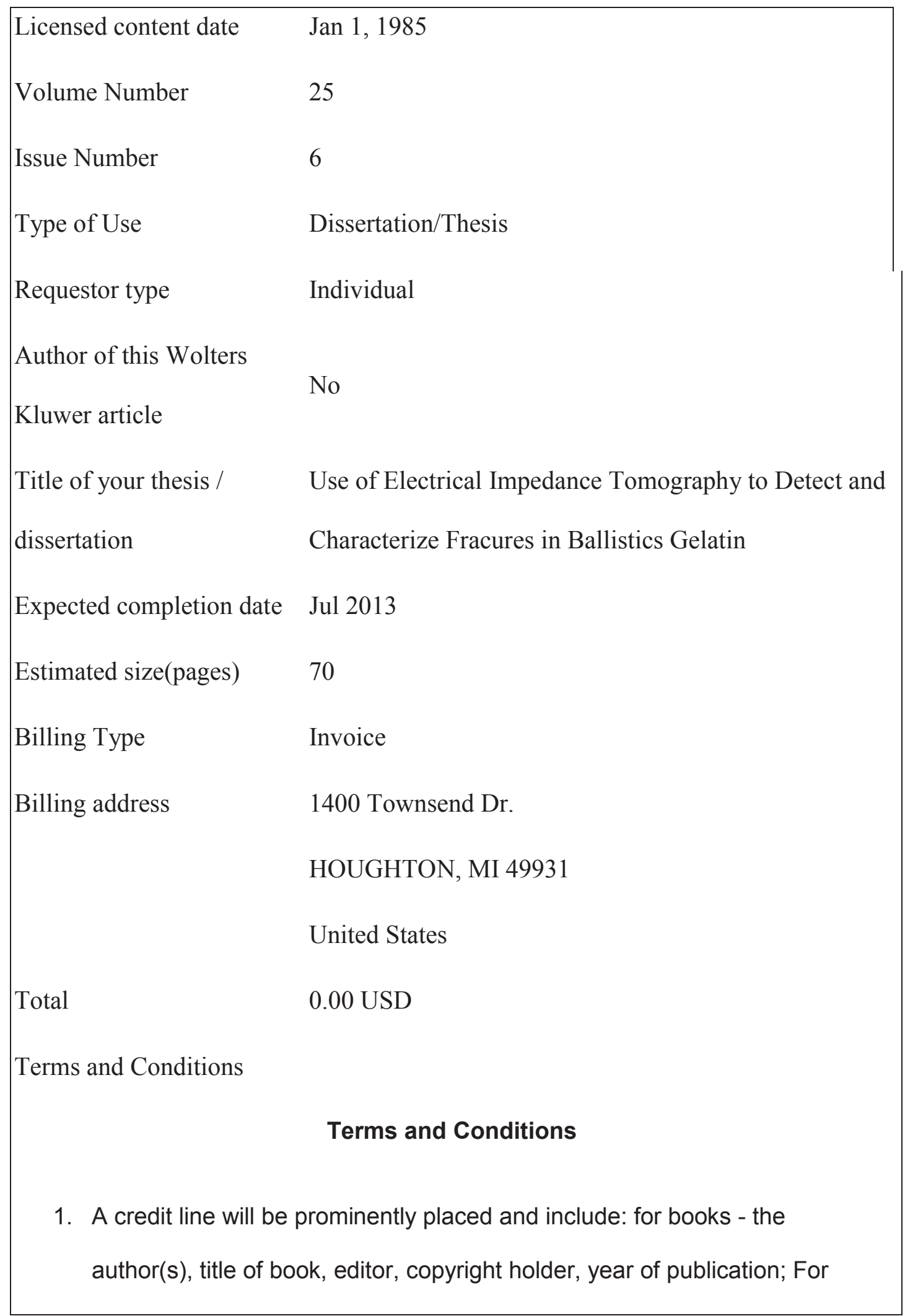


journals - the author(s), title of article, title of journal, volume number, issue number and inclusive pages.

2. The requestor warrants that the material shall not be used in any manner which may be considered derogatory to the title, content, or authors of the material, or to Wolters Kluwer.

3. Permission is granted for a one time use only within 12 months from the date of this invoice. Rights herein do not apply to future reproductions, editions, revisions, or other derivative works. Once the 12-month term has expired, permission to renew must be submitted in writing.

4. Permission granted is non-exclusive, and is valid throughout the world in the English language and the languages specified in your original request.

5. Wolters Kluwer cannot supply the requestor with the original artwork or a "clean copy."

6. The requestor agrees to secure written permission from the author (for book material only).

7. Permission is valid if the borrowed material is original to a Wolters Kluwer imprint (Lippincott-Raven Publishers, Williams \& Wilkins, Lea \& Febiger, Harwal, Igaku-Shoin, Rapid Science, Little Brown \& Company, Harper \& Row Medical, American Journal of Nursing Co, and Urban \& Schwarzenberg - English Language).

8. If you opt not to use the material requested above, please notify Rightslink within 90 days of the original invoice date.

9. Please note that articles in the ahead-of-print stage of publication can be cited and the content may be re-used by including the date of access and 
the unique DOI number. Any final changes in manuscripts will be made at the time of print publication and will be reflected in the final electronic version of the issue.

Disclaimer: Articles appearing in the Published Ahead-of-Print section have been peer-reviewed and accepted for publication in the relevant journal and posted online before print publication. Articles appearing as publish aheadof-print may contain statements, opinions, and information that have errors in facts, figures, or interpretation. Accordingly, Lippincott Williams \& Wilkins, the editors and authors and their respective employees are not responsible or liable for the use of any such inaccurate or misleading data, opinion or information contained in the articles in this section.

10. This permission does not apply to images that are credited to publications other than Wolters Kluwer journals. For images credited to non-Wolters Kluwer journal publications, you will need to obtain permission from the journal referenced in the figure or table legend or credit line before making any use of the image(s) or table(s).

11. The following statement needs to be added when reprinting the material in Open Access publications: 'promotional and commercial use of the material in print, digital or mobile device format is prohibited without the permission from the publisher Lippincott Williams \& Wilkins. Please contact journalpermissions@lww.com for further information.

12. In case of Disease Colon Rectum, Plastic Reconstructive Surgery, The Green Journal, Critical Care Medicine, Pediatric Critical Care Medicine, the American Heart Publications, the American Academy of Neurology 


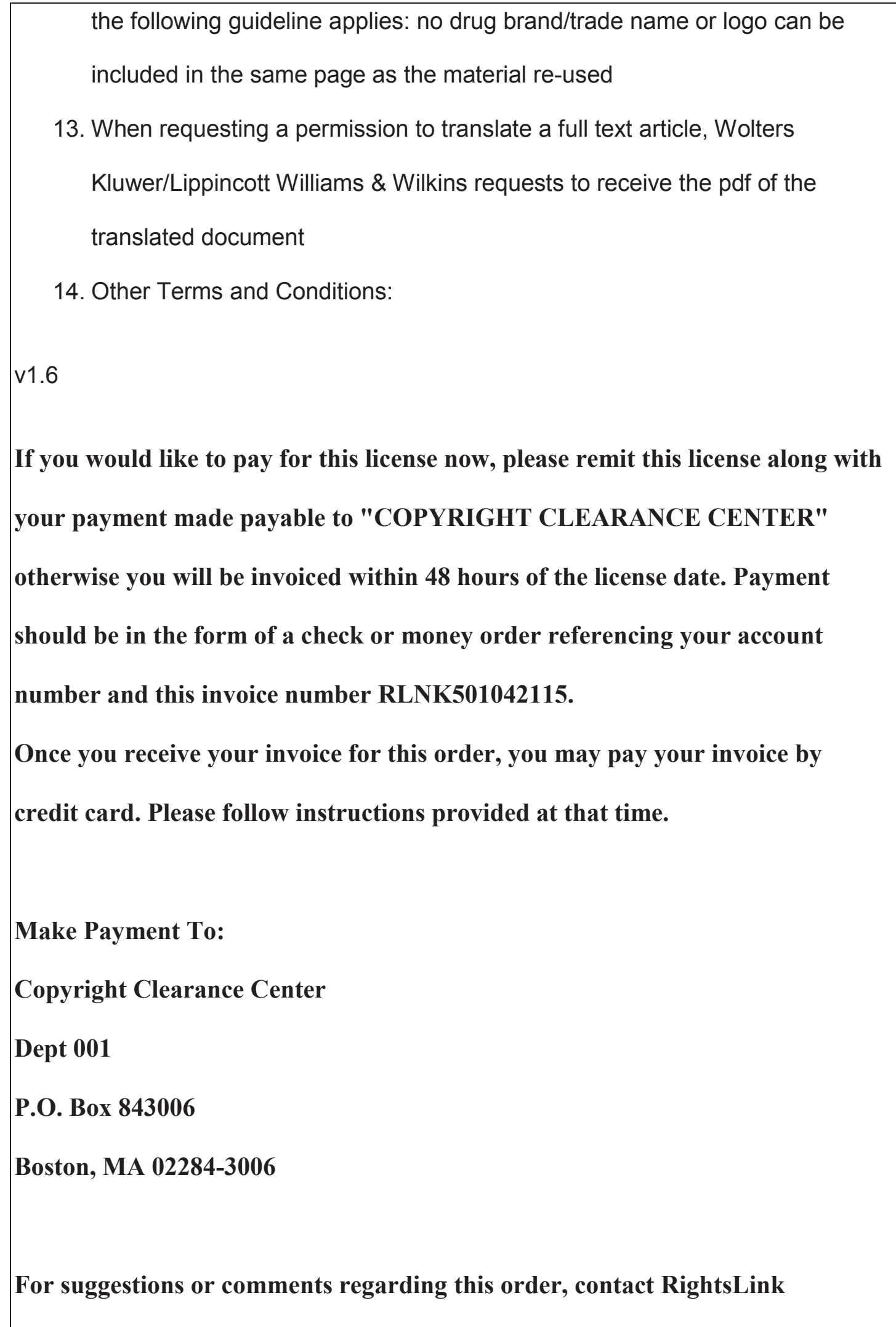


Customer Support: customercare@copyright.com or +1-877-622-5543 (toll free in the US) or +1-978-646-2777.

Gratis licenses (referencing $\$ 0$ in the Total field) are free. Please retain this printable license for your reference. No payment is required. 


\section{Appendix B: Previous work}

The initial attempts to solve the problem of detecting fractures in a visco-elastic medium during dynamic events followed more traditional Structural Health Monitoring (SHM) methods utilizing wave propagation techniques. Due to the damping properties of the visco-elastic materials being used, detecting fractures using these methods that depend were not very successful.

The first methods proposed and tested involved acoustic methods. This can be easily understood by thinking of a source-path-receiver system. By considering the defect's scattered energy as the source and measuring the response, the source location can be estimated if there is some a priori knowledge of the path. The prior knowledge in this case is the speed of sound in the material. To accurately measure velocity, two accelerometers were mounted on either end of the sample and one end was excited. Both hammers and shakers were tested in the role of the excitation source. Time delay was estimated in two ways - from phase information [42] and from cross correlating. [43]

The next proposed method was to employ source-receiver SHM techniques. This would involve having a source excite the structure with a known waveform. Receivers attached to the structure would use the measured response to detect and locate damage to the system. There is a large amount of research existing in this area, generally using piezoelectric sources and receivers. A proof of concept test was developed using a fishfinder to look for discontinuities in the visco-elastic system. This test ended in a complete failure for reasons unknown at the time.

Following the same concept, a small piezoelectric shaker designed for active vibration control was used as a source. Inspired by standard seismic acquisition and active radar 
techniques, a frequency modulated input (linear chirp) was used. This allowed separation of direct and reflected arrivals by cross-correlating with the input signal. While varying the frequency content of the chirp inputs, it was found that higher frequencies were greatly attenuated. This was confirmed by taking coherence measurements between a load cell at the source and an accelerometer at the opposite end of the structure. This helped explain the issues experienced with the fish-finder, as the fishfinder operated in a high frequency range where great attenuation was experienced.

Consideration was also given to a fully passive SHM method, using transmissibility functions between receivers on the structure to detect and locate fractures and other damage to the structure. This was based on the work of Johnson. [25]This was significantly more successful, giving us a reliable indicator that damage had occurred. Unfortunately, magnitude and location of the damage that occurred were not successfully determined using this method.

Another proposed method in this area was exploiting the acoustic emission (AE) that occurs during material failure to provide information on the failure mode, magnitude, and position. During plastic deformation and material failure, there are two primary modes of AE grain boundary slip and grain resonance. Although the boundary slip is a broad spectrum emission, the grain resonance is a predictable narrow band event. The grain resonance is excited more strongly in high strength brittle materials, which indicates that the $A E$ in a viscoelastic system will be smaller. This method is commonly used in many fields of study, including mining, gas and oil, and composites SHM. Tension testing on visco-elastic samples yielded characteristic AE signatures. However, due to the same attenuation issues previously encountered, this method was also abandoned. [44] 